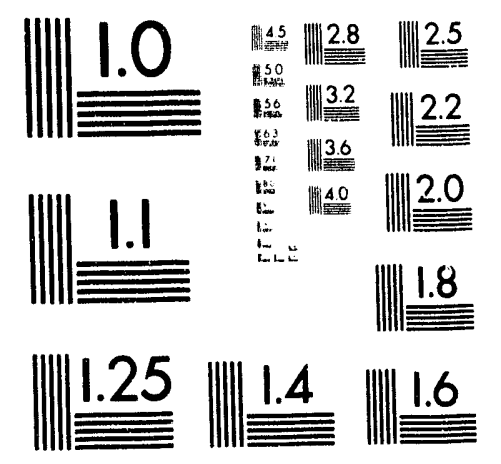



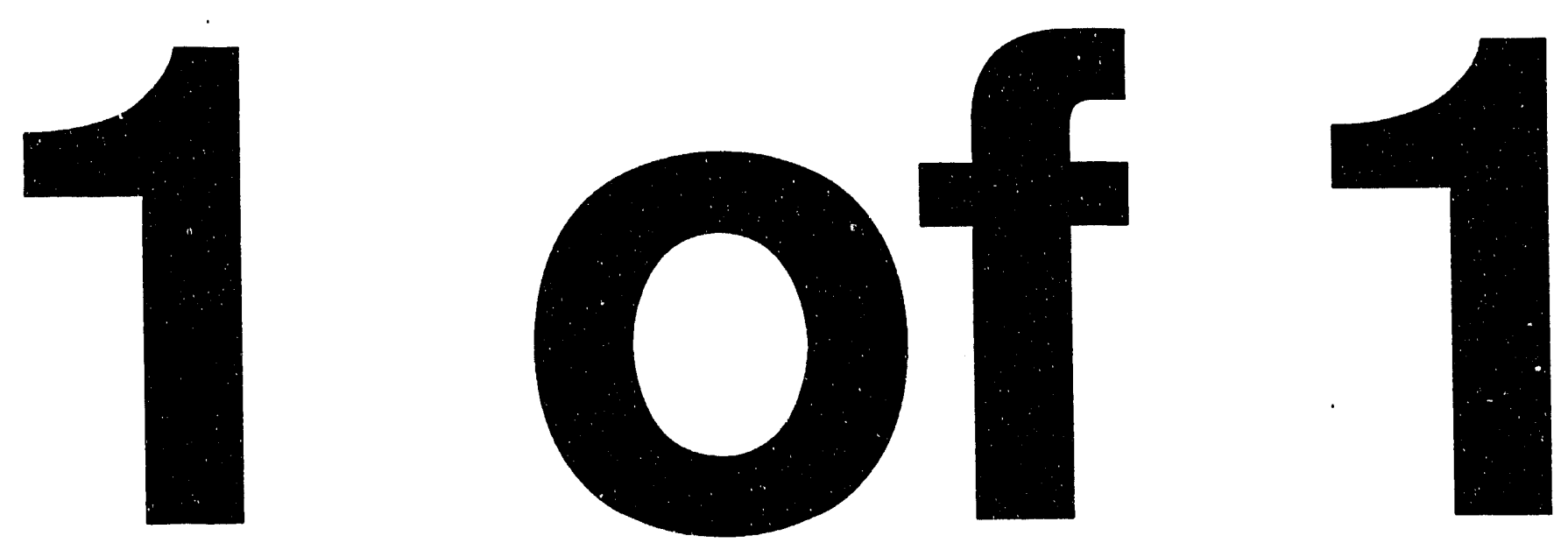

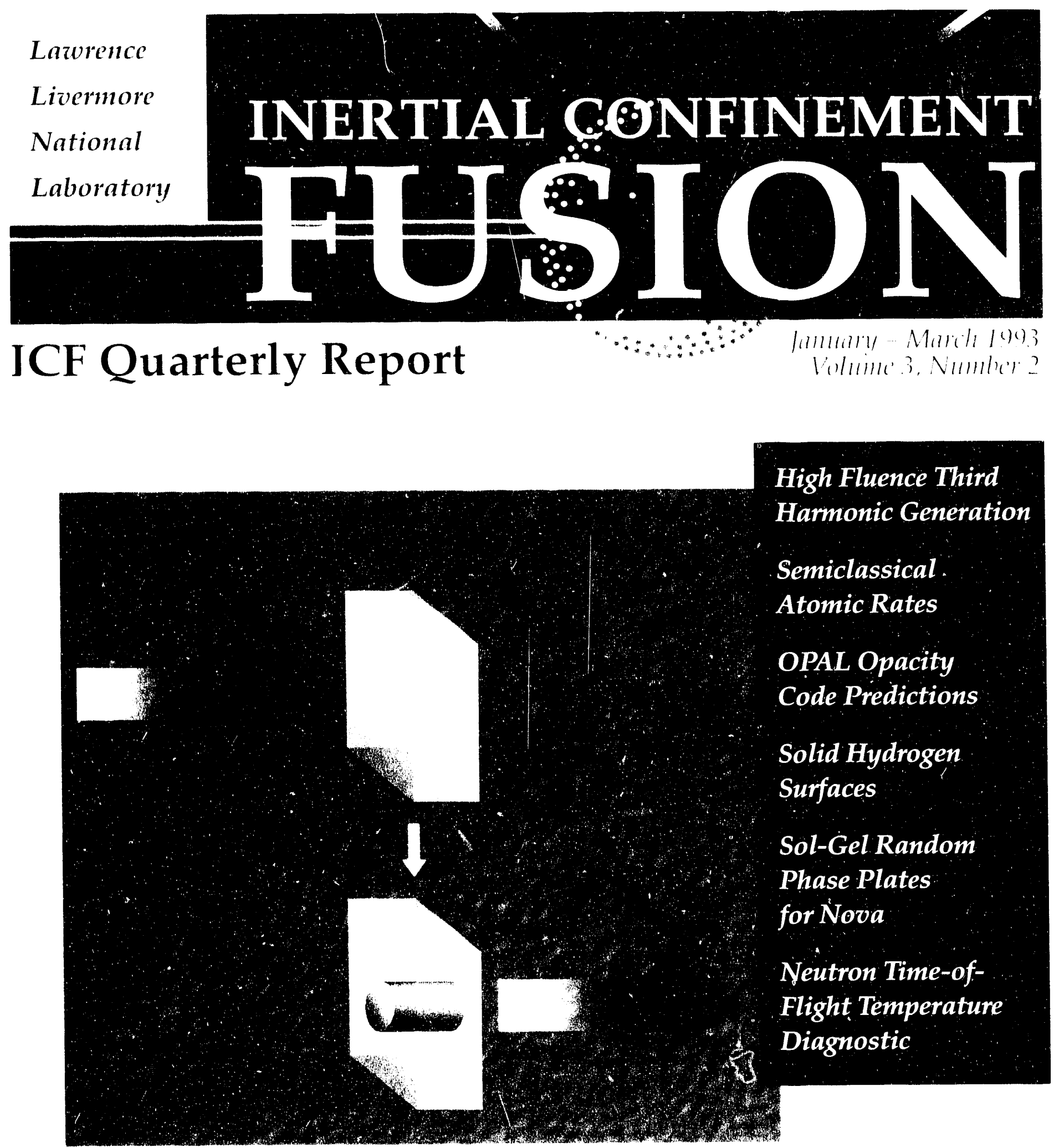

Ulltraziolet Induced

Transicnt Absorption

in $K D P$ 
The ICF Quarterly Report is published four times each fiscal year by the Inertial Confinement Fusion Program at the Lawrence Livermore National Laboratory. The journal reports selected current research within the ICF Program. Major areas of investigation presented here include fusion target theory and design, target fabrication, target experiments, and laser and optical science and technology. Questions and comments relating to the technical content of the journal should be addressed to the ICF Program Office, Lawrence Livermore National Laboratory, P.O. Box 5508, Livermore, CA 94551.

The Cover: Schematic of the transient-absorption process in KDP. Two-photon absorption of a deep ultraviolet beam (top) can produce broadband absorbing defect states in normally transparent KDP crystals that remain for several days, leaving a gray track (bottom) that is even visible to the eye. These states may, in turn, absorb a substantial fraction of a subsequent light pulse. Transient absorption can substantially reduce the frequency-conversion efficiency in KDP of the fourth harmonic of a Nd:glass laser system. However, calculations at the third harmonic predict a conversion efficiency reduction of less than one percent. See the article, "Ultraviolet Induced Transient Absorption in KDP and Its Influence on Fourth Harmonic Frequency

Conversion," page 69.
UCRL-LR-105821-93-2

Distribution Category UC-712 January - March 1993
Printed in the United States of America Available from

National Technical Information Service

U.S. Department of Commerce 5285 Port Royal Road

Springfield, Virginia 22161

Price codes: printed copy A03, microfiche A01.
This document was prepared as an account of work sponsored by an agency of the United States Government. Neither the United States Government nor the University of California nor any of their employees makes any warranty, express or implied, or assumes any legal liability or responsibility for the accuracy, completeness, or usefulness of any information, apparatus, product, or process disclosed, or represents that its use would not infringe privately owned rights.

Reference herein to any specific commercial products, process, or service by trade name, trademark, manufacturer, or otherwise, does not necessarily constitute or imply its endorsement, recommendation, or favoring by the United States Government or the University of California. The views and opinions of authors expressed herein do not necessarily state or reflect those of the United States Government or the University of California and shall not be used for advertising or product endorsement purposes.

Work performed under the auspices of the U.S. Department of Energy by Lawrence Livermore National Laborator, under Contract W-7405-Eng-48. 


\section{INERTIAL CONFINEMENT FUSION \\ Jamuary - March 1993 \\ Volume 3, Number 2}

\section{Quarterly Report}

\section{In this issue:}

Foreword

High Fluence Third Harmonic Generation

Designs for the next generation of Nd:glass-based fusion laser will emplov beam propagation and harmenic generation at much higher fluences than have been possible in the past. We describe the first of a series of harmonic generation experiments performed on the Optical Sciences Laser in support of the high fluence harmonic generation subsystem for the Beamlet Demonstration Project laser.

Ultraviolet Induced Transient Absorption in KDP and Its Influence on Fourth Harmonic Frequency Conversion

This article discusses deep ultraviolet irradiation of KDP crystals. The resulting transient absorption can substantially affect the fourth harmonic frequency-conversion efficiency of a Nd:glass laser system.

Relativistic Semiclassical Atomic Transition Rates

We developed a relativistic semiclassical calculation of radiative rates based on a new WKB solution of the Dirac equation for an electron in a spherically symmetric atomic potential $L(r)$. The wave function is simple, accurate, and compatible with previous work.

\section{Verification of OPAL Opacity Code Predictions} for Conditions of Astrophysical Interest

Recent advances in experiments with laser-produced plasmas permit measurements of photon absorption for conditions relevani to stellar envelopes, thus providing direct tests of opacity models.

\section{Solid Hydrogen Surfaces}

Our series of D-T beta layer experiments shows a surface roughness amplitude larger than that required for an NIF. In nontritiated hydrogen laver experiments, we observed significant, continued improvements in the surface finish.

\section{Large Aperture Sol-Gel Random Phase Plates}

\section{for Beam Snoothing on Nova}

We developed a novel process for fabricating s(o)-gel random phase plates (RPPs). The procedure can easily be scaled to large aperture substrates and yields high quality products with excellent optical performance and high optical damage thresholds. We have fabricated several bi-level RPPs of different aperture and element sizes for use in infrared, visible, and ultraviolet wavelengths.

Scientific Editor Peter A. Amendt

\section{Editorial Staff}

Marie Kotowski

Peter W. Murphy

Design Staff

Ellen L. Baldwin

Art Staff

TID Art Division

\section{Neutron Time-of-Flight Ion Temperature Diagnostic for Nova}

We have constructed and installed an ion temperature diagnostic for indirect-drive Nova targets, whose neutron yields are typically too low to measure with current-mode time-of-flight detectors.

Facilities Report, January - March 1993

\section{Publications}

\section{MASTER}

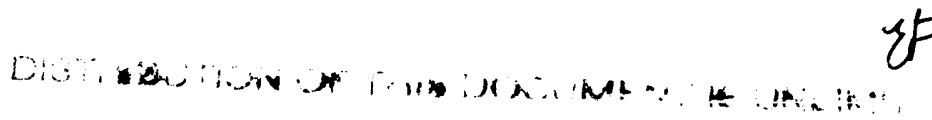




\section{FOREWORD}

This issue of the ICF Quarterly contains seven articles describing recent advances in Lawrence Livermore National Laboratory's inertial confinement fusion (ICF) program. The current emphasis of the ICF program is to design a 1-2 MJ glass laser for the proposed National Ignition Facility (NIF). The enclosed articles describe recent Nova experiments and theoretical investigations tailored towards enhancing our understanding of the key physics issues for the NIF.

Three of the articles are devoted to improved laser beam quality and efficient harmonic generation. The article entitled "Smoothing on Nova" describes the use of sol-gel random phase plates for high optical damage threshold beam smoothing. Beam smoothing is important in controlling and possibly suppressing the various hydrodynamic and plasma instabilities that can thwart a symmetric implosion. "High Fluence Third Harmonic Generation" provides a summary of recent progress in obtaining high fluence harmonic generation appropriate for the next generation Nd:glass based laser. The article "Ultraviolet Induced Transient Absorption in KDP' and Its Influence on Fourth Harmonic Frequency Conversion" explores the implications of transient absorption on efficient fourth harmonic frequency conversion. The understanding of the underlying physical process should enable designers of frequency conversion systems to minimize the effects of transient absorption and maximize the final output $4(1)$ energy for efficient laser-target coupling.

Two articles deal with radiative processes in laser heated plasmas and relevant aspects of our current modeling capability. In "Relativistic Semiclassical Atomic Transistion Rates," we discover how the use of a new WKB solution of the Dirac equation enhances our understanding of atomic processes in high- $Z$ materials for the high-temperature environment characteristic of the NIF. "Verification of OPAL Opacity Code Predictions for Conditions of Ass nphy":cal Interest" describes some recent advances in experimental tests of our opacity modeling. Both articles represent important advances in our modeling of the NIF regime plasmas; this capability will depend heavily on the simulation code LASNEX.

NIF targets will require thick layers of hydrogen ice inside a several millimeter diameter shell to reach the conditions necessary for ignition. The article "Solid Hydrogen Surfaces" reports on progressive improvements in the surface finish using nontritiated hydrogen layering.

Collaboration between Los Alamos National Laboratory, Lawrence Livermore National Laboratory, and EG\&G has resulted in the construction of a new ion temperature diagnostic. As described in "Neutron Time-of-Flight Ion Temperature Detector," this tool measures neutron arrival time using an array of scintillator photomultiplier detectors and is designed for indirect-drive Nova targets, whose neutron yields are typically too low for accurate measurement with current time-of-flight detectors.

In summary, these seven articles demonstrate the depth and vitality of ICF's research effort in confronting key issues of the proposed NIF.

Peter A. Amendt

Scientific Editor 


\title{
High Fluence Third Harmonic Generation
}

\author{
C. E. Barker \\ D. Milam \\ R. Boyd
}

\section{Introduction}

Because the efficiency with which laser energy couples to ICF targets increases with decreasing laser wavelength, ${ }^{1}$ current and proposed $\mathrm{Nd}$ :glass-based laser systems for ICF experiments use optical harmonic generation techniques to produce intense visible and ultraviolet optical pulses. The optical harmonic generation system for the Nova laser consists of carefully coordinated arrays of KDP (potassium dihydrogen phosphate) crystals. ${ }^{2}$ To avoid damage to both the dielectric-coated transport mirrors and the KDP crystals on Nova, the laser beams exiting the final $46 \mathrm{~cm}$ amplifiers are expanded in the final spatial filters. This beam expansion reduces the average infrared fluence incident upon the transport mirrors and KDP crystals to $3 \mathrm{~J} / \mathrm{cm}^{2}$ or less for $3-\mathrm{ns}$ square pulses. The concept of a high-precision array of subaperture-sized harmonic generation crystals was thus motivated by the available size of high quality KDP plates. Very recently, improvements in KDP crystal growth have brought about a significant improvement in KDP damage thresholds. ${ }^{3}$ Similar improvements have also been realized in dielectric coatings. ${ }^{4}$ Taking advantage of these improvements in damage threshold, designs for the next generation of $\mathrm{Nd}$ :glass-based fusion lasers employ beam propagation and harmonic generation at much higher fluences than has been possible in the past. The Bearnlet Demonstration Project $^{5}$ laser design, for example, propagates average infrared fluences of up to $12 \mathrm{~J} / \mathrm{cm}^{2}$ in a 3-ns square pulse to the KDP harmonic generation crystals. In this article, we describe the first of a series of harmonic generation experiments performed on the Optical Sciences Laser $(\mathrm{OSL})^{6}$ in support of the high fluence harmonic generation subsystem for the Beamlet laser.

\section{Harmonic Generation with Plane Waves}

Optical harmonic generation is most often implemented on large and small laser systems through the use of sum-frequency mixing in birefringent crystals that possess a second-order nonlinear optical susceptibility. A simple yet general physical model of the sumfrequency mixing process is the interaction of two input plane waves, at frequencies $\omega_{1}$ and $\omega_{2}$, in a nonlinear medium to produce a third wave at the sum frequency, $\omega_{3}=\omega_{1}+\omega_{2}$, via the second-order nonlinear polarization. ${ }^{7}$ This process is described mathematically by three coupled, first-order, nonlinear differential equations that are obtained from Maxwell's equations by use of the slowly varying envelope approximation. ${ }^{8}$ For monochromatic plane waves propagating in the $\mathrm{z}$ direction, the electric fields are of the form

$$
\operatorname{Re}\left[E_{j} \exp \left(i \omega_{j} t-i k_{j} z\right)\right]
$$

with $j=1,2,3$, and the magnitude of the wave vector $k_{j}$ is given by

$$
k_{j}=\frac{\omega_{j} n_{j}\left(\omega_{j}\right)}{c},
$$

where $c$ is the speed of light in vacuum, $\omega_{j}$ is the angular frequency, and $n_{j}\left(\omega_{j}\right)$ is the refractive index seen by the $j$ th wave at frequency $\omega_{j}$. The relationship between the refractive index of a given material and the frequency (or wavelength) of light incident upon it is usually measured at a number of discrete wavelengths; this data is then fit to an analytical function known as a Sellmeier 
relation. ${ }^{9}$ Using monochromatic plane waves to describe the optical fields, the set of first-order coupled differential equations that describe sum-frequency mixing can be written as ${ }^{10}$

$$
\begin{aligned}
& \frac{d E_{1}^{\prime}}{d z}=\frac{-\gamma_{1}}{2} E_{1}^{\prime}-i K E_{3}^{\prime} E_{2}^{\prime \prime} \exp (-i \Delta k \cdot z) \\
& \frac{d E_{2}^{\prime}}{d z}=\frac{-\gamma_{2}}{2} E_{2}^{\prime}-i \frac{\omega_{2}}{\omega_{1}} K E_{3}^{\prime} E_{1}^{\prime \prime} \exp (-i \Delta k \cdot z) \\
& \frac{d E_{3}^{\prime}}{d z}=\frac{-\gamma_{3}}{2} E_{3}^{\prime}-i \frac{()_{3}}{\omega_{1}} K E_{1}^{\prime} E_{2}^{\prime} \exp (i \Delta k \cdot z),
\end{aligned}
$$

where $E_{j}^{\prime}=n_{j} E_{j}$,

$\gamma_{i}$ is the absorption coefficient, $K$ is proportiona! to the nonlinear susceptibility, and $\Delta k=k_{3}-k_{2}-k_{1}$ is the phase mismatch. This set of equations is solved by integration over the thickness $z=L$ of the nonlinear medium.

The derivation and solution of these equations have been presented in detail in various articles and texts. ${ }^{7-16}$ Although such detailed discussions are beyond the scope of this article, we will draw upon those results to briefly discuss the factors that strongly impact the efficiency with which energy is transferred from the input waves to the generated wave at the sum frequency. As indicated by both analytical solution (assuming no absorption) $7,9,11$ and direct numerical integration of Eqs. (3)-(5), 10,12,17 the efficiency of sum frequency generation is governed primarily by two factors: (1) the phase mismatch between the input fundamental waves and the generated sum-frequency wave, and (2) the nonlinear drive, which is proportional to the product of the

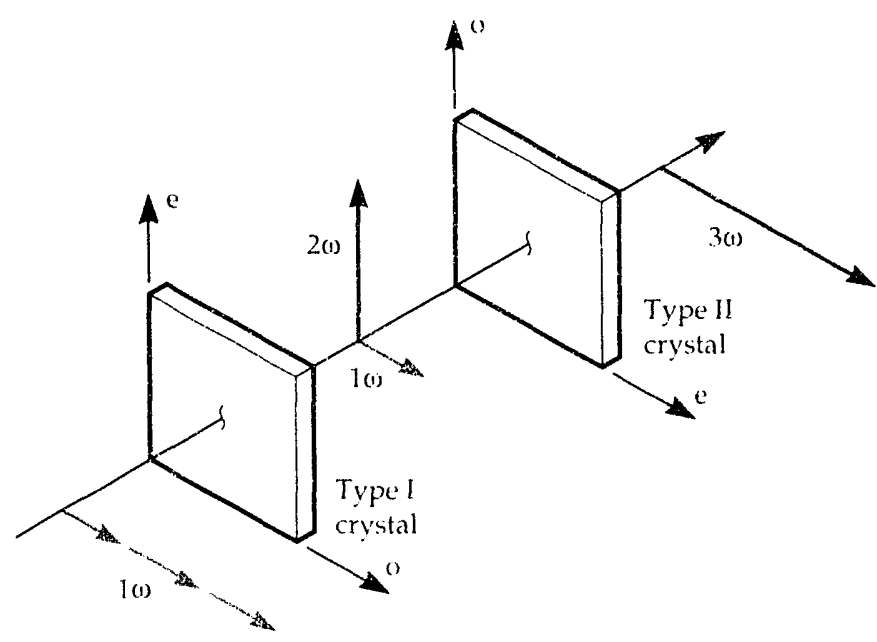

FIGURE 1. Schematic diagram of type 1/type II cascaded third harmonic generation. square of the nonlinear susceptibility, the square of the length of the nonlinear medium, and the sum of the intensities of the input waves. As the nonlinear drive increases, the conversion efficiency of the sum frequency generation process becomes increasingly sensitive to the balance between the input photon fluxes (mix ratio) and to phase mismatch. ${ }^{1(1-12,17}$

When perfect phase matching is achieved (i.e. $\Delta k=$ $0)$, the input and generated waves traverse the nonlinear medium with their phases locked together, allowing efficient power transfer from the input waves to the surn-frequency vave. When the phases of the three waves are not perfectly matched, power is cycled between the input and generated waves, resulting in a loss of conversion efficiency. This situation, termed back-conversion, is further exacerbated by unbalanced photon fluxes in the input waves. Phase matching is most commonly achieved by using the birefringence of optically anisotropic crystals to offset the phase mismatch caused by dispersion. ${ }^{18-20}$ Depending upon the refractive index dispersion of the crystal and the bircfringence available to offset it, two methods of phase matching are possible: type I phase matching, in which the two input waves have the same polarization, and type II phase matching, in which the two input waves are orthogonally polarized. Despite the fact that real laser beams, with phase, intensity, and polarization variations propagating through real crystals with defects, absorption, scattering, and inhomogeneities do not achieve these ideal conditions, efficient harmonic generation has nevertheless been accomplished. ${ }^{17,21,22}$

Efficient third harmonic generation is often realized by a sequential application of collinear sum-frequency mixing. A beam at the fundamental laser frequency is incident upon a nonlinear crystal in which second harmonic generation takes place via degenerate sum-frequency mixing. Two copropagating beams, one at the fundamental and the other at the second harmonic, emerge from the "doubling" crystal and are incident upon a second crystal in which the fundamental and the second harmonic waves interact with the nonlinear polarization of the crystal through the sum frequency mixing process to create a wave at the third harmonic. Several cascaded crystal schemes, classified by their phase matching methods, have been identified for implementing efficient third harmonic generation. ${ }^{10}$

Figure 1 shows a schematic diagram of type $I /$ type II third harmonic generation, which is the third harmonic generation scheme chosen for Beamlet and, consequently, the OSL experiments discussed below. The type I second harmonic generation crystal (the "doubling" crystal) converts a large fraction of the incident $1053 \mathrm{~nm}$ light to the second harmonic at $527 \mathrm{~nm}$. The second harmonic and the residual fundamental beams 
are then converted to $351 \mathrm{~nm}$ light by the type II mixing crystal (the "tripling" crystal). The efficiency with which the third harmonic is generated is very sensitive to the ratio of the intensities of the fundamental and second harmonic beams incident upon the tripler. This mix ratio is controlled by the length of the doubling crystal and the propagation direction of the fundamental wave relative to the phase matching direction inside the doubling crystal. For ideal plane waves incident upon a 10.5 - $\mathrm{mm}$ doubling crystal followed by a $9-\mathrm{mm}$ tripling crystal, the plane wave third harmonic conversion efficiency as a function of input intensity is shown by the plot in Fig. 2 . The crystal thicknesses and the angular detuning of the doubling crystal ( $250 \mu \mathrm{rad}$ from exact phase matching) are chosen to produce efficient third harmonic conversion for 1053-nm input intensities in the range of 2 to 6 $\mathrm{GW} / \mathrm{cm}^{2}$. Figure 3, a plot of harmonic conversion efficiency vs input fluence, shows the reduction in conversion efficiency that occurs for the temporal pulse shapes typical of the OSL laser (assuming that all other properties of the beam and crystals are ideal). Similarly, any departure from ideally perfect plane waves or harmonic generation crystals usually causes significant reductions in harmonic generation performance. The curves shown in Figs. 2 and 3 were calculated using a plane wave computer code used to model frequency conversion on Nova. ${ }^{17}$

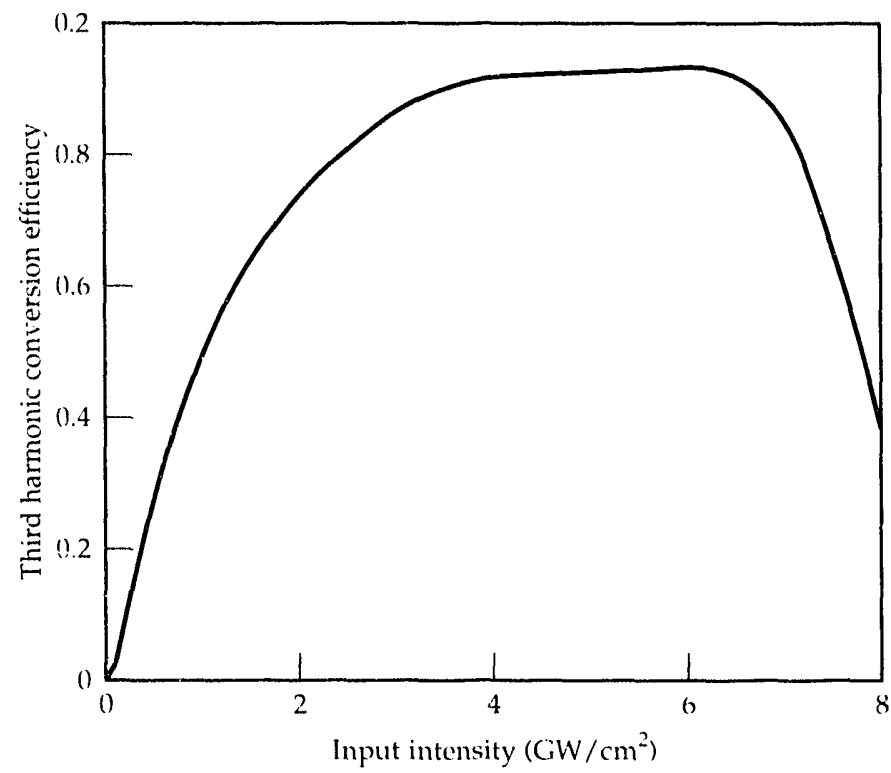

FICURE 2. Calculated third harmonic conversion efficiency as a function of the input intensity of a $1053-\mathrm{nm}$ ideal plane wave for a type $1 /$ type 11 phase matching scheme. The type 1 doubling crystal is 10.5 mm thick and is angularly detuned from exact phase matching by $250 \mu$ rad; the tripling crystal is $9 \mathrm{~mm}$ thick and is nearly perfectly phase matched.

\section{Harmonic Generation Experiments on OSL}

In order to study the performance of optical harmonic generation in KDP at fluences that previously would have damaged the conversion crystals, we have begun harmonic generation experiments on the OSL. The KDP crystals used in these experiments were $50 \mathrm{~mm}$ square by $8-11 \mathrm{~mm}$ thick. The input and output faces of the crystals are coated with a two layer antireflection (AR) coating: a thin layer of silicone is applied to protect the KDP surfaces, followed by a sol-gel AR coating. The sol-gel coating thickness on both faces of the doubling crystal and the input face of the tripling crystal is chosen to maximize transmission for light whose wavelength is $700 \mathrm{~nm}$. This provides a very good compromise for transmission both at $1053 \mathrm{~nm}$ and $527 \mathrm{~nm}$, and simplifies the coating process for the doubling crystal. The sol-gel coating thickness on the output face of the tripling crystal is optimized for maximum transmission of 351-nm light.

\section{Experimental Set Up}

The OSL generated the 1053-nm pulses that were used in this study of harmonic conversion. This laser consists of a single-mode Nd:YLF oscillator that emits

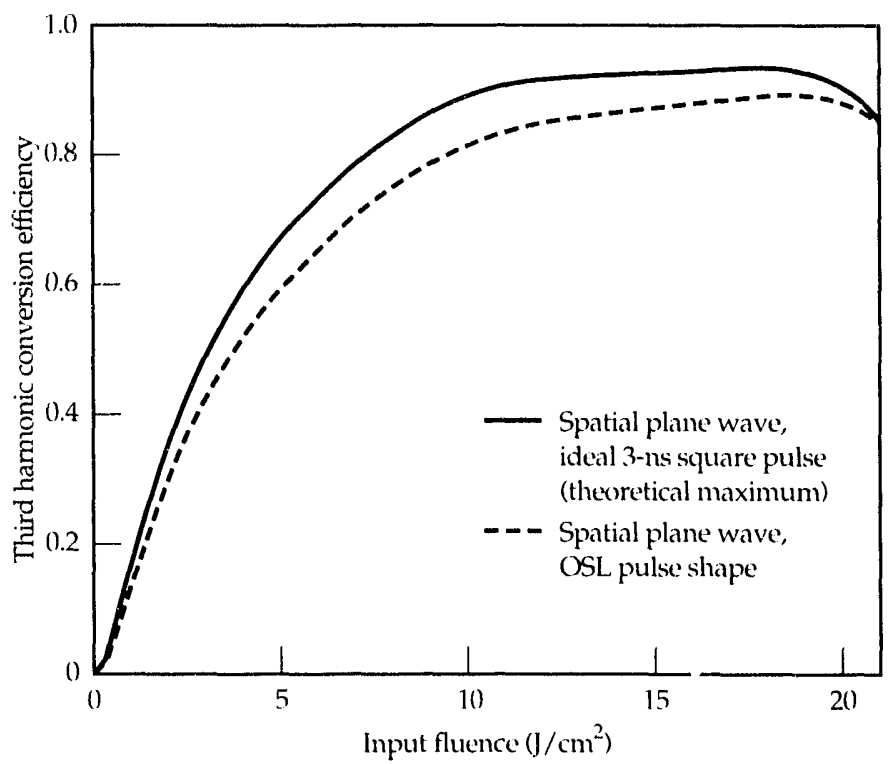

FIGURE 3. Calculated third harmonic conversion efficiency vs input fluence of $1053 \mathrm{~nm}$ laser pulse for a type l/type II phase matching scheme. The solid curve shows the performance of an ideally square 3-ns pulse, while the dashed curve indicates the harmonic generation performance with a temporal pulse shape typical of the OSL (as shown in Fig. 5). The crystal thicknesses and angular detunings are as given in Fig. 2. 
pulses 100 ns in duration, a two-stage Pockels cell shutter that allows creation of short pulses with almost rectangular waveform, and a chain of $\mathrm{Nd}$ :glass amplifiers with aperture to $10-\mathrm{cm}$ that allows the generation of pulses with energy to $100 \mathrm{~J}$. The beam is shaped spatially by using a serrated aperture to truncate the wings of the Gaussian beam emitted by the oscillator. In order to avoid excessive amplitude modulation that could damage amplifier chain components, an image of this aperture is relayed ${ }^{23}$ through the chain to the plane containing the KDP crystals. In the output plane of the laser, the beam is $80 \mathrm{~mm}$ in diameter. To generate the high fluences required for this experiment without damaging the laser, the beam diameter was downcollimated to $22 \mathrm{~mm}$ at the input to the experiment.

Figure 4 shows the arrangement of the conversion experiment. The main beam line contained the KDP crystals, two bare wedged silica splitters, a lens with focal length of $5 \mathrm{~m}$ and a CCD camera that was placed at the focus formed by the lens. The lens and camera were used immediately prior to a shot to reestablish beam pointing to within $10 \mu \mathrm{rad}$, but the camera was protected during shots by a beam block. A similar arrangement using the beams back-reflected from the crystals verified that the high-power beams followed the proper alignment path.

Five absorbing-glass calorimeters were used, and all of the optical components in the paths to these calorimeters were bare fused silica. Because beam polarizations are known for type I/type II tripling, the fraction of the energy arriving at each calorimeter could be accurately determined through Fresne? reflection and transmission calculations using measured values of the incidence and wedge angles for the silica components. Signals from the calorimeters were amplified by nanovolt meters, and recorded and processed by a Lab View program running on a desktop computer. All of the calorimeters were electrically calibrated to within less than $1 \%$. The calorimeters used to measure energy at $1053 \mathrm{~nm}$ and $527 \mathrm{~nm}$ were identical. The calorimeter used for 351-nm energy measurements, however, was unique (it was constructed with different absorbing glass). Excepting this one calorimeter, we could determine by permutation that the energy measurements of the other calorimeters and all the recording channels agreed to within $1 \%$. In order to make the harmonic generation measurements resemble more closely the plane wave model, apertures of $1.3-\mathrm{cm}$ diam were placed in the centers of the input and output diagnostic channels to strip away the spatially nonuniform intensities in the edges of the beam. Although the harmonic generation process took place in the crystals with the full spatial profile of the OSL beam, the calorimeters recorded only the energy transmitted by these apertures.

The shape of the input 1053-nm beam and the shapes at the output aperture of the three output beams were recorded by using silica lenses to image these beams onto CCD cameras. At low beam power, recordings of

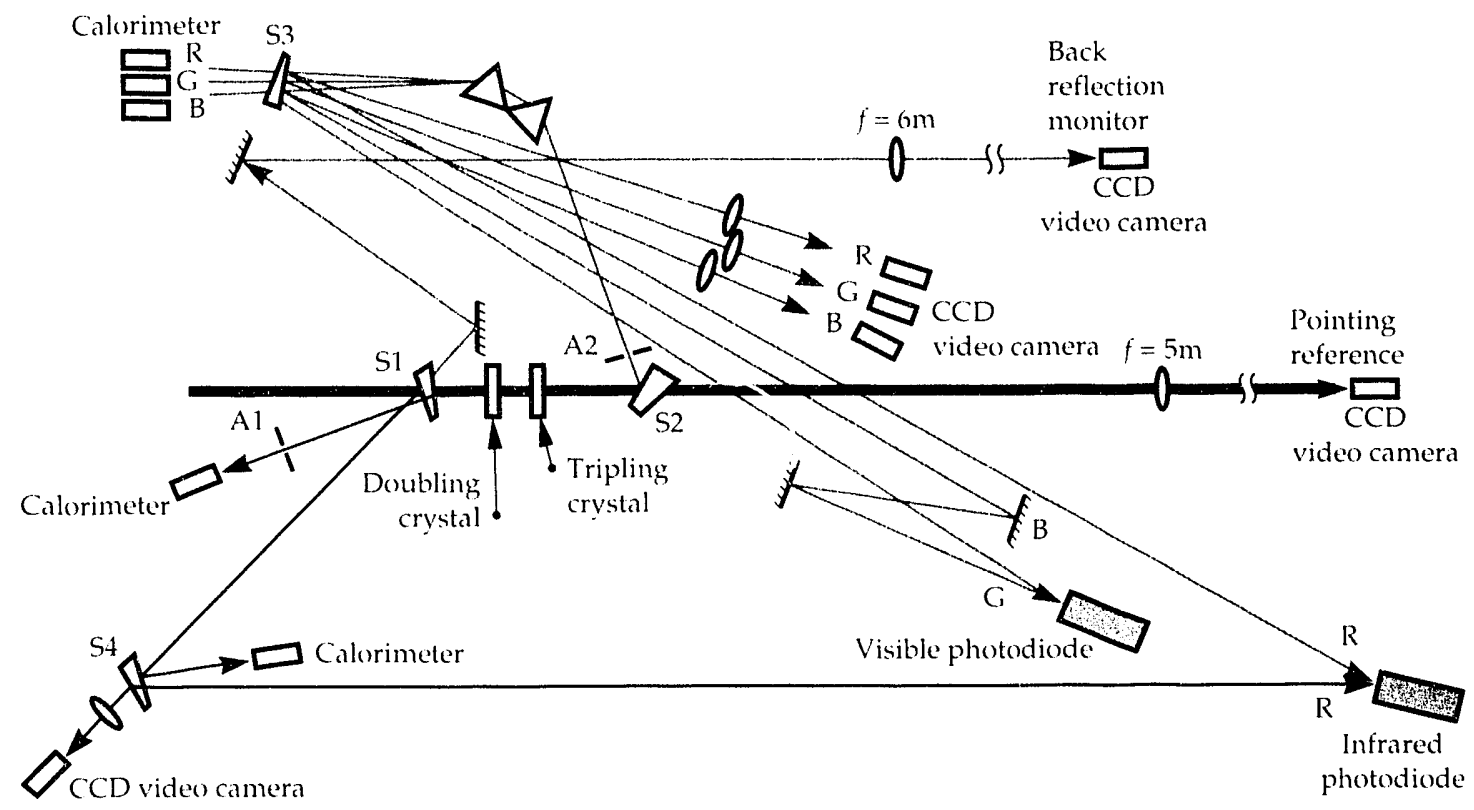

FIGURE 4. Experimental arrangement used at the output of the OSL to measure third harmonic conversion efficiency at high input fluences. The diagnostic beam paths have been somewhat simplified for diagram clarity. Symbols used: S1, S2, S3, S4-beam splitters; A1, A2-apertures; R-infrared beam (1053 nm); G-green beam (527 nm); B-ultraviolet beam $(351 \mathrm{~nm})$. The front and rear surface reflections of beam splitter $S 1$ feed the input infrared diagnostics. The front surface reflection from splitter S2 is dispersed by a prism pair, and each of the three wavelengths are directed to diagnostic instruments via transmission through splitter $\mathrm{S} 3$ and front and rear surface reflections from $\mathrm{S3}$. The small amount of infrared light reflected from the KDP crystals is used to monitor pointing of the beam to the crystals. 
resolution charts placed in object planes were used to certify the image quality. By time sequencing, a Hammamatsu diode and a Tektronix SCD5(1)(0) oscilloscope were used to record the waveforms for the input and residual 1053-12m pulses, and a similar arrangement was used to record the waveforms of the 527-nm and $351-\mathrm{nm}$ pulses.

The KDP doubler was installed first, preceded by a negative lens that was temporaril $y^{\prime}$ placed in the beam line. The diverging beam that emerges from the negative lens propagates energy over a range of angles. When this beam passes through the dubling crystal, the second harmonic conversion process take's place simultaneously over the range of angles contaired in the input beam. It forms a fringe pattern (Fig. 5) that is a single shot recording of the second harmonic generation angular tuming curve. The pattern was used to roughly align the phase matching direction of the crystal to the beam direction and to rotationally orient the crystal axes to the input polarization. The lens was removed, and a low-power angular tuning curve was produced to calibrate the crystal tuning mechanism and complete the adjustment of the sensitive tuning about the ordinary axis of the doubling crystal. The process was repeated to align the third harmonic crystal.

The doubling crystal was then rotated about its ordinary axis by $240-260 \mu \mathrm{rad}$, which is the angular detuning range predicted to produce high tripling efficiency with good dynamic range in input intensity.

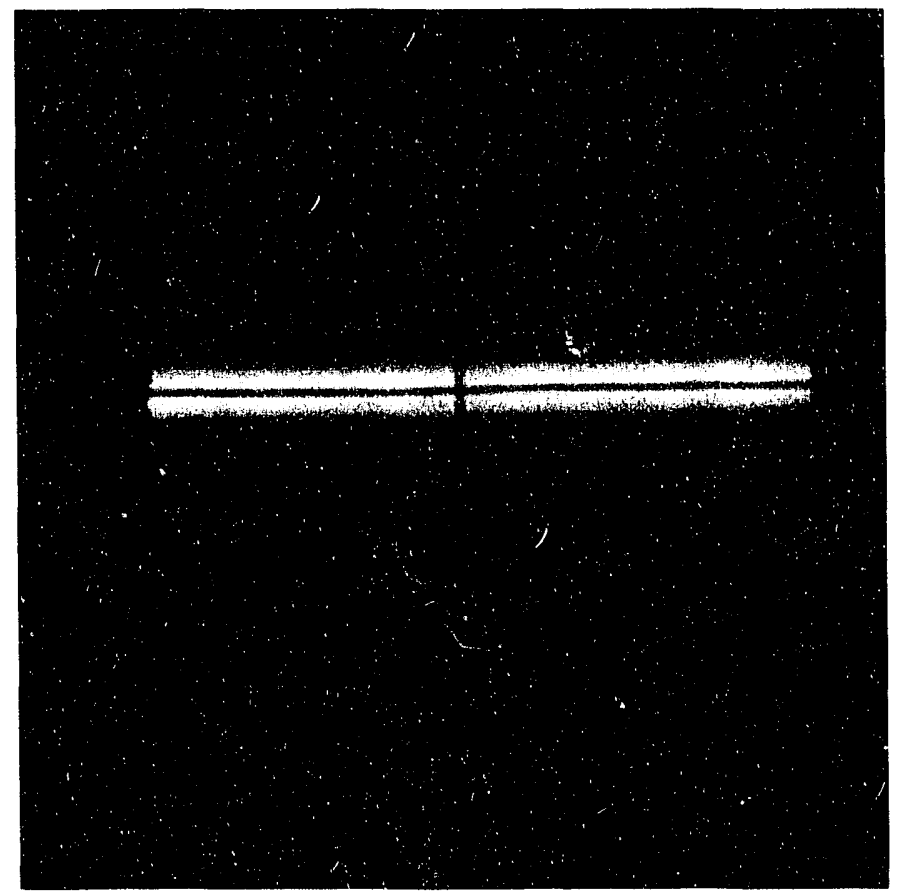

FICIURE 5. Photograph of the fringe pattern produced by second harmonic generation with a diverging input beam. This pattern is used with the crosshair visible in the photograph to coarsely align the doubling crystal.
The laser output at $1053 \mathrm{~mm}$ was then slowly increased from low to high fluences to condition the KDP crystais against optical damage. During and following the conditioning shots, the doubling and tripling crystal orientations were varied to establish experimentally the optimal crystal orientations and beam collimation conditions for third harmonic conversion. With these parameters optimized, the input fluence was increased until the experiment was terminated by laser-induced damage to the KDP tripling crystal and the fused silica beamsplitter that samples the output beam.

\section{Results}

The experiment described above had two different tripling crystals; a 9.12-mm-thick crystal for the first set of experiments and an 8.08-mm-thick crystal for the second set. The doubling crystal, whose thickness was $10.53 \mathrm{~mm}$, was the same for both experimerits. Aside from the thickness of the tripling crystal and some minor improvements in diagnostics, the primary difference between the experiments was the triggering method used for the two-stage Pockels cell shutter that shapes the laser pulses on their way to the amplifier chain. During the first experiment, the Pockels cell shutter was triggered from a laser-triggered spark gap. This arrangement produced the (normalized) optical pulse shape shown in Fig. 6. Before the second set of experiments began, the spark gap trigger for the Pockels cell shutter was replaced with an Auston-switch-based trigger that is initiated by pulses from a mode-locked oscillator synchronized to the single mode oscillator. This method of

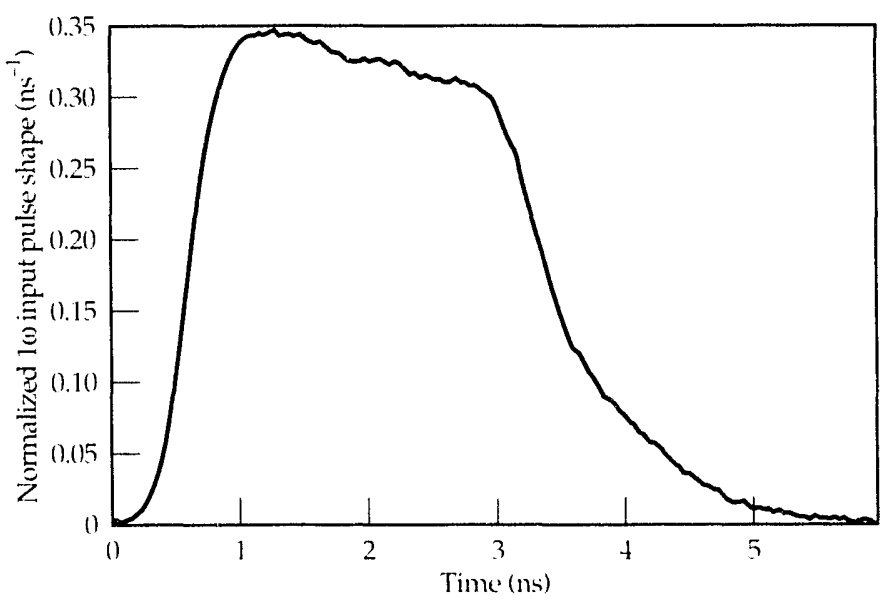

FICURE 6. Temporal shape of the $105.3-\mathrm{nm}$ laser pulse delivered to the KDP harmonic generation crystals during the first experiment. This pulse was selected as representative of the pulses produced by the Pockels cell shutter when it is triggered from a spark gap. The waveform shown is a pulse recorded by the oscilloscope and subsequently normalized to unit area. Multiplication of this waveform by fluence gives intensity as a function of time. 
triggering the Pockels cell provides pulses that are more square, i.e. that have sharper rising and falling edges and flatter tops; ultimately this method allows for more flexibility in pulse shaping. Figure 7 shows a normalized

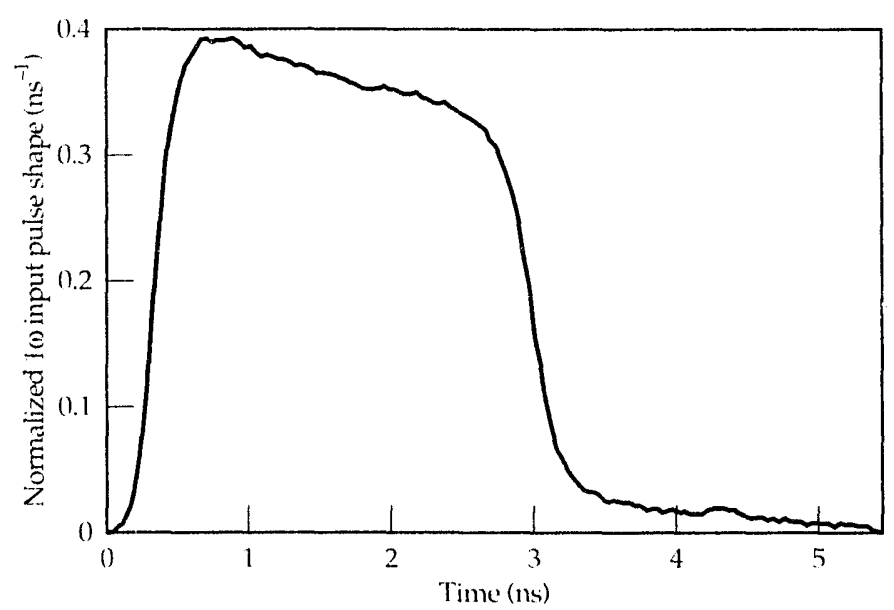

FIGURE 7. Temporal shape of the $1053-1 \mathrm{~mm}$ laser pulse delivered to the KDP harmonic generation crystals during the second experiment. This pulse is representative of the pulse's produced by the Pockels cell shutter when triggered by Auston switches. The waveform shown is a pulse recorded by the oscilloscope and subsequently normalized to unit area. Multiplication of this waveform by fluence gives intensity as a function of time. version of this pulse shape. Although both pulse shapes are approximately 3-ns square, the pulses produced from the Auston-switch trigger have steeper edgus than the pulses produced from the spark gap set up.

Information from all of the diagnostic instruments described above was recorded on every shot. The far field profiles were used to verify beam pointing directions in each crystal. The near field profiles and the waveform traces were examined for excessive spatial and temporal intensity modulation, so that meaningful average fluences could be used for calculating conversion efficiency. The incident and output fluences were calculated by dividing the measured energy that passed through the diagnostic apertures by the areas of those apertures. The energy balance was calculated by dividing the sum of the output fluences at all three wavelengths by the input fluence. This ratio is less than unity due to finite reflectivities of the crystal coatings and bulk absorption in the crystals. Harmonic generation conversion efficiency was calculated by dividing the output $351 \mathrm{~nm}$ fluence (or $527 \mathrm{~nm}$ fluence if only the doubling crystal was in place) by the input $1053 \mathrm{~nm}$ fluence.

Figures 8 and 9 give plots of several measured values of third harmonic generation efficiency vs input 1053-nm fluence. The data are grouped by internal
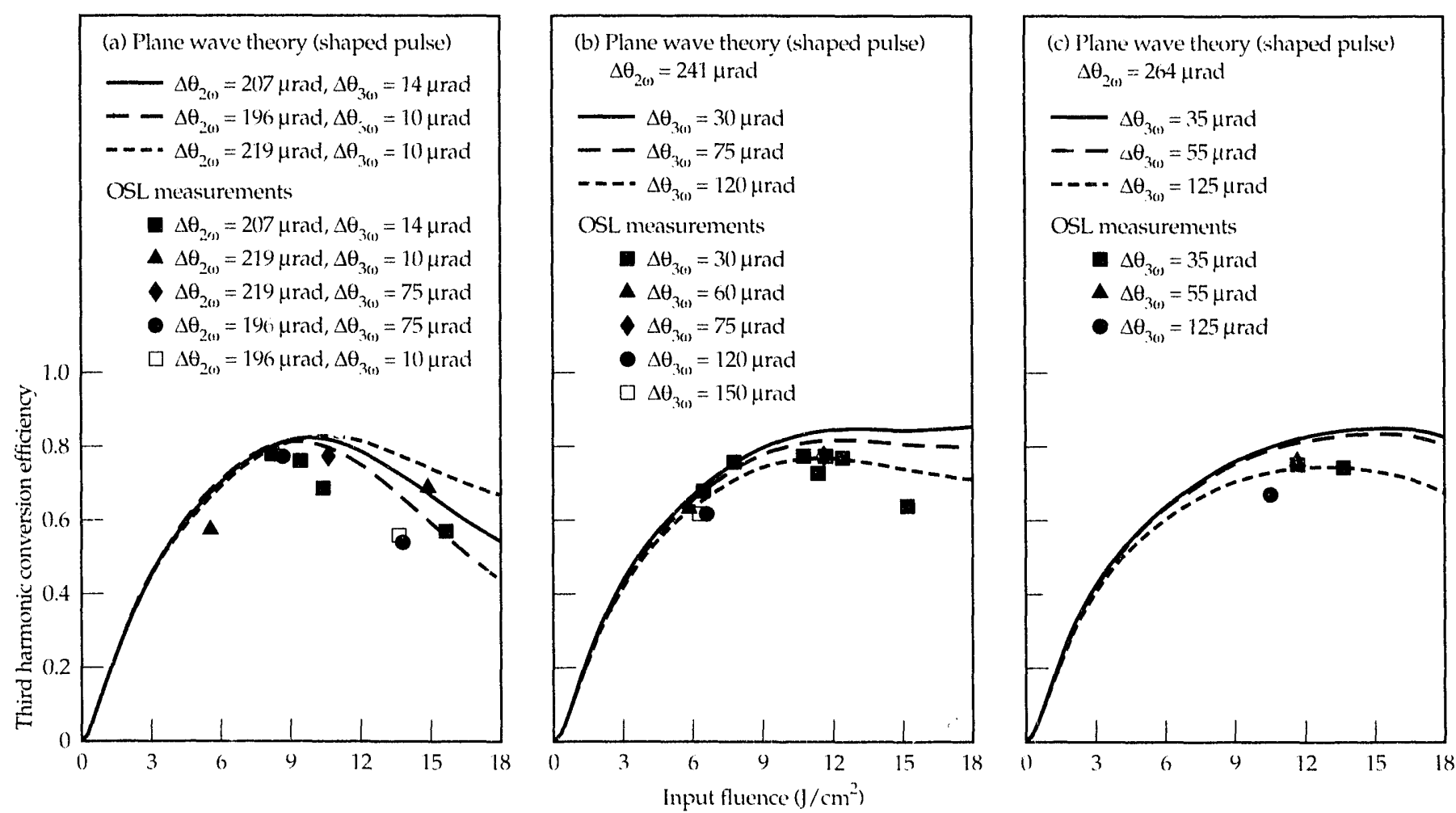

FIGURE 8. Calculated and measured third harmonic generation efficiency vs input fluence for type l/type Il phase matching. The doubling crystal was $10.53 \mathrm{~mm}$ thick and the tripling crystal was $9.12 \mathrm{~mm}$ thick. (a) Doubling crystal detunings of $196 \mu \mathrm{rad}, 207 \mu \mathrm{rad}$, and $219 \mu \mathrm{rad}$. (b) Doubling crystal detuning of $241 \mu$ rad with various tripling crystal detunings. (c) Doubling crystal detuning of $264 \mu$ rad with various tripling crystal detunings. For all three figures, the continuous curves give the plane wave conversion efficiency vs fluence for the input pulse shape shown in Fig. 5 , at the stated angular detunings. In the calculations, reflectivities of all four AR coated surfaces at all three wavelengths were assumed to be $0.5 \%$. 
propasation directions referenced from exact phase matching; $J \theta_{2(t)}$ is the propagation direction of the beam inside the doubling crystal and $.1 \theta_{3(1)}$ is the proposigation direction inside the tripling crystal. The continuous curves show'n in these plote are the third harmonic conversion efficiencies as a function of fluence that are predicted by the plane wave model for the pulse shapes shown in Figs. 6 and 7 , and the internal beam propagattion directions at which the data were recorded. These theoretical curves were obtained by (1) multiplying the nomalized input pulse shapes, as given in Figs o or 7 , by a fluence value to obtain input intensity as a function of time, (2) calculating the output pulse point-bypoint using the plane wave harmonic generation medel [Eys. (3)-(5)], (3) temporally integrating the output pulse to obtain an output fluence, and (4) dividing the output fluence by the input fluence to produce a conversion efficiency. Thus our model assumes a beam that is a plane wave in all respects except for a temporal pulse shape, which we have included by the procedure just described. This extension of the plane wave model is a good approximation for pulses whose spectral bandwidths are small enough to neglect the change in phase mismatch with respect to wavelength. As the plots indicate, there is reasonable agreement between the measured conversion efficiencies and the predictions of this simple extension to the plane wave model. At this point, we attribute most of the remaining discrepancy between the data and the model to characteristics of the beam and the crystals that we did not measure or include in the model: spatial variations in intensity; beam divergence due to finite beam size; phase front variations, which we nither measured nor modeled; nonlinear refractive inder effects in the orytals; and imperfect finishes and continges on the crystal surfaces. Preliminary measurements made with a recently activated interferometer indicate that the OSL beam used in the previous experiments had a $\lambda / 3$ bump in the phase front, which will impat the conversion efficiency. Nevertheless, we have demonstrated third harmonic comversion efficiencies of 70)-78", with input infrared fluences ranging from $6.5-13.5 \mathrm{~J} / \mathrm{cm}^{2}$.

\section{Summary}

Using 1053-nm-wavelength, approximately-3-nssquare pulses from the Optical Sciences Laser, we have produced third harmonic light in KDP' with efficiencies exceeding $70^{\circ} ;$ at input fluences approaching $14 \mathrm{~J} / \mathrm{cm}^{2}$. Extending the plane wave model to account for the nonideal temporal nature of the laser pulses gives reasonable agreement with the experimental results. By the end of each of the experiments whose results have been presented here, the fused silica output beamsplitter had accumulated massive optical damage. The KDP tripling crystals, however, displayed much less damage, and the doubling crystal, which was used in both experiments, showed only slight damage.

We are now directing our efforts toward extending our experimental and modeling capabilities. An interferometer is being added to the input diagnostic package, although initially its output will not be recorded on every shot. A multipass phase modulator has been added between the oscillator and amplifier chain to
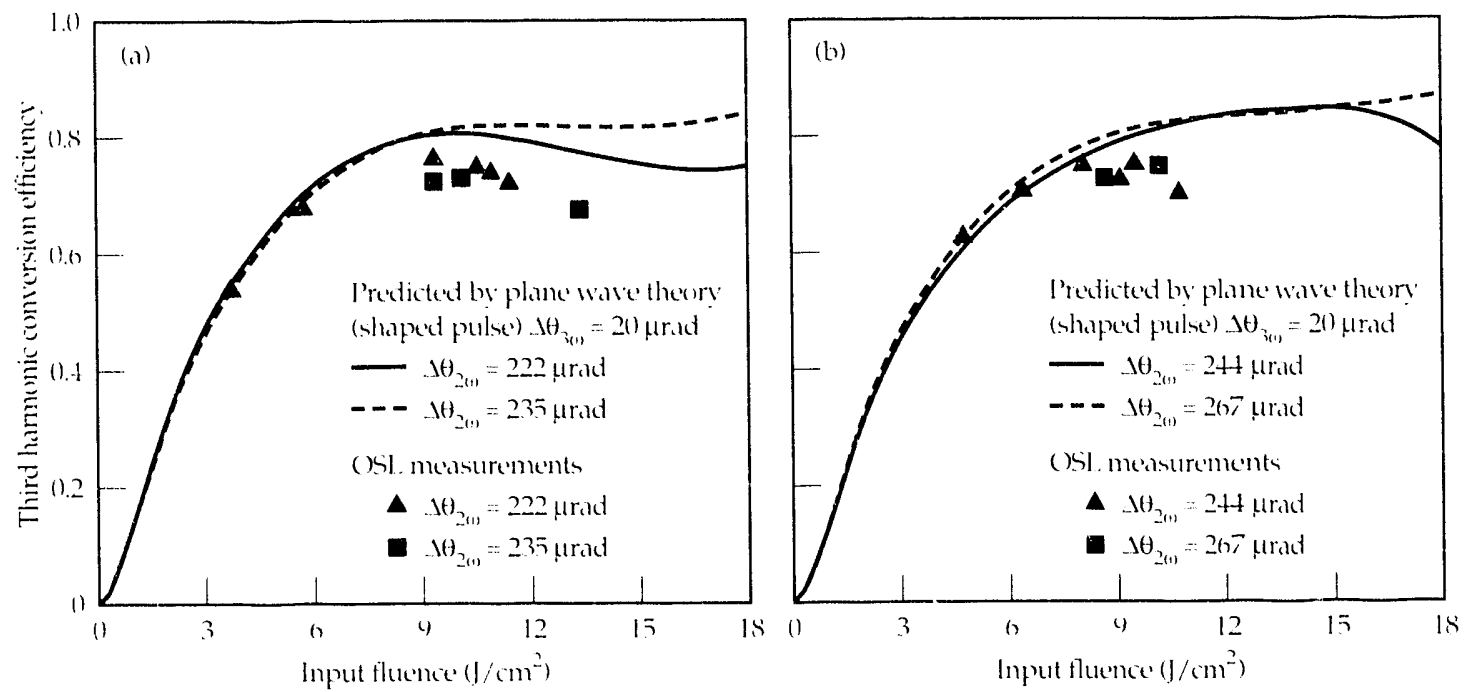

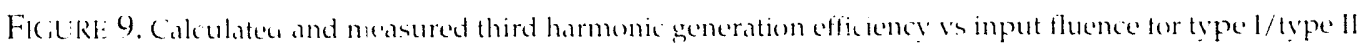
phose motehing. The doubling orystal was $10.53 \mathrm{~mm}$ thich and the iripling crystal was $8.18 \mathrm{~mm}$ thich. (a) Doubling cristal detumings of $222 \mu \mathrm{rad}$ and $235 \mu \mathrm{rad}$. (b) Doubling crystal detuming of $24+4$ rad and $26 \mathrm{arad}$. For both fig. ures, the continumus curves give the plane wase conversion efficiency a fluence for the inpul pulse shape shown in Fig. h, at the stated angular dettinings. In the calculations, reflectivitien of all four AR conted surfaces at all three wavelengtho were astumed to be $11.5 "$. 
provide spectral bandwidth to the i)53-1um beam. Diagnostics to support this band width capability are also being added. Early streak camera results indicate that the laser pulse shape has sharper leading and trailing edges tinan Fig. 7 indicates. New frequency conversion codes, $\therefore$ ich are near completion, will allow us to model the effects of imperfect phase fronts and the addition of spectral bandwidth.

\section{Acknowledgments}

We would like to thank D. Eimerl, M. Henesian, and J. R. Murray for helpful discus sions, B. Olejniczak and M. Webb for their help in performing these experiments, and $K$. Stanion for coating the KDP crystals.

\section{Notes and References}

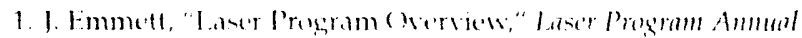

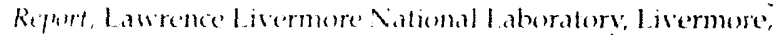

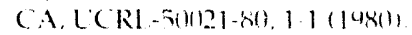

2. M. A. Summers, L. (i Seppola and I. 1) Williams, "Now

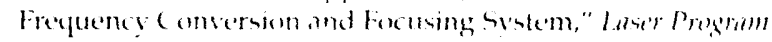
Ammal Repent. lawrence livemore National l aboratory"

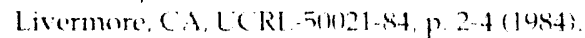

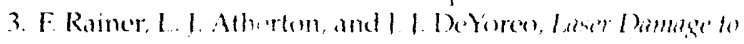

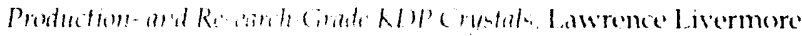

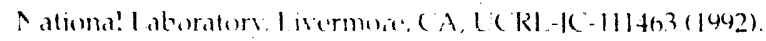

4. M. R. Koylowhi. I M. T'.umas I. II Compledl, and F. Rainer.

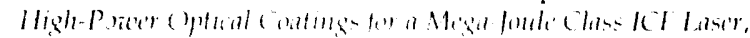
Lawrence Livermore National l aborators, Livermore, CA,

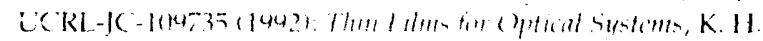

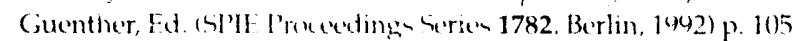

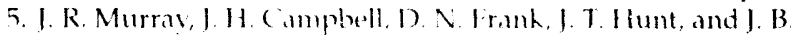
Trenholme. "The Noxial pegrate Btamlet Demonstration

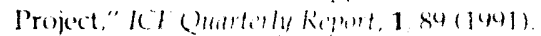

6. D. Eimerl, R. Bowd, and I) Milam, "The Optical Soiences

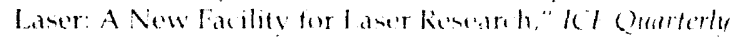

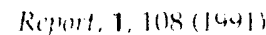

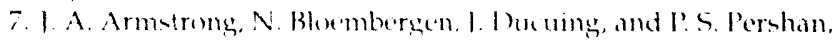
"Interations between light Waven m a Nomlinear Dielectric," Plus Row, 127, 1918, 1902).
8. P. N. Butcher and D. Cotter, The Elements of Nomlinear Optics (Cambridge University Press, Cambridge, 1990).

9. D. Eimerl, "Electro-Optic, Linear, and Nonlinear Optical Properties of KDP and Its Isomorphs," Ferroclectrics, 72, 95 (1987).

10. R. S. Craxton, "High Efficiency Frequency Tripling Schemes for High-Power Nd:Glass Lasers," IEEE I. Qututum Elo'tron., QE17, 1771 (1981).

11. D. Eimerl, "High Average Power Harmonic Generation," IEEE I. Qututum Electrum., QE-23, 575 (1987).

12. R. C. Fckardt and J. Reintjes, "Phase Matching Limitations of High Efficiency Second Harmonic Generation," IEEE I. Qumutum Eloctron., QE-20, 1178 (1984)

13. F. Zernike and J. E. Midwinter, Applied Noulinear Optics (John Wiley \& Sons, Inc., New York, 1973).

14. A. Yariv, Qunntum Electronics, 2 in Edition (john Wiley \& Sons, Inc., New York, 1975).

15. Y. R. Shen, The Principle's of Nonlinear Optics (fohn Wiley and Sons, Inc., New York, 1984).

16. R. W. Boyd, Nomlime?r Optics (Academic Press, San Diego, 1992).

17. M. A. Jenesian, I'. J. Wegner, D. R. Speck, C. Bibeau, R. B. Ehrlich, C. W. Laumann, J. K. Law'son, and T. L. Weiland, "Modeling of Large Aperture Third Harmomic Frequency Conversion of High lower Nd:Cihss 1 aser Systems," Motding and Simulation of Lasor Systimts II, Lawrence Livermore National Laboratory, Livermore, CA, UCRL-IC-104434 (1991); SPIL: Pro: 1415 (1991).

18. J. A. Ciordmaine, "Mixing of Light Beams in Crystals," Phys. R'í. Liff., 8, 19 (1962).

14. P. D. Maker, R. W. Terhune, M. Nisenoff, and C. M. Savage, "Effects of Dispersion and Focusing on the Production of Optical Harmonics," Phys. Ret. Left., 8, 21 (1962).

211. J. E. Midwinter and J. Warner, "The effects of phase matching method and of uniaxial crystal symmetry on the rolar distribution of second-order non-linear optical polarization," Brit. I. Apll. Phy. 16, $1135(1965)$.

21. I?. J. Wegner, M. A. Henesian, D. R. Speck, C. Bibeau, R. B. Ehrlich, C. W. Lammann, J. K. Lawsom, and T. L. Weiland, "Harmonic Comversion of Large Aperture 1.(5- $\mu$ m Laser Beams for Inertial Confinement Fusion Research," Appl. ( )pt., 31, 6414 (1992).

22. W. Seka. 6. D. Jacobs, J. E. Rizzo, iR. Boni, and R. S. Craxton, "Demonstration of High Efficiency Third Harmonic Conversion of High Power Nd-Cilass Laser Radiation," ()pt.Commmun., 34, $469(198())$

23. I. T. Ifunt, J. A. Cilaze, W. W. Simmons, and P. A. Renard, "Suppression of self-focusing through low-pass spatial filtering and relay imaging," Appl. ()pt., 17, 2(15.3 (1978). 


\section{Ultraviolet InduCED Transifnt Absorption IN KDP AND ITS INFLUENCE ON FOURTH HARMONIC FREQUENCY CONVERSION}

C. D. Marshall

H. W. H. Lee*

J. E. Davis ${ }^{\dagger}$

S. A. Payne

J. A. Speth

R. S. Hughes, Jr.*

\section{Introduction}

Over the past decade, there has been considerable investication of frequency conversion into the ultraviolet for fusion laser systems. Either deuterated or undeuterated potassium dihydrogen phosphate crystals (KD*P and KDP) have been the materials of choice, since they can be grown to the requisite large apertures with reasonably high laser-damage thresholds. A number of experimental frequency conversion studies from the second harmonic $(2 \omega)$ of $\mathrm{Nd}^{3+}: \mathrm{YLF}$ or $\mathrm{Nd}^{3+}:$ YAG at $0.53 \mu \mathrm{m}$ to the fourth harmonic $(4(1))$ at $0.26 \mu \mathrm{m}$ have been performed using KDP with varying results. ${ }^{1-7}$ In the past, there has been some concern regarding the mechanisms responsible for the low conversion efficiency to the fourth harmonic at high $\left(I_{2(1)}>2 \mathrm{GW} / \mathrm{cm}^{2}\right)$ drive intensities and the temporal distortions in the $4 \omega$ beam. Most reported descriptions of these mechanisms have relied upon either unusually large 40 two-photon absorption constants of $>0.4 \mathrm{~cm} / \mathrm{GW}^{1,2,5,6}$ or large crystal angle detuning of $>100 \mu \mathrm{rad}^{4}$ to explain the conversion efficiency curves. These explanations, however, have not yielded a consistent picture of the experimentally observed conversion efficiency and pulse shaping effects.

This paper quantitatively addresses frequency conversion to $4(1)$ by including the effect of a recently observed two-photon induced transient broadband $(250-700 \mathrm{~nm})$ absorption intrinsic to KDP and $K D^{*} P^{X-10}$ Under intense ultraviolet (UV) irradiation from 4 to $h$ eV, KDP undergoes significant two-photon absorption producing electrons in the conduction band. Some of the energetic electrons released from this process go on to yield defect states in the material. The defects create a transient gray track in the sample wherever the UV

\footnotetext{
* Photenica (iroup, Nuclear Tent Experimental sience Division t Department of Applied science, Univernity of California at Davis
}

beam has passed through the crystal (Fig. 1). A probe laser beam subsequently experiences up to $50 \%$ absorption for a 1-cm-thick crystal, depending on the specific wavelength, intensity, and temporal pulse shapes of the (a)

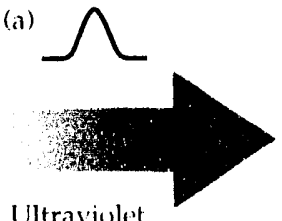

Ultraviolet excitation pulse

(b)

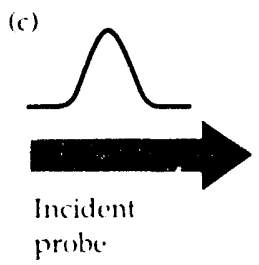

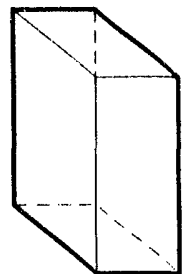
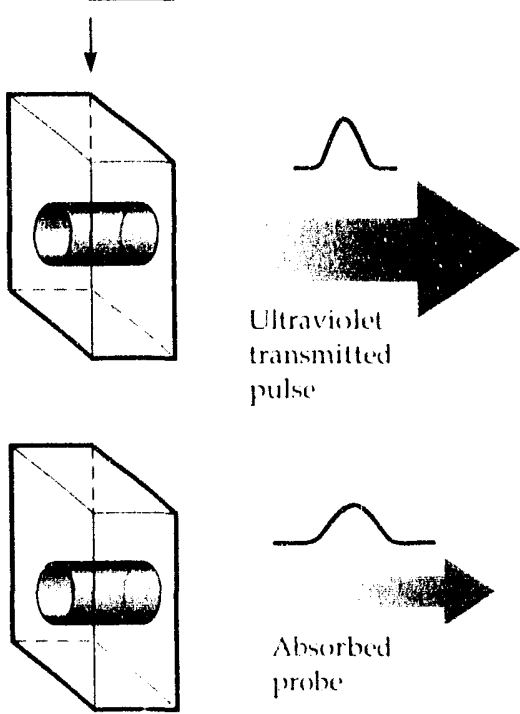

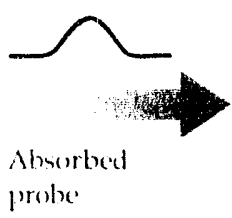

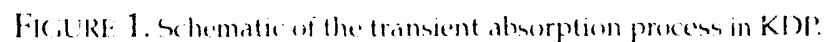
(a) and (b) show how an intense Le berm an produce long lived

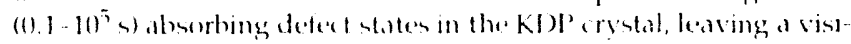

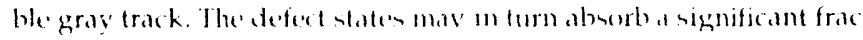
tion $(-50 \%)$ of the probe light pulse a shown in (c). 
pump and probe beams. In general, the optical density builds up during the laser pulse and is directly related to the time-integrated UV intensity that has irradiated a given point in the crystal. This gray track or transient solarization relaxes in $10^{-1}$ to $10^{5}$ seconds, leaving the sample optically transparent again.

We discuss the formation and decay kinetics, as well as a plausible physical model that describes the transient absorption phenomena. The results of this analysis have been incorporated into an existing frequency conversion code. 11 For a modest $1 \mathrm{GW} / \mathrm{cm}^{2} 2(1)$ drive intensity and a $1 \mathrm{~cm}$ crystal thickness, the maximum conversion efficiency is reduced only $\sim 2 \%$ by the inclusion of transient absorption. However, when the $2(1)$ drive intensity is raised above $3 \mathrm{GW} / \mathrm{cm}^{2}$, the transient absorption has a significant impact and the $4(1)$ conversion efficiency can be reduced by more than $50 \%$ as experimentally observed. ${ }^{5,6}$

\section{Transient Defect Absorption Experiments}

Three excitation and three probe sources were used in a variety of combinations. The first excitation laser, consisting of an amplified Q-switched Nd:YAG unstable resonator laser and frequency conversion crystal array, produced $>20 \mathrm{~mJ}$ of 40 and $3($ a energy at $10 \mathrm{H} /$ in a highly structured temporal pulse shape which had a $1 / \mathrm{e}$ full-width of $10 \mathrm{~ns}$ when fit to a Gaussian curve. The second excitation source, a mode-locked Nd:YAG laser, produced 100 ps pulses at $76 \mathrm{MHz}$. The pulses were amplified in a 10-Hz Nd:YAC, regenerative amplifier system and frequency converted to produce $\sim 15 \mathrm{~m}$ ] at $4(1)$. A third pump source, a hybrid mode-locked

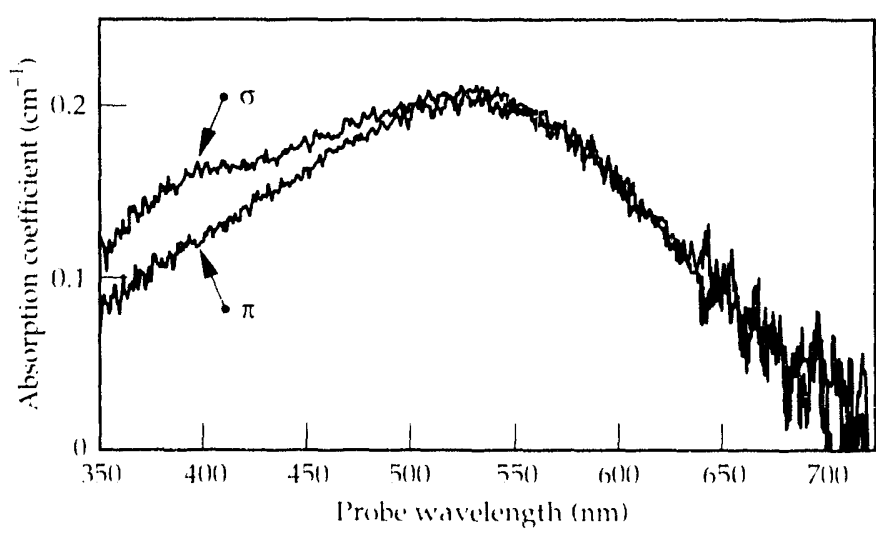

FIC iLRI: 2. Time-resolved polarized absorption spectrum of LV induced trameient defects in 1.5-cm-thick KDl'orseal haken-1 ms after excitation at $260 \mathrm{~mm}$ with $\left.-51 / \mathrm{cm}^{2},-11\right)$ m pulses. The pump pulse was polariad perpendicular to the optionl axis of the crental and the spectrum was measured with a Xe are lamp polarised either

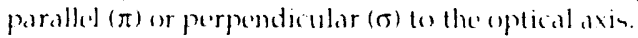

sub-picosecond dye laser system that was pumped and amplified by the $2(1)$ output of the 100 -ps system, was frequency converted to produce $\sim 25()$-fs pulses at $308 \mathrm{~nm}$ and $10 \mathrm{~Hz}$. To generate transient absorption in the crystal, the pump sources were focused to $\sim 250 \mu \mathrm{m}$ $1 / \mathrm{e}^{2}$ diam Gaussian spot sizes in the test sample and attenuated to $0.01-1 \mathrm{~mJ}$ (to remain below the catastrophic laser damage threshold). The first probe source, a continuous wave (CW) Xe arc lamp whose spectral absorption was monitored with a $0.32 \mathrm{~m}$ monochrometer and optical multichannel analyzer system gated $\sim 1 \mathrm{~ms}$ after the pump beam reached the test sample, provided a spectrum of the induced transient absorption. The second probe source, a 25()$-\mathrm{fs}, 308 \mathrm{-nm}$ beam that was temporally delayed relative to the pump beam, nonitored the formation time of the transient absorption. The third probe source, a green CW HeNe laser operating at $543.5 \mathrm{~nm}$, monitored the decay dynamics of the absorption. All three probe beam sources were focused to a $10(0)-\mu \mathrm{m} 1 / \mathrm{e}^{2}$ diam spot size and overlapped with the pump beams in the sample. The beam profiles were monitored and recorded using a video camera system. The transient absorption was monitored using three photodetectors connected to gated integration electronics, a digitizing oscilloscope, and a computer.

\section{Transient Defect Absorption Spectrum}

To measure its transient absorption spectrum, we excited the KDP with $\sim 5 \mathrm{~J} / \mathrm{cm}^{2}$ of 40 light produced by the Q-switched Nd:YAC laser system (or first excitation laser) and obtained measurements with the spectrometer system described above. Figure 2 shows the polarized spectra, both parallel and perpendicular to the axis of the crystal. The absorption extends from the UV to the near infrared with a broad $(\sim 250) \mathrm{nm}$ wide) roughly Gaussian peak centered around 5()()-550 $\mathrm{nm}$, the exact position of which depends upon the particular sample under study. A weaker second peak $(\sim 10) \mathrm{nm}$ wide) is centered at $390-410 \mathrm{~nm}$.

This spectrum is very similar to that observed in KDI' after bombardment with electrons or $x$-ray and $\gamma$-ray radiation. ${ }^{12-18}$ Under those conditions, crystalline defect sites, identified as $\mathrm{HPO}_{4}$ - radicals via electron spin resonance spectroscopy, were formed and broad optical absorption was simultaneously observed. Even though the electron energies produced by 40 two-photon absorption are significantly lower, there is sufficient energy to bridge the optically transparent band gap of KDI' and liberate an excited electron. The similarity of the UV and ionizing-radiation-induced absorption spectra suggest that both absorptions arise from the same general classes of defect states. 


\section{Defect Formation and Relaxation Dynamics}

To measure the formation time of the transient defect absorption effect in KDP, we devised a pumpprobe experiment, where the transient defect absorption was generated and probed with the 250-fs, 308- $17 m$ amplified dye laser system described above. Figure 3 shows the results. Tle defect formation dynamics were modeled by assuming that the transient optical density followed the form $\left[1-\exp \left(-\right.\right.$ time $\left.\left./ \tau_{f}\right)\right]$, where the formation time $\tau_{f}$ was determined to be $1.6 \pm 0.4 \mathrm{ps}$. ${ }^{10}$ This formation time can be considered instantaneous relative to the 10()-ps to 10-ns excitation pulse lengths used throughout the experiments described in the remainder of this paper and the long ( $>1$ s) absorption relaxation times observed.

We measured the temporal decay rate of the induced absorption by monitoring the change in transmission of the 54.3.5-nm HeNe probe laser after excitation with $\sim 6$ $\mathrm{J} / \mathrm{cm}^{2}$ of $4(1)$ light from the Q-switched Nd:YAG, laser system. Data were obtained on seven different KDI' crystals $(a-g)$, five different KD*P crystals $\left(a^{*}-e^{*}\right)$, one rubidium dihydrogen phosphate crystal (RDI'), and one ammonium dihydrogen phosphate crystal (ADP). The decay times of the transient absorption varied by six orders of magnitude from 0.2 to $4 \times 10^{5} \mathrm{~s}$ for different crystals, although the peak absorption strength at zero time delay was indistinguishable for the same excitation conditions. The decay times were completely uncorrelated with either deuteration level or cation composition. Figure 4 shows representative plots of the absorption change at $543.5 \mathrm{~nm}$ vs time after $4(1)$ pump excitation for samples $a^{*}, b$, and $c^{*}$ corresponding to 55,6 , and $0.3 \mathrm{~s}$ $1 /$ e decays respectively. Since there was no correlation of the decay rates with the deuteration level of the crystals, one might hypothesize that impurities were responsible for the widely varying decay rates.

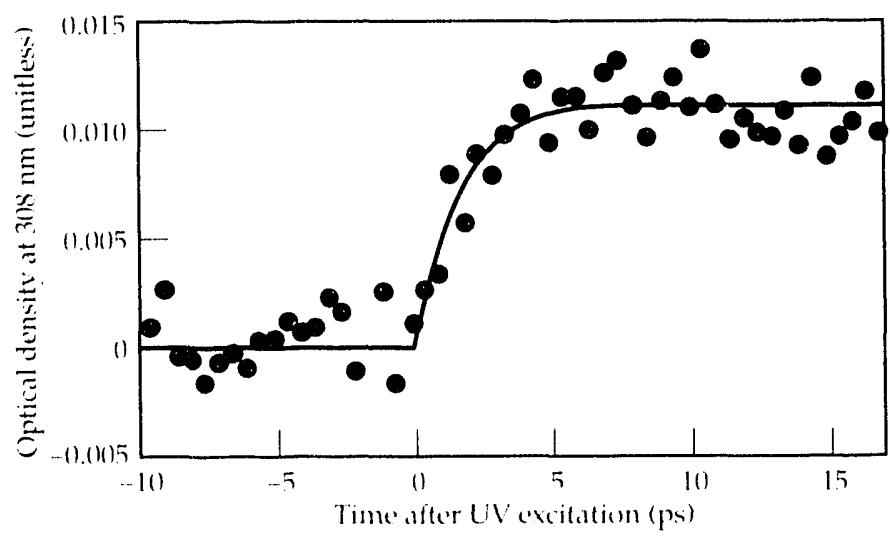

FIC:LER 3. Time resolved pump-probe experiment performed an a 1.7-cm-thick KI)]' (rystal with 308-nm, 25(1)-1s pulses for monitor the formation of the aturorbing defects. The solid line through the data is at tit to determine the tramsient absorption formation time of lon ps.
To investigate the relationship, if any, oetween the relaxation time of the transient absorption and known sample impurities, mass spectral analysis on aqueous solutions prepared from the 14 different crystal samples was performed on 20 elements of interest to the KDI' crystal growth industry. ${ }^{19}$ The decay times were uncorrelated with $\mathrm{As}, \mathrm{Pb}, \mathrm{Ba}, \mathrm{Sr}, \mathrm{Rb}, \mathrm{Cu}, \mathrm{Cr}, \mathrm{V}, \mathrm{Al}, \mathrm{Mg}$, $\mathrm{Na}, \mathrm{B}$, and Li impurities. The elements Fe, Cl, S, Ni, Ti, $\mathrm{Ca}$, and $\mathrm{Si}$ were below the experimental detection limit; therefore, the relation between decay time and sample contamination could not be determined for these elements. Extrinsic impurity absorptions from a variety of elemental impurities have been observed in the literature in irradiated KDP and its isomorphs but these impurities predominantly led to absorptions in the UV portion of the spectrum. 21-27 Thus it appears that the transient defect absorptions observed in the visible portion of the spectrum are umrelated to the above impurities. As discussed below, the variation of the decay rates is determined by the concentration of intrinsic imperfections, or traps, in the crystal. We have not found these imperfections to be related to the impurities noted above.

Figure 5 shows a temperature-dependent plot of the transient absorption decay rate. A decay time $\tau_{d}$ or inverse decay rate was evaluated at each temperature Thy integrating each absorption decay, similar to those shown in Fig. 4, over time and dividing by the peak optical density. This measure of the decay time is independent of any physical model used to describe the thermally assisted decay kinetics. A linear least syuares

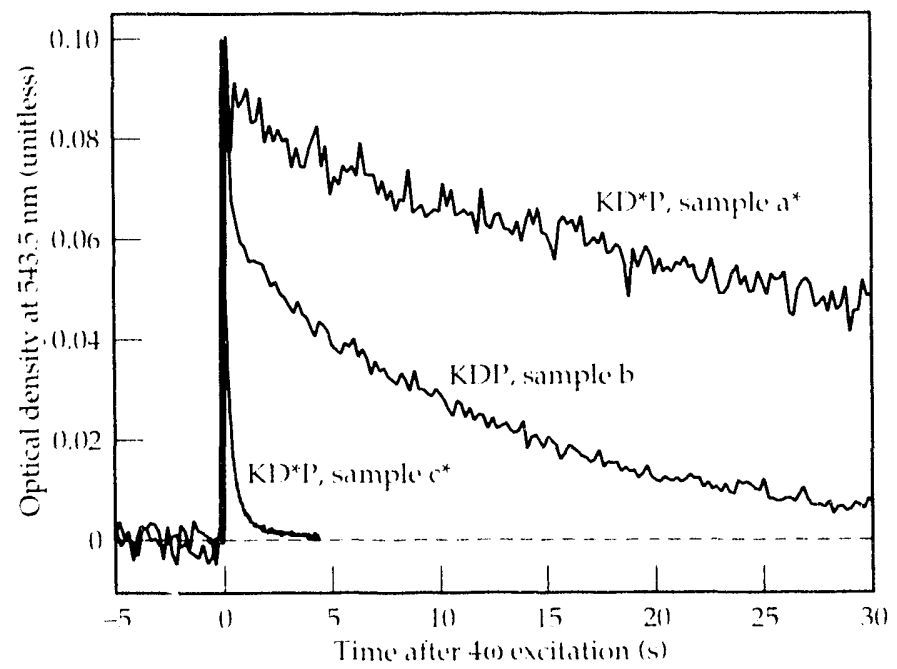

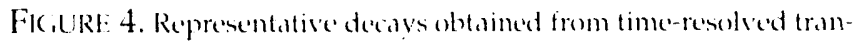
sient abserption experiments performed on one KD)l (b) and two KI $)^{*} P^{\prime}\left(a^{*}, c^{*}\right)$ samples simm thich. Absorption decal's were measured

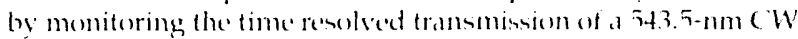
I HeNe laser following pulsed LIV ancitation. 
was fit to the result to determine the slope gives an Arrhenius activation energy $E_{\mathrm{a}}$ defined by

$\tau^{-1}=\tau_{\mathrm{d}}^{-1} \exp \left(-E_{\mathrm{a}} / \mathrm{k} T\right)$

We found that $E_{\mathrm{a}}=0.51 \pm 0.04 \mathrm{eV}$ for all ADP, KDP, and $K D * P$ crystals, and $E_{\mathrm{a}}=0.85 \pm 0.04 \mathrm{eV}$ for the RDP crystal. Since the absolute decay times varied by several orders of magnitude at a given temperature for individual samples but the activation energies remained constant for samples with the same chemical content, this indicates that a thermally assisted mechanism controls the absorption decay kinetics in all the materials studied. The comparable activation energies also indicate that the physical mechanism limiting the decay rates, discussed below, is identical for all the KDP and KD*P samples, and is similar to that in the RDP and ADP crystals.

\section{Intensity Dependence \\ of Transient Absorption}

We measured the strength of the induced absorption $\sim 1 \mathrm{~ms}$ after initial excitation as a function of pump fluence for both 40 and 30 excitation with 100 -ps and 10ns pulse lengths. Four different KDP crystals (a, d, f, and $g)$, and a single $K D^{*} P$ crystal $\left(d^{*}\right)$ were studied. The transient absorption strength was monitored by measuring the transmission of the green $(543.5 \mathrm{~nm})$ HeNe laser, polarized both parallel and perpendicular to the optic axis, with a digitizing oscilloscope. Figures $6(a)$ and (b) show data taken with 10-ns and 10()-ps laser pulses,

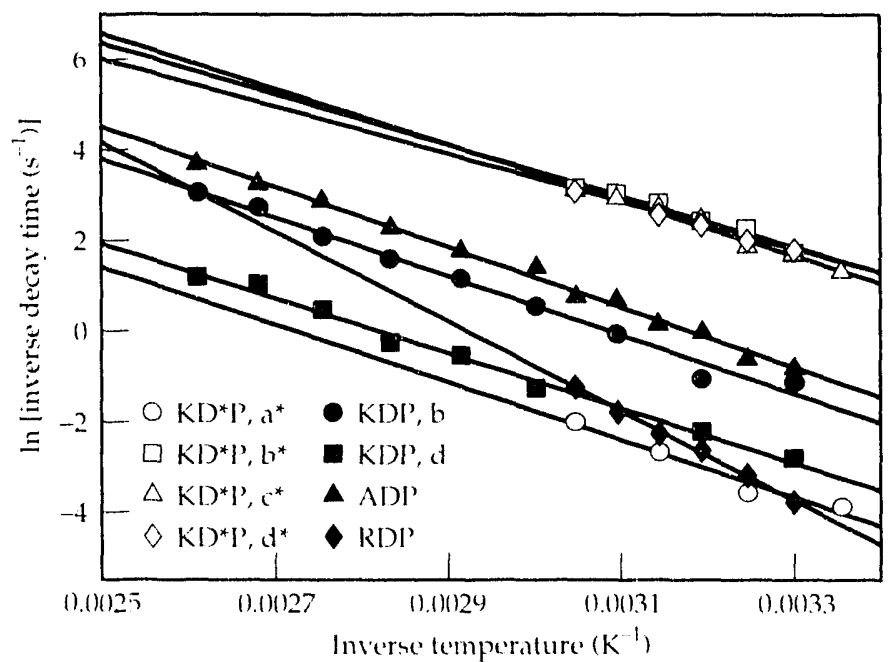

FICiURE 5. Temperature dependence of absorption decay rate for se'eral KD*L', KDI, ADI', and RDI' namples 8 mm thick. The linear natue of the temperature dependencies indicales that a thermally activated process is responsible for the abourption decay mechanism. The slopes are proportional to the Arrhenius activation energies determined by Eq. (1). respectively. The solid lines are numerical fits to the data discussed below. The data clearly exhibit a quadratic region at low intensity, indicating that Iwo-photon absorption is initially responsible for creating the defects. The linear dependence of the absorption at higher intensities indicates that saturation or bleaching of the defect states occurs, as quantitatively modeled below. This bleaching is not surprising since absorbing defects, generated with ionizing radiation, are known to bleach under UV irradiation from a 50(1)-W Hg arc lamp. ${ }^{14}$ Under the relatively intense irradiation of $1-20 \mathrm{GW} / \mathrm{cm}^{2}$ used in our experiments, we could expect defect formation via two-photon absorption to dominate until a sufficient fluence had irradiated the sample during the laser pulse. At the very highest fluences with 100 -ps pulses in Fig. 6(b), two-photon absorption should deplete the 40 drive pulse, leading to a large rollover in the induced transient absorption. Note that the $3(1$-induced absorption in Fig. 6(a) is substantially weaker than that obtained
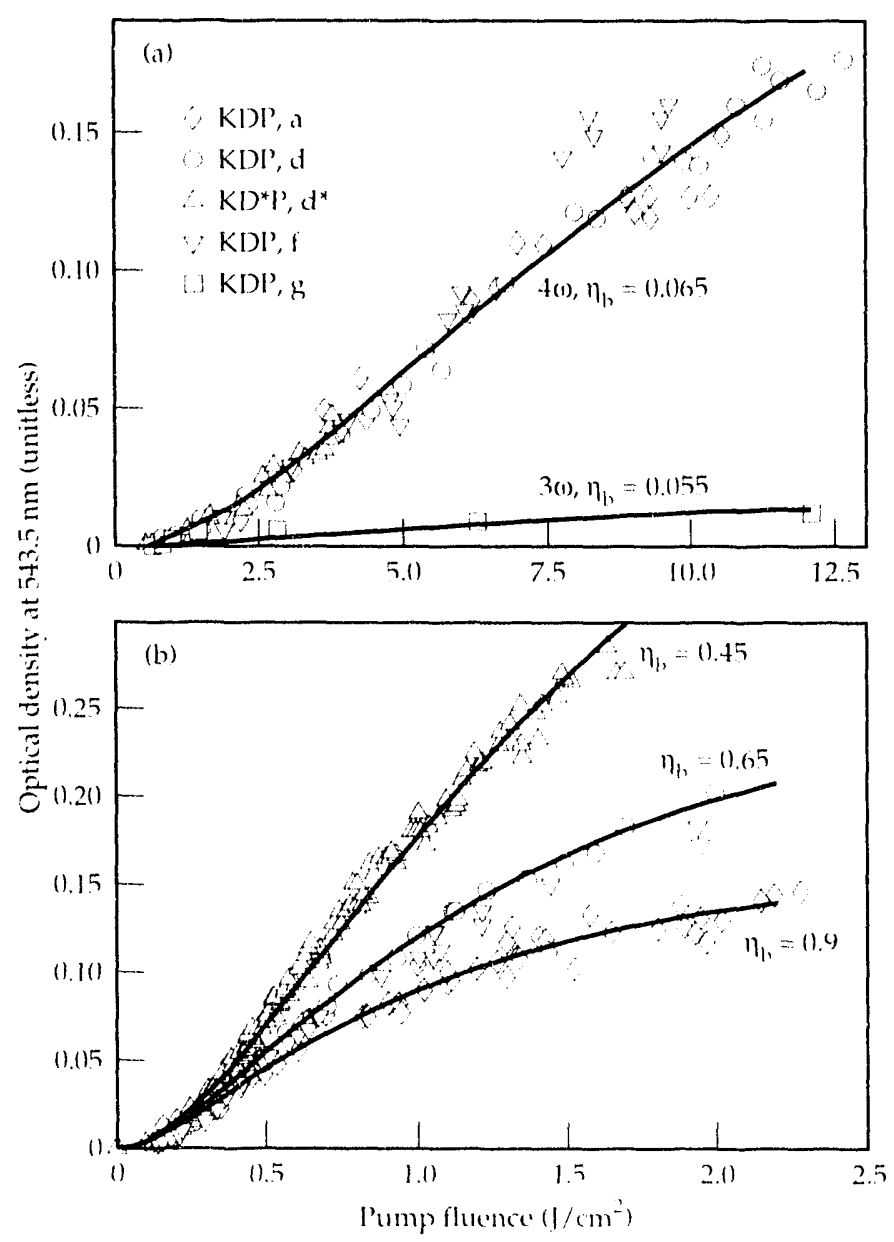

FICURI: 6. Fluence dependence of transient abourption strength at -1 ms delay times after excitation i, KDP and KD*P (rystals. (a) and (b) show data taken with - 10 ns and $~-100$ pes (iaussian pulses, respectively, using either $4(1)$ or. 3(1) excitation. The solid lines are fits to a model described by Eys. (2) - (5) and the parameters in Table 1. 
with $4 \omega$ pump light. This reflects the order of magnitude lower two-photon absorption constant at $3 \omega$ relative to $4 \omega .^{28,29}$

There is no evidence that the fluence-dependent absorption strength curves in Fig. 6 are related to the concentration of impurities discussed above, or to any change in the linear UV band-edge absorption due to impurities. In addition, the absorption strength is not correlated with the decay time of the transient absoiption. This implies that the transient absorption we observe is intrinsic to K.DP and is not substantially influenced by impurities. However, it would be useful to verify this assumption by conducting studies that change the dopant concentration in a controlled manner for elements, such as $\mathrm{Fe}$, that were below our detection limit.

\section{Physical Models}

With the information presented above, we postulate two physical descriptions of the defect state dynamics that describe the qualitative trends in the data. First, an electron is excited via two-photon absorption from the valence band into the conduction band of KDP. Subsequent interactions generate a defect state in the material with a formation efficiency of $\eta_{\mathrm{f}}$. For example, two photon absorption of $4 \omega$ radiation excites the tail of the oxygen-hydrogen antibonding orbital ${ }^{30}$ and may lead to the movement of a proton to a neighboring phosphate group. There is evidence in the literature ${ }^{12-18}$ that the defect state, $\mathrm{HPO}_{4}$-created by this process, in turn, absorbs light. Proton mobility has been previously studied, and the protons were found to have Arrhenius activation energies of $0.53-0.55$ for KDP and $0.53-0.58 \mathrm{eV}$ for $K D^{*} P^{31,32}$ This is in reasonable agreement with the measured defect decay activation energies of $0.51 \pm 0.04$ for all the KDP and $\mathrm{KD}^{*} \mathrm{P}$ crystals observed in our studies, which implies that the relaxation of the defect site, and hence its optical absorption, could be mediated by the diffusive recombination of a proton.

Alternatively, the electron promoted into the conduction band via two-photon absorption can relax and directly form an absorbing electronic center in the material at an absorbing impurity, dislocation, or vacancy without requiring protonic motion. This absorption is then bleached by optically exciting the electron back into the conduction band temporarily. The electron now either returns to the original nonabsorbing ground state (i.e. corresponding hole) or is trapped at a new dislocation or impurity, thus giving a bleaching efficiency of $\eta_{b}$. Once no additional light is incident on the sample, the defect state population can decay by thermally activating its bound electron to the conduction band and consequent recombination of the electron and corresponding hole.

\section{Numerical Modeling}

\section{Defect Formation}

We modeled the initial defect density that exists immediately after excitation by assuming that the defect is formed via two-photon absorption of 266-nm light and could then je bleached by subsequent $4 \omega$ irradiation. The three general equations governing the defect density $N$, pump intensity $I_{40}$, and probe intensity $I_{p}$ are expressed as

$$
\frac{d N(z, t)}{d t}=\frac{\eta_{f} \beta\left[I_{4 \omega}(z, t)\right]^{2}}{8 \hbar \omega}-\frac{N(z, t) \eta_{b} \sigma_{4(1)} I_{4(\omega)}(z, t)}{4 \hbar(\omega)},
$$

$$
\begin{aligned}
& \frac{d I_{4(1)}(z, t)}{d z}=-\left[\alpha_{4(1)}+N(z, t) \sigma_{4(1)}\right] I_{4(1)}(z, t)-\beta\left[I_{4(1)}(z, t)\right]^{2} \\
& \frac{d I_{p}(z, t)}{d z}=-\left[\alpha_{p}+N(z, t) \sigma_{p}\right] I_{p}(z, t),
\end{aligned}
$$

with an initial condition of

$$
N(z, t=-\infty)=0,
$$

where $z$ is the depth into the crystal, $\beta$ is the two-photon absorption constant, $\sigma$ is the defect absorption cross section, (1) is the Nd:YAG fundamental frequency, $\alpha_{4(1)}$ is the pump beam linear absorption constant, and $\alpha_{p}$ is the intrinsic probe absorption constant. In Eq. (2), the first and second terms describe defect formation via twophoton absorption and destruction or bleaching via linear absorption, respectively. The first term in brackets on the right hand side of Eqs. (3) and (4) is the linear absorption constant in the material; its two parts arise from the intrinsic linear and transient defect absorptions, respectively.

It is instructive to consider the most general case that allows for a closed form analytical solution to the coupled differential equations. In the absence of pump depletion, the z-dependence in Eq. (3) can be ignored and an uncoupled solution to Eq. (2) for the defect density $N(z=1, \tau)$ can be directly obtained for a temporally square pump pulse of length $\tau$ and intensity $I_{4(1)}^{0}$. The probe optical density OD measured at the output face of the crystal, $z=1$, can be obtained by multiplying $N(1, \tau)$ by both the appropriate probe absorption cross section $\sigma_{p}$ and the crystal length, 1. The probe optical density takes the form

$$
\begin{aligned}
& \text { Optical density }\left(\omega_{p}, t\right)=\frac{N(1, \tau) \sigma_{p}^{\prime}}{\ln (10)} \\
& =\frac{\eta_{f} \sigma_{p} \beta I_{4(1)}^{\prime}{ }^{\prime}}{\eta_{b} \sigma_{4(1)} 2 \ln (10)}\left[1-\exp \left(\frac{-\eta_{b} \sigma_{4(1)} I_{4(1)}^{(0)} \tau}{4 \hbar_{(1)}}\right)\right] .
\end{aligned}
$$


At low intensity, where the argument of the exponential is very small relative to unity, the probe optical density is proportional to the square of the $4 \omega$ intensity and is linear in the defect absorption cross section at $\omega_{\mathrm{p}}$. However, at high excitation fluence where the argument of the exponential is large relative to unity, the probe optical density at $\omega_{p}$ is linear in the pump intensity. In this region, the probe optical density is directly propoitional to $\eta_{f} / \eta_{b}$, and $\sigma_{p} / \sigma_{4 \omega}$. The limited solution given by $\mathrm{Eq}$. (6) qualitatively reproduces the quadratic and linear intensity trends also observed in the complete numerical solutions to Eqs. (2) - (4), as shown in Fig. 6(a).

To fit the experimentally observed probe optical density vs pump fluence curves shown in Fig. 6, we used the complete expressions given by Eqs. (2) - (5), including pump depletion. Gaussian temporal pulse shapes were used to model the two laser systems that were used as pump sources in the experimental section. The fits in Fig. 6 were numerically obtained by solving Eqs. (2) - (4) by finite differencing in both $z$ and $t$ and averaging the results over the Gaussian spatial excitation and probe pulse shapes in the $x-y$ dimensions. Table 1 presents the parameter values used to model the data. The $4 \omega$ twophoton absorption constant $\beta_{4(\omega)}$ used to fit the data in Fig. 6 was independently measured to be $0.06 \mathrm{~cm} / \mathrm{GW}$ at $266 \mathrm{~nm} .{ }^{33}$ These experiments used 100-ps pulses of a sufficiently low intensity that the transient absorption phenomena discussed in this paper does not play an important role. $0.06 \mathrm{~cm} / \mathrm{GW}$ is within the wide range of values ( 0.03 to $0.3 \mathrm{~cm} / \mathrm{GW}$ ) previously reported in the literature ${ }^{28,29}$ and well below the high values formerly required to account for the low $4 \omega$ frequency conversion efficiencies observed in the past at high $2 \omega$ drive intensity. $1,2,4-6$ The defect absorption cross section $\sigma$ used in the fits to Fig. 6 seems reasonable, since the corresponding oscillator strength (calculated using the peak cross section and spectral width) is $\sim 0.3$, which is consistent with typical oscillator strengths for color center absorptions.

The fits in Fig. 6(a) reproduce the qualitative trends expected from the limiting case solution given in Eq. (6). At low fluence, the probe optical density is quadratic in the fluence, becoming linear in the fluence as the fluence rises. As the fluence rises higher still, the curves become sublinear in the fluence, primarily due to pump depletion. At low fluence $\left(<0.5 \mathrm{~J} / \mathrm{cm}^{2}\right)$, the data is indistinguishable in both Fig. 6(a) and (b) for different samples, but above $0.5 \mathrm{~J} / \mathrm{cm}^{2}$ [Fig. $6(\mathrm{~b})$ ], the significant deviations between samples indicate that additional nonlinear processes may be present. This is particularly evident in Fig. 6(b), where the relatively short 100-ps pulse lengths lead to a maximum intensity of $20 \mathrm{GW} / \mathrm{cm}^{2}$ and direct two-photon absorption or depletion of the $4 \omega$ pump radiation is significant at about $50 \%$. Hence, we would expect a lower defect-induced optical density as a result

TABLE 1. Parameters used to model transient defect absorption strength and fourth harmonic conversion efficiencies.

\begin{tabular}{|c|c|c|c|}
\hline \multirow[b]{2}{*}{ Parameter (units) } & \multicolumn{3}{|c|}{ Numerical values used in } \\
\hline & $\begin{array}{l}\text { Induced absorption } \\
\text { experiment, Fig. } 6 \text { (a) }\end{array}$ & $\begin{array}{l}\text { Induced absorption } \\
\text { experiment, Fig. } 6 \text { (b) }\end{array}$ & $\begin{array}{l}4(1) \text { frequency } \\
\text { conversion } \\
\text { simulation, Fig. } 9\end{array}$ \\
\hline $\begin{array}{l}\text { Excitation pulse, Gaussian } \\
1 / \text { e full width (ns) }\end{array}$ & 10) & 0.1 & 1.2 \\
\hline $\begin{array}{l}\text { Two-photon absorption constant } \\
\beta(\mathrm{cm} / \mathrm{GW})\end{array}$ & $\begin{array}{l}0.06 \pm 0.03 \text { at } 40 \\
0.006 \pm 0.003 \text { at } .3(1)\end{array}$ & $0.06 \pm 0.03$ at $4(1)$ & 0.075 at 40 \\
\hline $\begin{array}{l}\text { Transient defect absorption } \\
\text { cross section } \sigma\left(\times 10^{-18} \mathrm{~cm}^{2}\right)\end{array}$ & $\begin{array}{l}4.6 \pm 2.3 \text { at } 2(1) \\
3.6 \pm 1.8 \text { at } 3(1) \\
2.7 \pm 1.3 \text { at } 4(1)\end{array}$ & $\begin{array}{l}4.6 \pm 2.3 \text { at } 2 \omega \\
2.7 \pm 1.3 \text { at } 4 \omega\end{array}$ & $\begin{array}{l}5.8 \text { at } 2(1) \\
3.4 \text { at } 4(1)\end{array}$ \\
\hline $\begin{array}{l}\text { Defect formation efficiency } \\
\eta_{f} \text { (unitless) }\end{array}$ & 1.0 & 1.0 & $\begin{array}{l}\text { 1.0) solid lines, } \\
\text { 0.() dashed lines }\end{array}$ \\
\hline $\begin{array}{l}\text { Defect bleaching efficiency } \\
\eta_{b} \text { (unitless) }\end{array}$ & $\begin{array}{l}0.065 \pm 0.04 \text { at } 4(1) \\
0.055 \pm 0.04 \text { at } 3(1)\end{array}$ & $\begin{array}{l}0.45,0.65, \text { and } 0.9 \\
\text { as noted in Fig. } 6\end{array}$ & 0.045 \\
\hline Linear absorption constant $\alpha\left(\mathrm{cm}^{-1}\right)$ & $\begin{array}{l}0.04 \text { at } 4(1) \\
0.02 \text { at } 30 \\
0.00 \text { at } 2(1)\end{array}$ & $\begin{array}{l}0.04 \text { at } 4(1) \\
0.00 \text { at } 2(1)\end{array}$ & $\begin{array}{l}0 .(04 \text { at } 4(1) \\
0 .(0) \text { at } 2(1)\end{array}$ \\
\hline Crystal thickness if $(\mathrm{cm})$ & 0.8 & 0.8 & 1.0 \\
\hline Angular detuning $\Delta \theta$ ( $\mu \mathrm{rad})$ & -- & - & 75 \\
\hline $\begin{array}{l}\text { Crystal face reflection losses } \\
\text { (\% per surface) }\end{array}$ & 4.0 & 4.0 & 4.0 \\
\hline
\end{tabular}


of the $4 \omega$ pump depletion. All para, neters were held constant between Fig. 6(a) and (b) except for $\eta_{b}$. For large $\eta_{b}$, the defect destruction or bleaching rate increases, which leads to a lower defect concentration and in turn a lower transient optical density.

It is not surprising that the effective $\eta_{b}$ used in Eq. (2) would be different for long and short pulses of equal intensity. In general, this optical bleaching process can take place many times during the initial UV excitation pulse. For a $4 \omega$ intensity of $0.5 \mathrm{GW} / \mathrm{cm}^{2}$, the average defect created during a 10-ns laser pulse excites and relaxes about $n=10$ times, as calculated from the relation

$$
n=\frac{(\tau / 2) I_{4 \omega} \sigma_{4 \omega}}{4 \hbar \omega}
$$

If the bleaching process were to arise from the promotion of the electron back to the conduction band followed by either recombination with the hole or subsequent retrapping, this would leave the sample, immediately after the propagation of the excitation pulse, with a random distribution of separations between the different defect species. On the other hand, occupied defect states created by the short 100-ps pulses are bleached only once or twice during the excitation pulse. Consequently, their $\eta_{b}$ is more closely correlated with the spatial concentration of unoccupied trap or defect sites, which can be highly variable between crystals.

\section{Defect Recombination}

The defect recombination or decay rate shown in Figs. 4 and 5 can be modeled using a theory originally developed to describe the annealing of crystalline defects in Ge induced by ionizing radiation. ${ }^{34}$ This theory assumes diffusive recombination of a pair of particles mediated by thermally activated diffusion. If the diffusive particles $\left(\mathrm{e}^{-}, \mathrm{H}^{+}\right.$, etc.) diffuse to within a radius $r_{0}$ of the corresponding recombination site, recombination takes place with an efficiency of unity. The initial probability distribution of separations between the two particles $\rho$ is assumed to be ${ }^{34}$

$$
\rho(r, t=0)=4 \pi r^{2} A \exp \left[-\left(\frac{r}{\lambda r_{0}}\right)^{2}\right]
$$

where $r$ is the radial separation between the defect and its corresponding pair, $A$ is a normalization constant such that the integral over all space is unity, and $\lambda$ is an adjustable parameter that sets the width of the distribution. We simplify the original equations ${ }^{34}$ by assuming that (1) only geminate recombination takes place and (2) the excitation intensity is low (i.e., there is no intensity dependence in the decay kinetics). In addition, we assume that $\lambda \gg>1$ (i.e., the initial distribution of defects following the excitation event is wide relative to the recombination capture radius $r_{0}$ ). Under these approximations, the equation governing diffusion-limited recombination becomes:

$$
\begin{aligned}
& \frac{N(t)}{N_{0}}=\frac{1}{\left[1+4 D t /\left(\lambda r_{0}^{2}\right)\right]^{1 / 2}} \\
& +\frac{1}{2(\pi \lambda)^{1 / 2}}\left[\arctan \left(\frac{4 D t}{\lambda r_{0}^{2}}\right)^{1 / 2}-\frac{\left[4 D t /\left(\lambda r_{0}^{2}\right)\right]^{1 / 2}}{1+4 D t /\left(\lambda r_{0}^{2}\right)}\right],
\end{aligned}
$$

where $N_{0}$ is determined by the initial concentration of absorbing defects. Using Eq. (9), the transient optical density in KDP in two representative samples as a function of time is shown in Fig. 7. The nonexponential

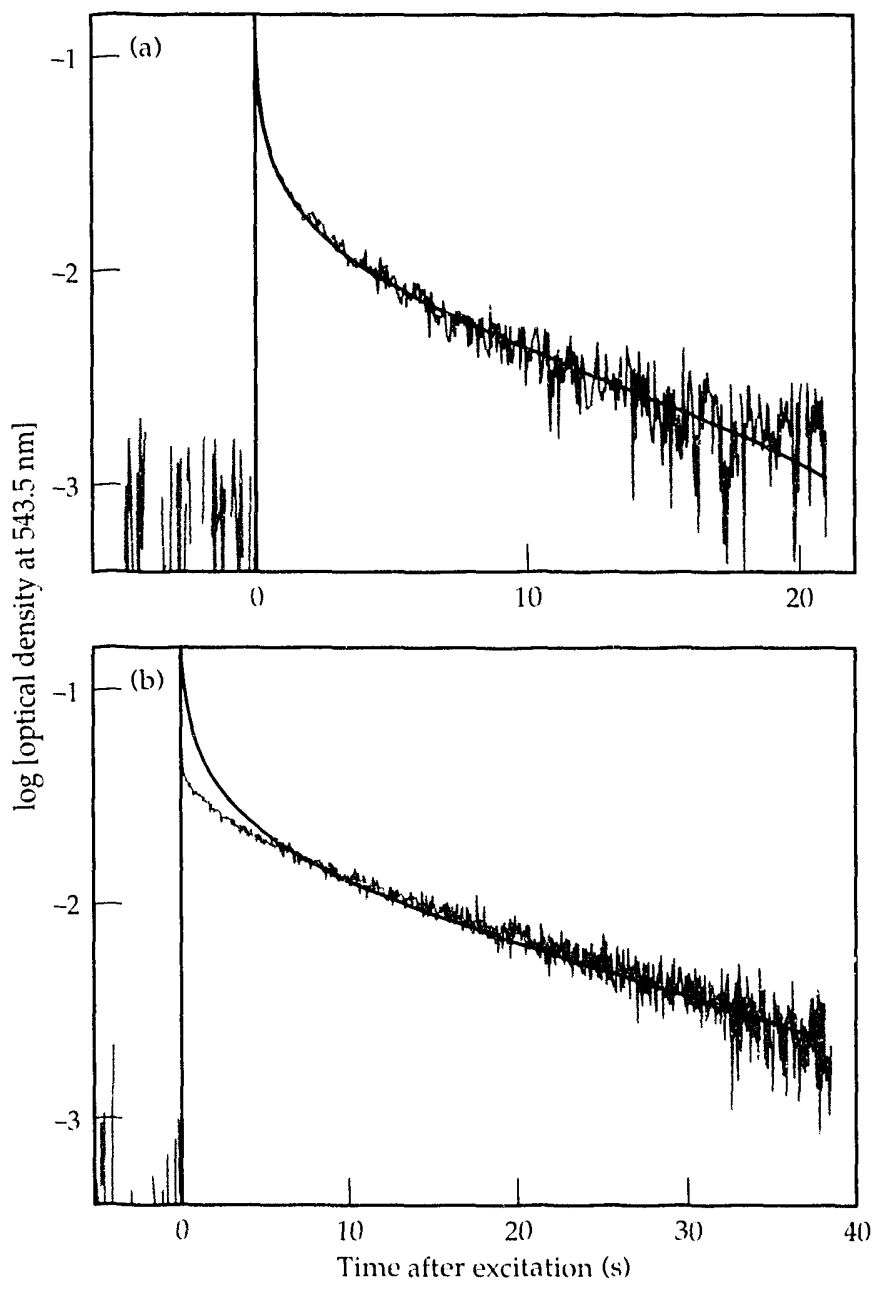

FIGURE 7. Time-dependent logarithmic plot of the transient absorption strengths for two representative samples. The nonexponential behavior can clearly be seen for all the samples studied. The fits described by $\mathrm{Eq}_{\mathrm{q}}$ (9) assume that a thermally activated diffusion-limited decay mechanism is present. 
behavior in both the fits and the data can clearly be seen between 0 and $5 \mathrm{~s}$ after excitation. Most of the decay data and fits taken on the fourteen KDP and $\mathrm{KD} * \mathrm{P}$ samples are in reasonable agreement as shown in Fig. 7(a), although about one third of the samples cannot be fit with any reasonable set of parameters as shown in Fig. 7 (b). It is probable that the assumptions made in either the model, such as the initial Gaussian distribution, or the approximations used in its solution are too simplistic. However, the basic assumption that there is a thermally activated diffusive recombination mechanism present seems reasonable.

\section{Multiple Pulse Excitation}

Figure 8 shows how the absorption strength saturates for multiple 10 -ns pulse excitations of sample $\mathrm{d}^{*}$ at $10 \mathrm{~Hz}$. Figures $8(\mathrm{a})$ and (b) were taken at single pulse fluences of 0.3 and $6.0 \mathrm{~J} / \mathrm{cm}^{2}$, respectively. Before zero time, there was no irradiation of the sample. After zero time, the laser irradiated the sample for 20 pulses. Following each

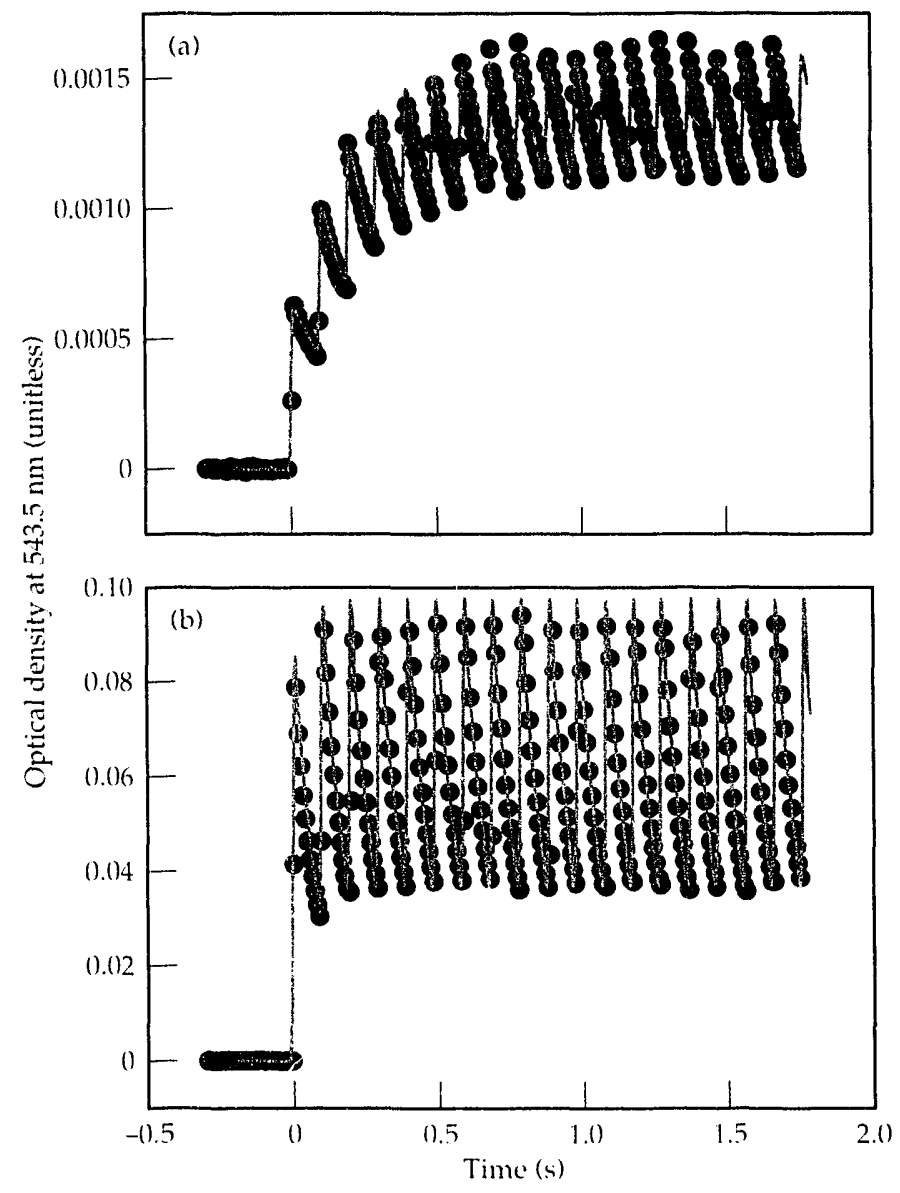

FICURE 8. Transient absorption strength in an $8 \mathrm{~mm}$ thick KD*P sample $\left(d^{*}\right)$ arising from successive $10-n$ s excitation pulses at 10$) \mathrm{ll}$ for (a) low (0.3 J/ $\left.\mathrm{cm}^{2}\right)$ and (b) high $\left(6 \mathrm{~J} / \mathrm{cm}^{2}\right)$ single-pulse fluences. irradiation, the sample absorption decayed at the same rate it would have for a single pulse excitation of the same fluence. The fits to the data were calculated using the numerical model described above with no adjustment of the absorption parameters (Table 1). The low fluence case saturates after $\sim 10$ pulses while the high fluence case saturates after 1-2 pulses. The data in Fig. 8 provides support for the fluence-dependent defect formation and bleaching model described in Eqs. (2)-(5), since the data and analysis show that the bleaching effect is cumulative over irradiation from multiple pulses. In addition, Fig. 8(b) indicates that pulsed high average power laser systems operating at $>0.5 \mathrm{GW} / \mathrm{cm}^{2}$ in the UV should not be substantially affected by transient absorption effects in KDP beyond that observed in single pulse applications because the transient absorption strength or optical density is virtually unchanged after the first pulse.

\section{Frequency Conversion to $4 \omega$}

Figure 9 compares predicted $2 \omega$ to $4 \omega$ frequency conversion efficiencies with experimental data taken on the Argus laser system. ${ }^{5,6} \mathrm{~A}$ time and space dependent numerical routine that includes linear, nonlinear, and transient defect-absorption at both $2 \omega$ and $4 \omega$, as well as crystal angle detuning and surface reflection losses was used to solve the nonlinear frequency conversion equations for KDP. ${ }^{11}$ The nonlinear coupling coefficients were taken from a recent experiment, ${ }^{4}$ while the other parameters were chosen to match the Argus experimental conditions.

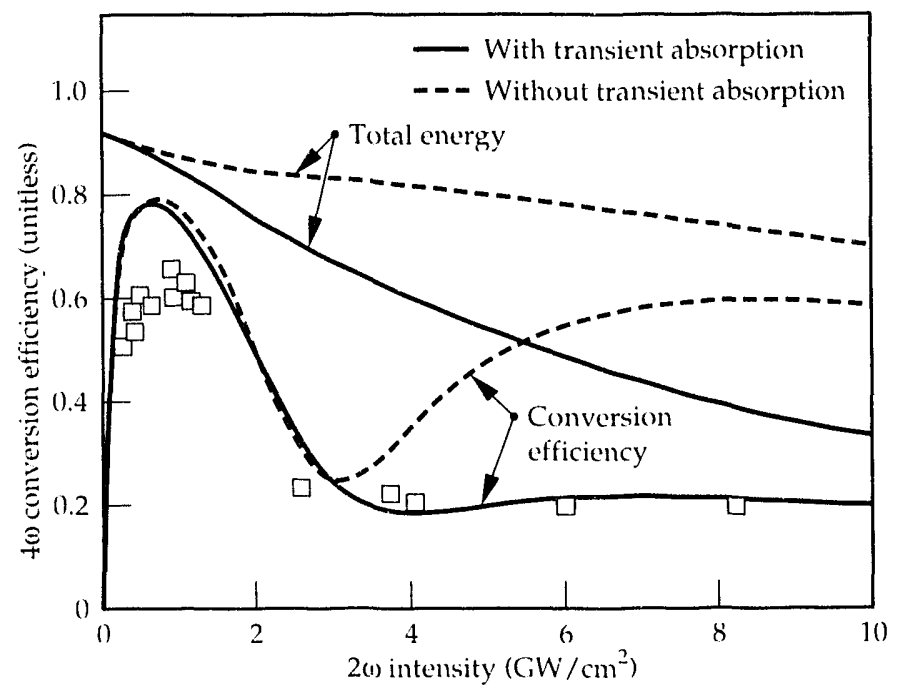

FIGURE 9. Calculated KDP frequency conversion efficiencies from 2(1) to 4 (1) plotted along with Argus data. ${ }^{5,6}$ The total normalized energy defined by the ratio of the light field energies incident on and exiting from the crystal are shown. The parameters used in the numerical simulations are defined in the last column of Table 1 . The solid and dashed lines represent calculations with and without the effects of transient absorption included in the simulations, respectively. 
The solid lines in Fig. 9 show a conversion efficiency calculation performed with the transient absorption parameters (Table 1). The measured transient absorption parameters were adjusted well within the error bars to obtain the fit. The dashed lines in Fig. 9 represent calculations that are identical to the solid lines except that the transient absorption effects have been eliminated by setting the defect formation efficiency to zero. In the absence of the transient absorption, the conversion efficiency oscillates, because of imperfect phase matching ${ }^{35}$, with moderate damping from twophoton absorption of the $4 \omega$ beam. This oscillation arises from competition between the forward and backward frequency conversion process. When transient absorption is included, the oscillation becomes overdamped primarily because the strong additional transient absorption at $2 \omega$ (Fig. 2) substantially reduces the forward-conversion driving field and the conversion efficiency never recovers from its initial drop. For the reported Argus experimental conditions $s^{5,6}$ with a $1-\mathrm{cm}$ crystal, comparisons of the theoretical peak $4 \omega$ conversion efficiency with and without the addition of transient absorption show it is reduced by only $\sim 2 \%$. Possible causes for the discrepancy between the experimental and theoretical peak conversion absolute magnitudes are: fill factor considerations from not aperturing the calorimeters, pump beam nonuniformities, imperfect beam collimation. Note that the effect of transient absorption on the maximum frequency conversion efficiencies for $3 \omega$ experiments should be small $(<1 \%)$ for similar drive intensities and pulse lengths since the two-photon absorption constant, and hence the defect concentration, is one order of magnitude smaller than at $4 \omega$. Even at $4 \omega$, only when the intensity is raised above $2 \mathrm{GW} / \mathrm{cm}^{2}$ does transient defect-absorption become a significant effect. It is clear in Fig. 9 that transient absorption from the UV-induced absorbing defects has a substantial impact on the $4 \omega$ conversion efficiencies at the larger drive intensities. The inclusion of transient defect absorption effects appears to explain, for the first time, the unusual flat shape and the lower than expected magnitude of the conversion efficiency curve at high drive intensities.

Since the transient absorption phenomena are essentially fluence driven rather than intensity driven, the temporal symmetry of the conversion process is broken and the initial portion of the laser pulse experiences a different absorption strength than the trailing side of the pulse. Our calculations show that transient absorption can lead to significant asymmetric temporal pulse shaping at both $2 \omega$ and $4(1)$, similar to that previously observed in $4 \omega$ frequency conversion experiments at high $2 \omega$ drive intensities. ${ }^{1,2}$ In addition, the transient absorption at $2 \omega$ can measurably affect the $4 \omega$ backward frequency conversion process. Consequently, with appropriate choices of crystal thicknesses and temporal pulse shapes, calculations show that it should be possible to use the induced transient absorption to reduce the backward conversion process and increase conversion efficiencies to the fourth harmonic. This is certainly an interesting prospect.

\section{Summary}

We have developed a physical model and a quantitative method to describe UV-induced broadband transient absorption in KDP and KD*P. The transient absorption was demonstrated to arise from UV twophoton absorption and subsequent relaxation to form a long-lived trapped defect site in the crystal. Our experimental measurements of transient absorption at 100-ps and 10-ns pulse lengths provided the parameter values required to successfully model the frequency conversion in KDP from 2(1) to $4 \omega$ with the inclusion of transient absorption. For the first time, favorable comparisons of $4 \omega$ conversion efficiencies were obtained between numerical simulations and the experimental data taken on the Argus laser system over a decade ago. 5,6 The new physical understanding of the fourth harmonic conversion process described in this paper should enable designers of frequency conversion systems to minimize the effects of transient absorption and maximize the final output $4(1)$ energy.

\section{Acknowledgments}

We would like to thank M. A. Henesian for his generous and energetic efforts in modifying his frequency conversion routine's to include transient absorption. In addition, we would like to thank C. Bibeau and $\mathrm{H}$. Powell for participating in many useful discussions and for providing their assistance and guidance throughout the course of this research.

\section{Notes and References}

1. I. H. Chaffee and R. B. Fhrlich," Small-Aperture, FourthHarmonic Comversion," laster Program Ammanal Repont, Lawrence Livermore National Laboratory, Livermore, CA, UCRL-50)21-87 (1987).

2. P. H. Chaffece, "Small-Aperture, t(1)-Comversion Exporiments," Laser logegrmm Ammmal Repurl, Law rence Livermore National Laboratory, Livermore, CA, UCRL-50)21-80 (1486).

3. K. R. Manes, (). C. Barr, E. S. Bliss, R. I. Drake, R. (). Codwin, D. C. Ciritton, J. S. Ifildum, F. W. I Jolloway, C. A. Iturley, B. C Johnson, D. J. Kuizenga, B. Merritt, R. (i. Oarski, F. R. Jr., J. R Severyn, D. R. Speck, M. A. Summers, (i. J. Suski, and E. I?. Wallerstein," Novette Facility: activation and experimental results," latice and Particle Be'ams, 3, (2) 17.3-188 (1985).

4. D. Bruncau, A. M. Tournade, and E. Fabre, "Fourth harmonic generation of a large-aperture Nd:glass laser," Appl. ()pt., 24, $3741)(1985)$. 
5. G. J. Linford, B. C. Hohnson, J. S. Hildum, W. E. Martin, K. Synder, R. D. Boyd, W. L. Smith, C. L. Vercimak, D. Eimerl, and J. T. Hunt, "Large aperture harmonic conversion experiments at Lawrence Livermore National Laboratory," Appl. Opt., 21, 3633 (1982).

6. G. J. Linford," Fourth-Harmonic Conversion-Efficiency Experiments," Laser Program Ammual Report, Lawrence Livermore National Laboratory, Livermore, CA, UCRL-5002181 (1981).

7. J. Reintjes and R. C. Eckardt, "Efficient harmonic generation from 532 to $266 \mathrm{~nm}$ in ADP and KD*P," Appl. Phy/s. Lett., 30, 91 (1977).

8. H. W. H. Lee, R. S. Hughes Jr. , L. L. Chase, and J. E. Davis, "Interaction of potassium dihydrogen phosphate with intense UV laser pulses: observation of defect formation," (1992 Tedinical Digest, Vol. 13, Optical Society of America, Washington, D. C., QThD10, 1992).

9. H. W. H. Lee, J. E. Davis and R. S. Hughes Jr., "Physics of optically generated transient electronic defect in $\mathrm{KH}_{2} \mathrm{PO}_{4}$ and $\mathrm{KD}_{2} \mathrm{PO}_{4}, " \mathrm{Nonlintear}$ Optics: Materials, Fundamentals, and Applications Conference, Lahaina, HI, (1992).

10. J. E. Davis, R. S. Hughes Jr. and H. W. H. Lee, "Investigation of optically generated transient electronic defects and protonic transport in hydrogen-bonded molecular solids: Isomorphs of potassium dihydrogen phosphate," Ch'm. Phy/s. Lett., 207, 540 (1993).

11. M. A. Henesian, Time-dependent frequency conversion code, Lawrence Livermore National Laboratory, Livermore, CA, 1993.

12. E. Dieguez and J. M. Cabrera, "Optical absorption and luminescence induced by $\mathrm{X}$-rays in KDP, DKDP, and ADP," I. Ch'm. Physs, 81, 3369 (1984).

13. E. Dieguez and J. M. Cabrera, "Optical absorption and thermoluminescence of X-irradiated KDP," I. of Plyysics D (Applict Plyysics), 14, 91 (1981).

14. J. A. McMillan and J. M. Clemens, "Paramagnetic and optical studies of radiation damage centers in $\mathrm{K}\left(\mathrm{H}_{1-x} \mathrm{D}_{x}\right)_{2} \mathrm{PO}_{4}$, " I. Ch'm. Phys., 68, 3627 (1978).

15. A. I. Ryabov, N. S. Stelmakh, G. N. Pirogova, Y. Voronin, and B. I. Zakharkin, "Radiation-induced coloring of nonlinear crystals at low temperatures," Sor. Physics Sol. State, 33, 2660 (1991).

16. G. N. Pirogova, Y. V. Voronin, V. E. Kritskaya, A. I. Ryabov, and N. A. Malov, "Effect of radiation the optical properties of some ferroelectrics," Izv. Akad. Nouk SSSR, Neorg. Mater. (lnorgmic Materials), 22, 97 (1986).

17. H. I. Farag, M. S. Elmanharawy, and A. Abdel-Kader, "Some temperature dependent properties of potassium dihydrogen phosphate," Acta. Physicn Hungarica, 60, 19 (1986).

18. V. V. Azarov, B. P. Gritsendo, V. M. Lisitsyn, and V. I. Salo, "Decay Time of Short-Lived Radiation Color Centers in DKDP Single

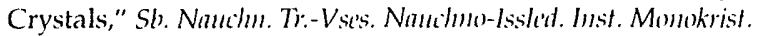
Stsintill. Mater. Osobo Chist. Khim. Veshche'str' 12, 34-36, (1986).

19. J. J. DeYoreo, Lawrence Livermore National Laboratory, Livermore, CA, private communication, (1992).
20. B. V. Andreev, Y. N. Kostrubov, L. G. Karaseva, and V. V. Gromov, "Formation of radiation defects in $\mathrm{KH}_{2} \mathrm{PO}_{4}$ : temperature dependence of the dose-rate effect," I. Phys. Condens. Matter, 1, 3359 (1989).

21. A. N. Levchenko, V. M. Shulga, and A. O. Doroshenko, "Impurity origin of the optical absorption and luminescence spectra of irradiated $\mathrm{KH}_{2} \mathrm{PO}_{4}$ crystals," Sor. Phys. Solid State, 32, 1432 (1990).

22. J. K. Rath and S. Radhakrishna, "Optical properties of $\mathrm{SeO}_{4}{ }^{3-}$ Centers in $\mathrm{NH}_{4} \mathrm{H}_{2} \mathrm{PO}_{4}$ crystals," Solit Statc Commun., 65, 1621 (1988).

23. G. N. Pirogova, Y. Voronin, A. I. Ryabov, and N. A. Malov, "The effect of temperature on the generation of radiation defects in nonlinear-optical crystals," Izv. Akad. Nuak SSSR, Neorg. Mater.. 23, 477 (1987).

24. E. Dieguez, A. Cintas, P. Hernandez, and J. M. Cabrera, "Ultraviolet absorption and growth bands in KDP," I. of Crystal Growth, 73, 193 (1985).

25. B. V. R. Chowdari and Y. R. Sekhar, "Radiation damage of $\mathrm{CrO}_{4}{ }^{2-}$ doped $\mathrm{KH}_{2} \mathrm{PO}_{4}$ single crystals," |. Ch'm. Phys., 75, 2513 (1981).

26. O. M. Arbotoev, A. A. Alybakov, and T. F. Veremeichik, "Identification of the valency of chromium ions in single crystal: of alkali-metal and ammonium phosphates from optical spectra," I. Appliced Spectroscopy, 55, 924 (1991).

27. I. Fujita, T. Suzuki, and Y. Miwa, "Determination of oscillator strengths for the Tl-centers in potassium dihydrogen phosphate single crystals," I. Physical Soc. of Japan, 51, 3998 (1982).

28. J. Reintjes and R. C. Eckhardt, "Two-photon absorption in ADP and KD*P at $266.1 \mathrm{~nm}$," IEEE I. Qumitum Ele'., QE-13, 791 (1977).

29. P. Liu, W. L. Smith, H. Lotem, J. H. Bechtel, N. Bloembergen, and R. S. Adhav, "Absolute two-photon absorption coefficients at 355 and $266 \mathrm{~nm}, "$ Phys. Re'? $B, 17,4620$ (1978).

30. S. Saito and R. Onaka, "Electronic structures of KDP and its family," Ferrolectrics, 21, 553 (1978).

31. V. H. Schmidt and E. A. Uehling, "Random motion of deuterons in $\mathrm{KD}_{2} \mathrm{PO}_{4}$," Plyys. Re'?, 126, 447 (1962).

32. L. B. Harris and G. J. Vella, "Direct current conduction in ammonium and potassium dihydrogen phosphate," 1 . Ch'm. Phys., 58, 4550 (1973).

33. H.W. H. Lee and R. S. Hughes, "Antiresonant ring in measuring nonlinear absorption," Conferencie on Lasers and Electro-Optics, (1992 Technical Digest, Vol. 12, Optical Society of America, Washington, D. C., CTuK2, 1992).

34. T. R. Waite, "Diffusion-limited annealing of radiation damage in Germanium," Plyys. Rez., 107, 471 (1957).

35. J. A. Armstrong, N. Bloembergen, J. Ducuing, and P. S. Pershan, "Interactions between light waves in a nonlinear dielectric," Phys. Re'?, 127, 1918 (1962). 


\title{
ReLATIVISTIC SeMiclassical Atomic Transition Rates
}

\author{
R. M. More
}

\section{Introduction}

Computer codes for the simulation of inertial fusion require atomic data in order to represent the generation and absorption of $x$ rays by highly charged ions of the target materials. In recent years we have explored semiclassical methods for calculation of this data: this approach seems to be the only systematic way to move from the fundamental theory to closed-form expressions for atomic transition rates.

We found simple algorithms ${ }^{1,2}$ for calculation of radiative rates (for bound-bound and bound-free transitions) that give good answers for hydrogenic and nonhydrogenic ions. These have been extended to include the effects of the dense plasma environment and again give satisfactory answers. A new semiclassical screening model, partly suggested by the work of European researchers, ${ }^{3}$ appears to give reasonably accurate energy levels and wave functions for arbitrary high-charge ions without loss of the simple algebraic form of the semiclassical equations. This work is based on nonreliativistic semiclassical theory, but relativity has large effects in high- $Z$ atoms such as gold. Inner-shell electrons have velocities comparable to the speed of light, and this modifies their $x$-ray spectra. Core levels are shifted to higher energies and split by the spin-orbit interaction to produce fine-structure multiplets. Relativity also changes the matrix elements that control $x$-ray line intensities.

We have now developed a relativistic semiclassical calculation of radiative rates based on a new WKB solution of the Dirac equation for an electron in a spherically symmetric atomic potential $U(r)$. The wave function is simple enough for rapid numerical evaluation, and the results are accurate enough for laser-plasma applications. The new solution exactly reduces to the nonrelativistic WKB solution in the low-Z (or low-velocity) limit, and is therefore compatible with our previous work.

\section{New WKB Wave Functions}

Generalizing the usual WKB approximation, we write the two-component wave function of an electron as

$$
\psi(r)=\left[\begin{array}{l}
F(r) \\
G(r)
\end{array}\right] \exp \left[i \int q(r) d r\right]
$$

The prefactor functions $F(r), G(r)$ vary slowly with radius $r$; the exponential function varies rapidly. An equation for the wave vector $q(r)$ is given below.

We require that the function $\psi$ solve the Dirac equation to the accuracy of the rapidly varying exponential functions. This gives the explicit WKB solution:

$$
\begin{aligned}
& F(r)=N_{0} \sqrt{\frac{m c^{2}+E-U(r)}{2 m c^{2} q(r)}}, \\
& G(r)=\sqrt{\frac{E-U(r)+m c^{2}}{E-U(r)+m c^{2}}} \sqrt{\frac{\kappa / r+w(r)+i q(r)}{\kappa / r+w(r)-i q(r)}} F(r) .
\end{aligned}
$$

Here, $N_{0}$ is a normalization constant, $E$ is the total electron energy including the rest energy $m c^{2}, U(r)$ is the electron-nucleus potential energy, and $\kappa= \pm(j+1 / 2)$ is a relativistic angular momentum parameter used to label the states, where $j$ is the total angular momentum quantum number. In Eqs. (2) and (3), the function $z(r)$ is a relativistic contribution to the centrifugal potential, determined by

$$
\frac{d}{d r} \log \left[\frac{\kappa / r+w(r)}{E-U(r)+m c^{2}}\right]=-2 w(r) .
$$


This equation can easily be solved for $w(r)$ if we know the potential $U(r)$.

The first square-root factor in Eq. (3) is a magnitude factor: it shows that $G$ is smaller than $F$ by a ratio proportional to $v / c$. Thus $G(r)$ vanishes in the nonrelativistic limit. $F(r)$ will approach the nonrelativistic WKB wave function if the momentum $p(r)=\hbar q(r)$ approaches the proper nonrelativistic limit.

The radial momentum $p(r)$ is determined by

$$
[E-U(r)]^{2}=\left(m c^{2}\right)^{2}+c^{2} p^{2}+\hbar^{2} c^{2}\left[\frac{\kappa}{r}+w(r)\right]^{2} .
$$

Equation (5) can be understood by comparison to the relativistic relation between mass and energy for a free electron moving in three dimensions with vector momentum p:

$$
E^{2}=\left(m c^{2}\right)^{2}+c^{2} \mathbf{p}^{2}
$$

Our Eq. (5) is very similar; however, the total energy $E$ is reduced by subtracting the potential energy $U(r)$ and the kinetic energy is expressed as the sum of a radial contribution $\left(c^{2} p^{2}\right)$ and a part associated with the angular motion. This angular term includes the centrifugal kinetic energy and the spin-orbit interaction.

We can test this relativistic wave function by forming the probability density, which turns out to be

$$
\psi^{\dagger} \psi=\frac{\text { constant }}{v_{r}(r)}
$$

Here $v$ is the relativistic velocity, which cannot exceed the speed of light. Equation (7) is the natural generalization of a nonrelativistic result and shows that the probability density is highest where the electron moves slowly.

TABLE 1. Percentage changes in the expectation value of radius $\langle r\rangle$ caused by relativity for various levels of hydrogen-like gold. This comparison shows how accurately the relativistic semiclassical theory would place the electron charge-density in a many-electron selfconsistent-field calculation.

\begin{tabular}{lll} 
State & Exact & Semiclassical \\
\hline $1 s_{1 / 2}$ & -12.2 & -17.5 \\
$2 s_{1 / 2}$ & -13.0 & -13.9 \\
$2 p_{1 / 2}$ & -15.6 & -15.5 \\
$2 p_{3 / 2}$ & -03.4 & -04.2 \\
$3 s_{1 / 2}$ & -09.9 & -10.4 \\
$3 p_{1 / 2}$ & -10.7 & -10.7 \\
$3 p_{3 / 2}$ & -03.7 & -03.9 \\
$3 d_{3 / 2}$ & -04.5 & -04.3 \\
$3 d_{5 / 2}$ & -01.6 & -01.9 \\
\hline
\end{tabular}

Equation (7) can be read as a conservation law $\left(v_{\mathrm{r}}|\psi|^{2}=\right.$ const $)$, which expresses the conservation of probability current. Our approximate solution exactly satisfies this important test.

The centrifugal energy can be expanded for low- $Z$ ions

$$
\hbar^{2}\left(\frac{\kappa}{r}+w\right)^{2}=\frac{\hbar^{2}(l+1 / 2)^{2}}{r^{2}}-\frac{Z e^{2}(l+1 / 2)^{2}}{2 \kappa m c^{2}} \frac{1}{r^{3}}+\ldots .
$$

The first term is the nonrelativistic centrifugal energy, and, when evaluated with the semiclassical average $\left\langle 1 / r^{3}\right\rangle$, the second term is exactly the spin-orbit interaction energy (including the Thomas precession). Therefore the relativistic semiclassical theory gives the correct energy it vels to order $Z^{4} \alpha^{4} m c^{2}$ (where $\alpha \approx$ $1 / 137$ is the fine-str ucture constant).

With these results we see that the radial momentum $p=\hbar q$ reduces (for low-velocity electrons) to the correct nonrelativistic semiclassical limit in all details including the use of $l+1 / 2$ in the centrifugal energy. Then the entire theory behaves properly in the nonrelativistic limit.

Thus, the main formal advantages of our solution are that it exactly obeys probability conservation and that it exactly reduces to the nonrelativistic semiclassical theory. It is also important that our solution is simple to work with.

\section{Quantization and the Energy Levels}

The semiclassical quantization condition is

$$
\oint q(r) d r=2 \pi\left(n_{\mathrm{r}}+\frac{1}{2}\right) .
$$

This equation serves to determine the energy eigenvalue $E_{n l i}$. The radial quantum number $n_{\mathrm{r}}$ is an integer.

The electron energy has the form obtained by Sommerfeld:

$$
E_{n l j}=\frac{m c^{2}}{\sqrt{1+Z^{2} \alpha^{2} / N^{2}}},
$$

where

$$
N=\left(n_{\mathrm{r}}+\frac{1}{2}\right) \pm \frac{1}{2}+\Delta+\backslash \kappa^{2}-Z^{2} \alpha^{2}
$$

Here the \pm sign agrees with the sign of $\kappa$, and $\Delta$ is a small term present only in the semiclassical theory.

We have computed semiclassical energy levels for many cases. Because of the term $\Delta$ in Eq. (11), the energies differ slightly from the (exact) Sommerfeld energy 
levels for hydrogenic ions, but these differences are never more than $1 / 2 \%$ and will not be important for most applications. The differences are very small for large quantum numbers, and when the energy is expanded in powers of $v / c$ or $Z \alpha$, the first three terms of the series are exactly correct.

Table 1 gives changes caused by relativity in the average orbit radius for an electron in hydrogen-like gold. The semiclassical results are quite accurate except for the lowest state, and the changes depend mainly on the quantum number $j$ rather than on $l$. These changes are mainly caused by spin-orbit interaction; in effect, the moving electron is like a small bar magnet in an inhomogeneous magnetic field (the Lorentz transform of the nuclear electric field) and is attracted or repelled depending on its orientation. This spin-orbit effect combines with the overall contraction of the orbit produced by the relativistic mass increase to give the results in Table 1.

Because the semiclassical wave functions have reasonably accurate radial position, as shown by Table 1 , we expect that the theory will give a good basis for rapid self-consistent field calculations of many-electron ions.

\section{Matrix Elements}

As a test of the relativistic WKB wave function, we calculate dipole matrix elements

$$
R_{n l l i}^{n^{\prime} l^{\prime} i^{\prime}}=\int_{0}^{(1)}\left(F_{n l j}^{*} r F_{n l^{\prime} l^{\prime} i^{\prime}}+G_{n, j^{\prime}}^{*} r G_{n l^{\prime} l^{\prime} i^{\prime}}\right) e^{i \Delta \phi} d r \text {, }
$$

where the exponential factor is the difference of phase integrals $\int_{q}(r) d r$ for initial and final states. The integral is evaluated by a saddle-point method. This formula

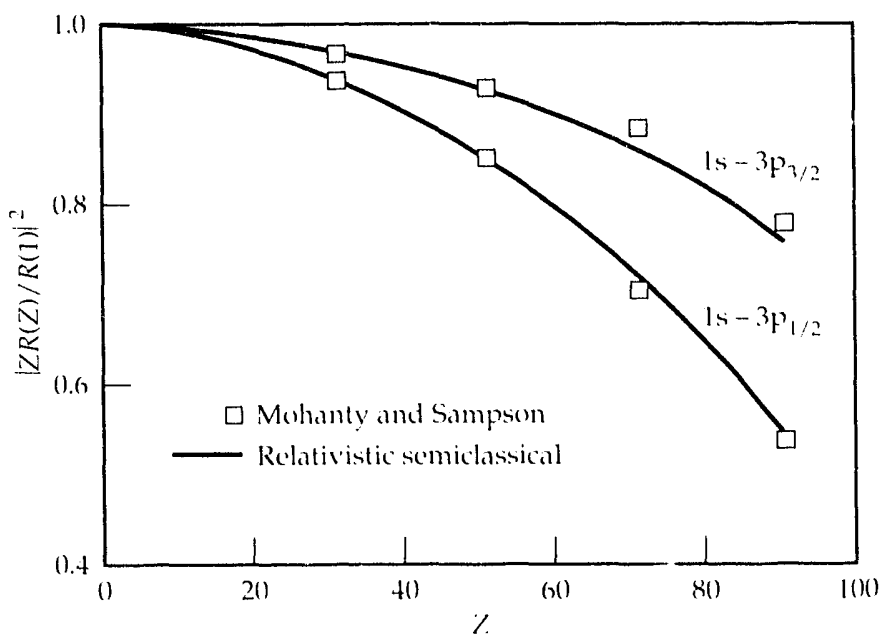

FIC iURE: 1. Effect of relativity on radiative rates for $15 \rightarrow 3$ p transitions for hydrogen-like ions of nuclear charge 7 . calculated by our methed and by exact solution of the Dirac eyuation. Relativity strongly reduces the rate for this transition. The quantity plotted is $\mid k R(C) / R(1) F^{2}$, where $R(\%)$ is the relativistic dipole moment and $R(1)$ is the dipole moment for this transition in hydrogen $(\%=1)$. and our comparison data omit the retardation correction, so the comparison is mainly a test of the relativistic WKB wave functions.

Figures $1-5$ show some results. The quantity plotted is the dimensionless ratio $|Z R(Z) / R(1)|^{2}$. Because the nonrelativistic hydrogenic matrix elements are exactly proportional to $1 / Z$, this ratio would be unity except for the effects of relativity.

The graphs show that relativity has a large effect, especially for transitions with small angular momentum. The semiclassical calculations follow these changes even for the unusual cases in which changes in the matrix element are especially small or especially large.

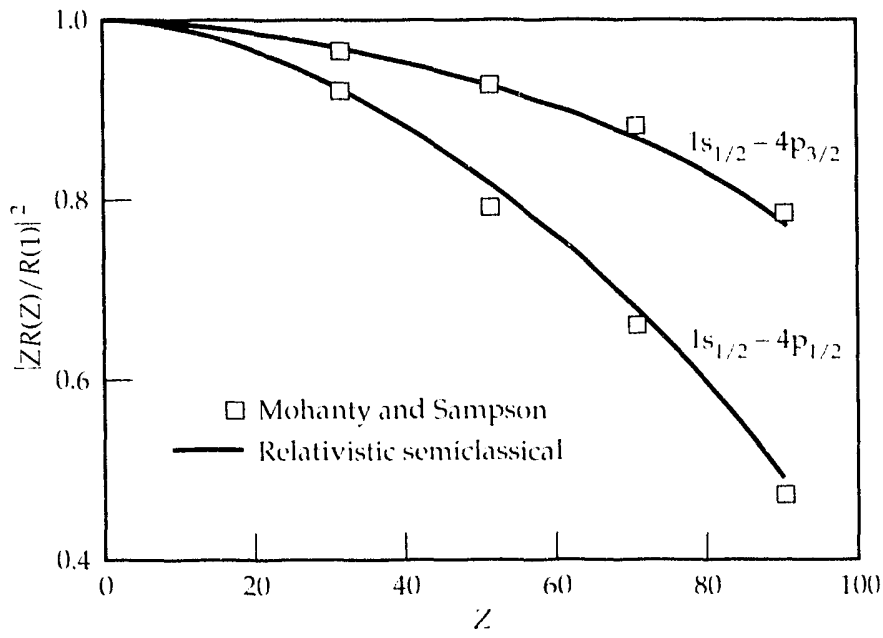

FICURRE 2. Effect of relativity on radiative rates for $1 s \rightarrow 4 p$ transitions.

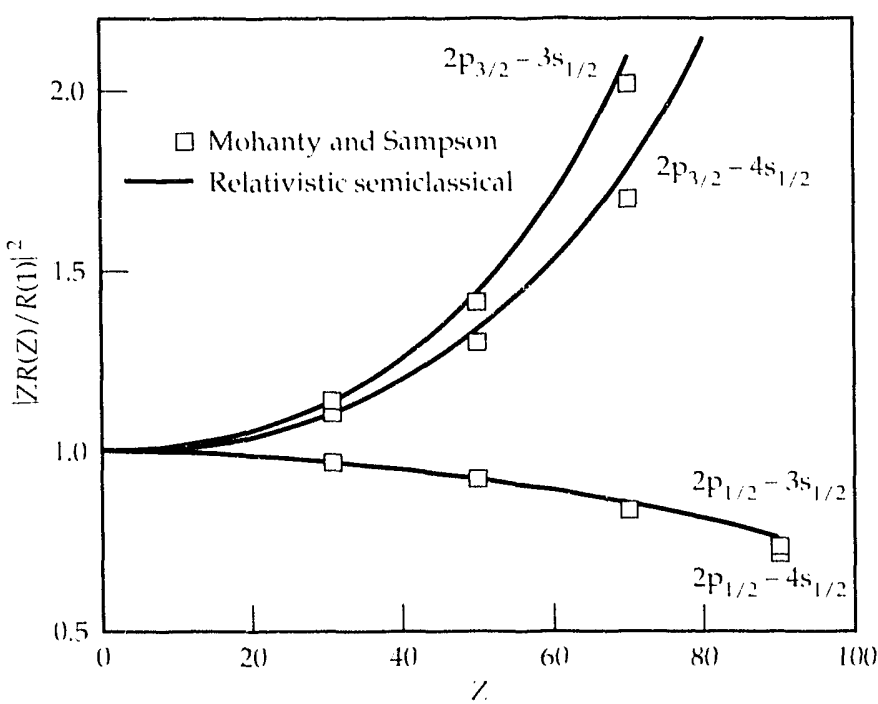

FliURE 3 . Leffect of rehativity on radiative rates for $2 p \rightarrow 3$ and $2 \mu \rightarrow$ ts transitions. Some rates are strongly increased by relativity. 


\section{Summary}

We have described a semiclassical (WKB) treatment of relativistic one-electron effects in atomic structure. The work is based on a new approximate solution of the Dirac equation. ${ }^{7}$

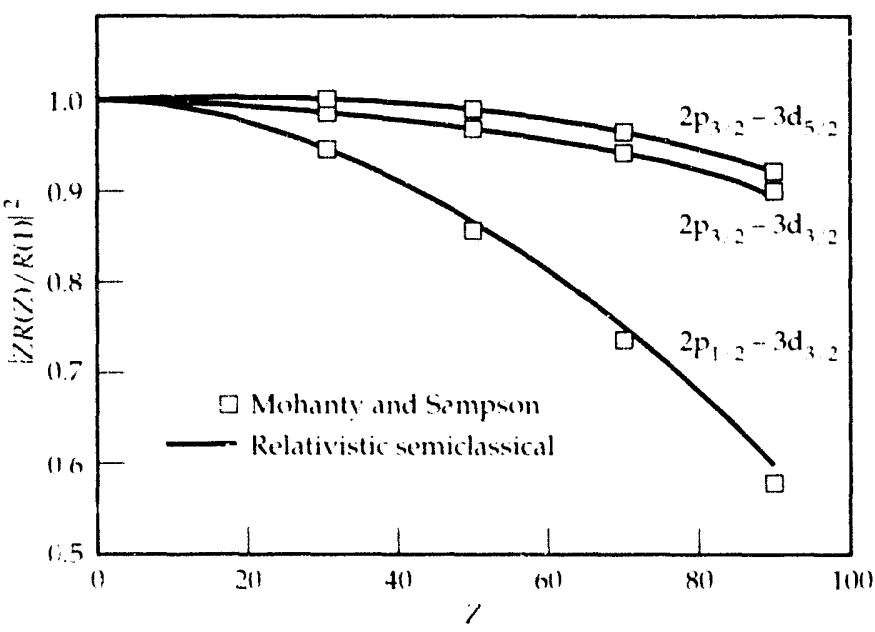

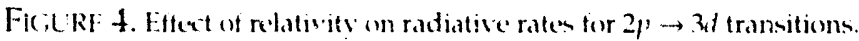

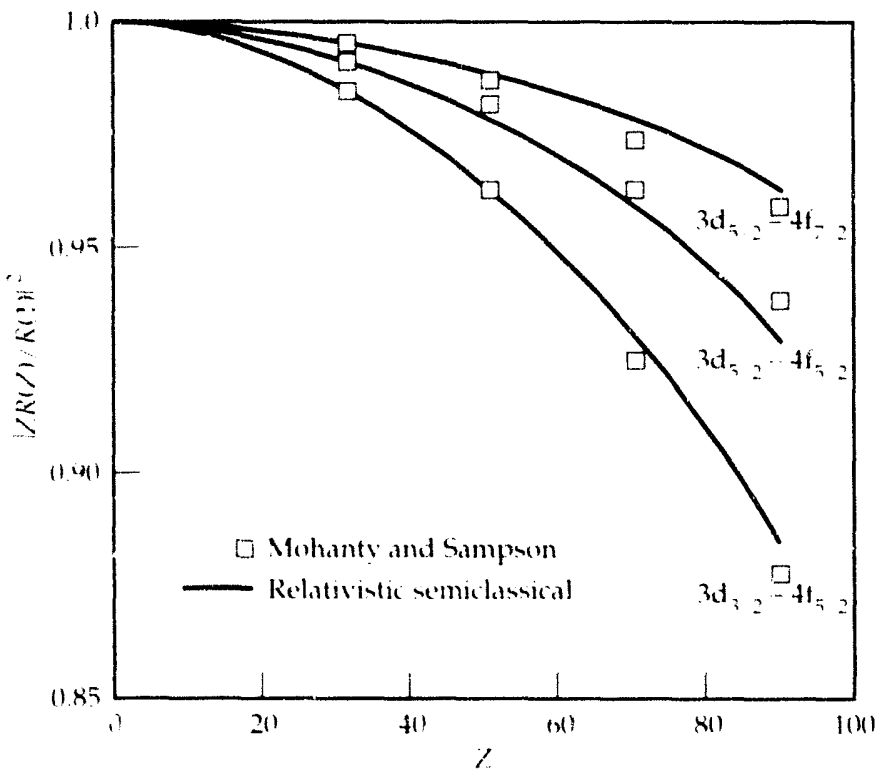

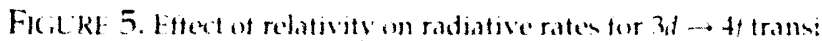
tion- Here the effect of relatiosty is quite amall (netice the expanded

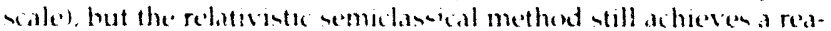
unatily high accursos
The solution is well behaved for large and small quantum numbers. It obeys a relativistic probabilityconservation theorem [Eq. (7)], and has a generalized centrifugal energy that includes the spin-orbit interaction energy.

The energy levels, wave functions, and matrix elements exactly reduce to tile nonrelativistic semiclassical quantities in the appropriate limit (low-velocity electrons having large angular momentum, or inner electrons of low- $Z$ ions).

The wave function has the analytic properties required for the calculation of matrix elements by contour integration. The results obtained are reasonably accurate.

We believe that trese methods can be extended to provide a LASNEX subroutine that will give much better treatment of radiative processes important to ICF targets and to laser experiments in general.

\section{Notes and References}

1. R. M. More and K. H. Warren, "Semiclassical Calculation of (Bscillator Strengths," I. Phys. (l'aris) 50, 35-44 (1489).

2. R. M. More and K. H. Warren, "Semiclassical Calculation of Matri Elements," Ann. Physs. 207, 282 (1991); R. M. More, "Semiclassical Matrix Mechanics 2: Angular Momentum Operators," I. Phys. 11 (2), 97-121 (1991).

3. i'. Pankratow and 1. Meyer-ter-Vehn, "Semiclassical Energy" Levels and the Corresponding l'otentials in Nonhydrogenic fons," Pins. Rer. A 46 (4), 5497-5499 (1992).

4. R. M. More, "Electronic Energy Lenels in Dense Plasmas," 1. (Jumt. Sf ctrinsi Radiat. Trumsite 27 (3), 345-357 (1482)

5. A. K. Mohanty and D. H. Sampson, "Reply to 'A Comparison of Relativistic and Quasirelativistic Line Strengths'," Physica Siripta 38, 557-554 (1988).

6. S. J. Ruse. "The Calculation of the Opacity of Ilot Dense" Plasmas," Rutherford-Appleton Laboratory, Chiltom, Didoot, ()on. England, RAL_-85-1)22 (March 1985); "The Effect of Relatwivi on the Oncillator Strengths of Hydrogen-like lons," Rutherford-Appleton Laboratory, Chilton, Didcot, (Oxon, England, RL-82-114 (December 1482).

7. R. M. More, "Relativintic Semiclassical Atomic Transition Rates," LCRL-J - 113714 (1943), submitted for publication in

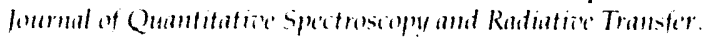




\title{
VERIFICATION OF OPAL OPACITY CODE PREDICTIONS FOR CONDITIONS OF ASTROPHYSICAL INTEREST
}

\author{
R. W. Lee \\ C. A. Iglesias \\ L. B. Da Silva
}

\section{Introduction}

Although radiative transport is essential in determining the structure and evolution of stars, there have been no direct measurements of radiative opacity at conditions characteristic of stellar interiors. Over the past several decades, stellar models have therefore relied on opacities obiained from theoretical calculations, mostly those carried out at Los Alamos National Laboratory. ${ }^{1}$ Although these opacities lead to a correct description of the main features of stellar evolution, many details remained unexplained. Recently, the situation has been altered with the introduction of new calculations with the OPAL opacity code ${ }^{2}$ that show much higher opacities than the Los Alamos results. These new opacities have led to the resolution of several long-standing puzzles in stellar pulsation properties of Cepheids, $\delta$ Scutis, and $\beta$ Cephei stars, ${ }^{3}$ and have made a favorable impact on solar r.mudels, ${ }^{4}$ the critical mass limit for pulsationally stable stars, ${ }^{5}$ convective core overshoot," and lithium depletion in the Hyades. ${ }^{7}$ Still, these successes represent only indirect validations of the new opacities. Recent advances in experiments with laser-produced plasmas permit measurements of photon absorption for conditions relevant to stellar envelopes, thus providing direct tests of opacity models.

\section{OPAL Opacity Code}

OI:AL ${ }^{2}$ contains two basic improvement over older calculations. First, a parametric potential approach has been developed that is computationally fast and that yiclds photon absorption cross sections whose accuracies are comparable to those obtained in single-configuration, self-consistent-field calculations with relativistic corrections." These potentials therefore permit the calculation of large quantisies of reasonably accurate atomic data for elements with low to medium atomic number $(Z \leq 35)$. In calculating opacities, OPAL includes, for the first time, the detailed configuration term structure, which is treated in LS or intermediate coupling, ${ }^{10}$ depending on atomic number. The efficient computation of atomic data makes it possible to generate tables of the Rosseland mean opacity for use in astrophysical models ${ }^{2}$ and to generate tables of frequency-dependent absorption coefficients for use in large hydrodynamic simulations with codes such as LASNEX.

A second, more subtle improvement in OPAL is in the equation of state, which is obtained through systematic expansions of the grand canonical partition function. ${ }^{11}$ The method avoids the usual nit hoc cutoffs necessary to eliminate the divergerice of the internal partition function in free-energy-minimization approaches. No cutoff is required in the activity-expansion method, because performing un complete trace over both bound and scattering states removes the divergence. " The lowestorder term in the activity expansion (which includes Coulomb corrections) is the well-known Debye-Hückel term, but higher-order terms are also retained.

The modifications in the equation of state lead to moderate opacity increases for the solar radiative interior ${ }^{2}$; the improvements in the atomic physics lead to large opacity increases for temperatures of a few hundred thousand kelvins. In this temperature range, typical of stellar envelope conditions and critical for pulsation models, the configuration term structure in iron leads to many more spectral lines than calculations that neglect the term splitting. ${ }^{2,13}$ The experimental confirmation of the new OPAL results therefore emphasizes the single most important modification of the opacity, which arises from bound-bound transitions in iron for $n=3$; that is, $\Delta n=()$ transitions of ions with partially filled M-shells. 


\section{Experiment}

The experiment uses a variation of techniques developed for photon-absorption measurements at the Nova laser facility. ${ }^{14}$ Figure 1 shows the arrangement. One laser beam, with a wavelength of $0.53 \mu \mathrm{m}$, is used to heat a $2500-\AA \mathrm{Au}$ foil by direct irradiation with a $3.3-\mathrm{kJ}$, 1 -ns square pulse. A random phase plate and steering wedges are used to produce a smooth, nearly flat-topped intensity distribution over a $700-\mu \mathrm{m}$-diam focal spot. The $x$-ray flux that emerges from the back of the Au foil impinges on a sample consisting of a $1000-\AA \mathrm{CH}$ layer on either side of a $200-\AA$ Fe sample. The angle, time, and frequency dependence of the $x$-ray flux from the back of the Au foil is known from previous measurements. ${ }^{15}$ Figure 2 shows the results of a radiation-hydrodynamic simulation used to calculate the sample temperature and density history in response to the x-ray flux. The curves represent the middle of the Fe sample; because of the $\mathrm{CH}$ tamping, the gradients in temperature and density are less than 5\% and 10\%, respectively. The results in Fig. 2 are corroborated by previous measurements ${ }^{16}$ of the ionization balance in the plasma as determined by absorption spectroscopy in the 100- to $200-\mathrm{eV}$ range. The $n=$ $3 \rightarrow 3$ features of Fe are predicted to be of major importance under these plasma conditions.

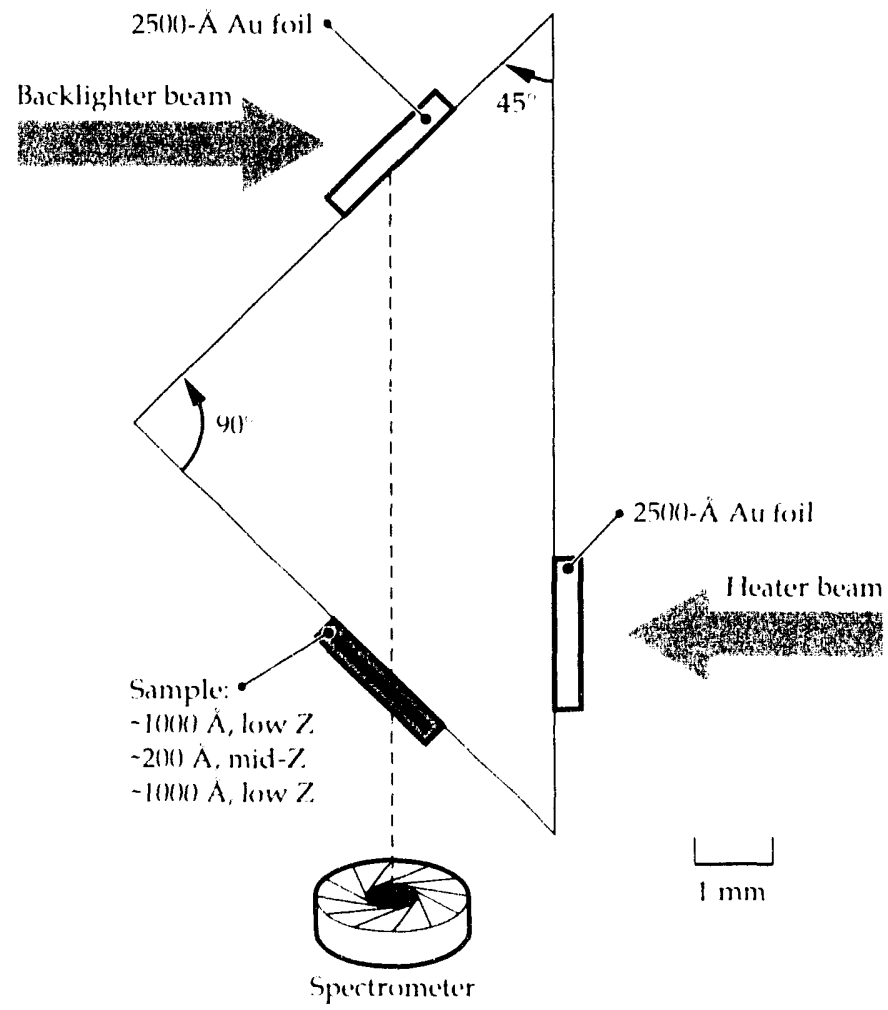

FIC.URI 1. Fxperimental arrangement. Key elements are the hedter and backlighter, their assexiated driving laner beams, the sample, and the time-resolved spectrometer.
A second $0.53-\mu \mathrm{m}$ laser beam, with a 2-ns square pulse, which can be arbitrarily delayed with respect to the first beam, is focused to a 300-mm-diam spot on another $2500-\AA$ Au foil, which is aligned on the axis formed by the spectrometer and the sample. This $x$-ray source, which is 10 times further away from the sample than the heater, is the absorption probe, or backlighter, whose transmission through the sample is time-resolved with the spectrometer. A variety of measurements were made to ensure the integrity of the Fe sample, ${ }^{15}$ with the following results: (1) Stray laser light impinging on the sample was less than $10^{8} \mathrm{~W} / \mathrm{cm}^{2}$, and therefore has negligible effect on it. (2) The sample is uniformly heated by the heater $x$ rays. (3) The expansion of the sample under $x$-ray heating was independently found to agree with hydrodynamic simulations. The $x$ rays heat the sample uniformly, so the radiation hydrodynamics are simpler than if the laser beam impinged directly on the sample.

The primary diagnostic instrument is a time-resolved spectrometer consisting of a Harada 1200 -line $/ \mathrm{mm}$ variable-line-space grating, 17 manufactured by Hitachi, coupled to an $x$-ray streak camera covering the spectral energy range from 50 to $150 \mathrm{eV}$. The grating has high efficiency at high order, ${ }^{18}$ so the unwanted high-order contributions were eliminated with an iridium-coated mirror at $11^{\circ}$ to $16^{\circ}$ grazing angle of incidence and a $1300-\AA \mathrm{Al}$ filter. The rejection is 140:1 in second order and 50):1 in third order at $78 \mathrm{eV}$, and 1000): 1 in second order and 45()0:1 in third order at $72 \mathrm{eV}$. The streak camera, which provides a continuous time history, has a fiber-optic timing fiducial, which permits the time histories of the absorption spectrum and the $x$-ray heating plasma to be related to within $30 \mathrm{pss}^{19}$
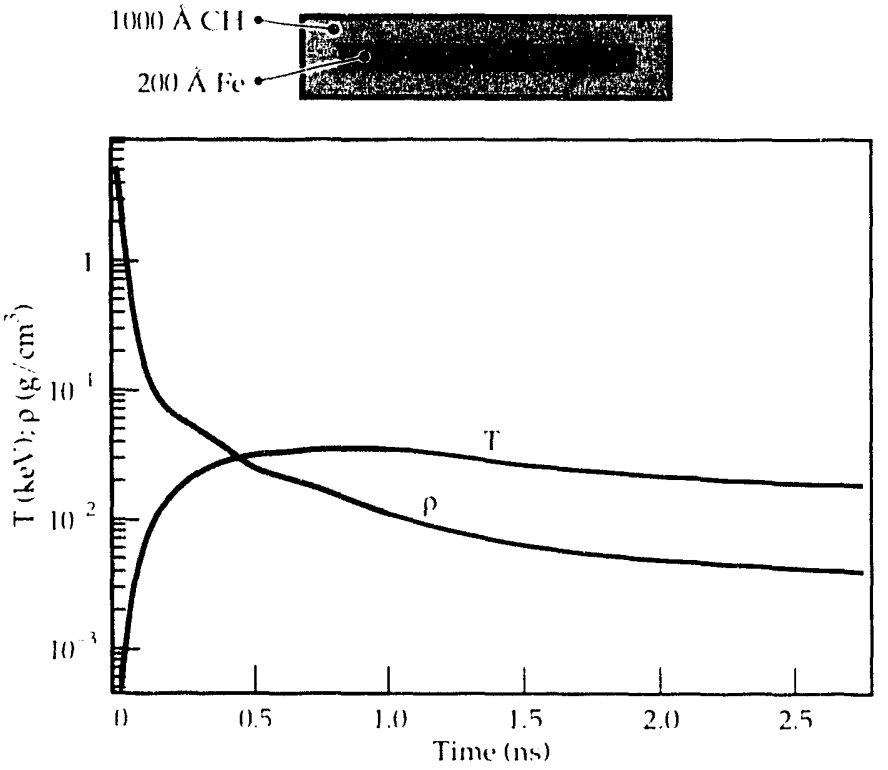

FICERI: 2. Simulation of temperature and density in the middle of the fo sample in responon to the measured $x$-ray flux from the heater. The shaded region indicates the duration of the heater pulse. 
Experiments were performed with various laser and sample configurations: (1) cold Fe tests using only a backlighter to measure its effects on the sample; (2) absorption of a pure $\mathrm{CH}$ sample using the heating $\mathrm{x}$-ray flux and the backlighter; (3) absorption of a Fe sample sandwiched between layers of $\mathrm{CH}$ using the heating $x$-ray flux and the backlighter; (4) emissivity of a $\mathrm{CH}$ sample using the heating $x$-ray flux only; and (5) $\mathrm{Fe}$ emissivity using the heating $x$-ray flux only. To ensure uniformity of the observed target, the spectrometer view in all these measurements was restricted to the central 500 - $\mu$ m-diam region of the foil.

The results can be summarized as follows. The coldFe test [No. (1), above] clearly showed a cold Fe opacity with some insignificant warming $2.0 \mathrm{~ns}$ after the start of the backlighter pulse. The $\mathrm{CH}$ and Fe opacity experiments [(2) and (3)] showed clear absorption spectra from heated matter, while the emissivity experiments [(4) and (5)] showed no absorption features. The data were analyzed by first converting film density to intensity using a calibrated step wedge. The emissivities of the $\mathrm{CH}$ and $\mathrm{Fe}$ samples were determined and subtracted from the $\mathrm{CH}$ and Fe sample absorptions, respectively. The resulting $\mathrm{Fe}$ spectrum was then divided, point by point, by the corresponding $\mathrm{CH}$ sample spectrum; this removes the $\mathrm{x}$-ray energy dependence of the detector and yields the transmission through the Fe only.

Figure 3 shows the unprocessed Fe emission and absorption spectra $1.9 \mathrm{~ns}$ after the beginning of the heater pulse. These spectra and the corresponding $\mathrm{CH}$ sample spectra were used to determined the Fe transmission shown in Fig. 4.

\section{Discussion and Conclusions}

Figure 4 shows the experimental results for iron and corresponding OPAL transmission calculations (using intermediate coupling for the configuration term structure). The figure also shows the results of a calculation that ignores the configuration term splitting and uses hydrogenic oscillator strengths, as in the Los Alamos

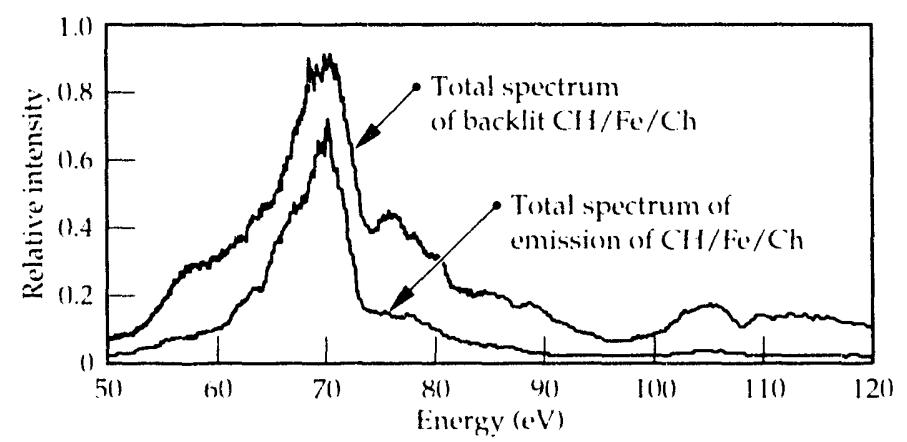

FICiURF 3. Measured x-ray spectrum for a tamped fe sample, with (upper) and without (lower) backlighter beam, both 1.9 ns after the heater pulse beegins. opacity codes. ${ }^{13}$ Only the OPAL calculation predicts the strong, broad spectral feature near $70 \mathrm{eV}$, which arises from $\Delta n=0$ transitions. Since the Rosseland mean opacity strongly weights regions of low photon absorption, omitting the $\Delta n=0$ transitions, or even including those transitions but neglecting term splitting, leads to a considerable underestimate of the iron opacity.

The temperature and density assumed in the OPAL calculations are only representative of the experimental conditions, but the code predicts the strong, broad $\Delta n=0$ features over a wider temperature and density range than the uncertainty in the experimental conditions. This type of direct experimental verification is important to the improvement of opacity models. ${ }^{20}$

\section{Notes and References}

1. A. Weiss, J. J. Keady, and N. H. Magee, "A Collection of Los Alamos Opacity Tables for All Temperatures," Atomic Data Nuicl. Ditn 45, 2(19-238 (199)).

2. F. J. Rogers and C. A. Iglesias, "Radiative Atomic Rosseland Mean Opacity Tables," Astrophys. J. Suppl. Sir. 79, 507 (1992)

3. A. N. Cox, S. M. Morgan, F. J. Rogers, and C. A. Iglesias, "An Opacity Mechanism for the Pulsation of OB Stars," Astrophlys. I. 393, 272-277 (1992); A. N. Cox, "Masses of RRd Variables Using Livermore OPAL Opacities," Astrophys. ). 381, L71-L.74 (1991); (i. Kovacs, J. R. Buchler, and A. Marom, "RR Lyrae Pulsations Revisited," Astront. Astrophlyss. 252, L27-L30) (1992); M. Kirlakidis, M. F. El Eid, and W. Glaztel, "Heavy Element Opacities and the Pulsations of $\beta$ Cephei Stars," Mon. Not. R. Astrom. Sox. 255, 1 (1992); P. Moskalik, J. R. Buchler, and A. Marom, "Toward a Resolution of the Bump and Beat Cepheid Mass Discrepancies," Astrophy/s. 1. 385, 685-69.3 (1992); P. Moskalik and W. A.

Driembowski, "New ()pacities and the Origin of the $\beta$ Cephei Pulsation," Astron. Astrophyss. 256, L5-L_8 (1992).

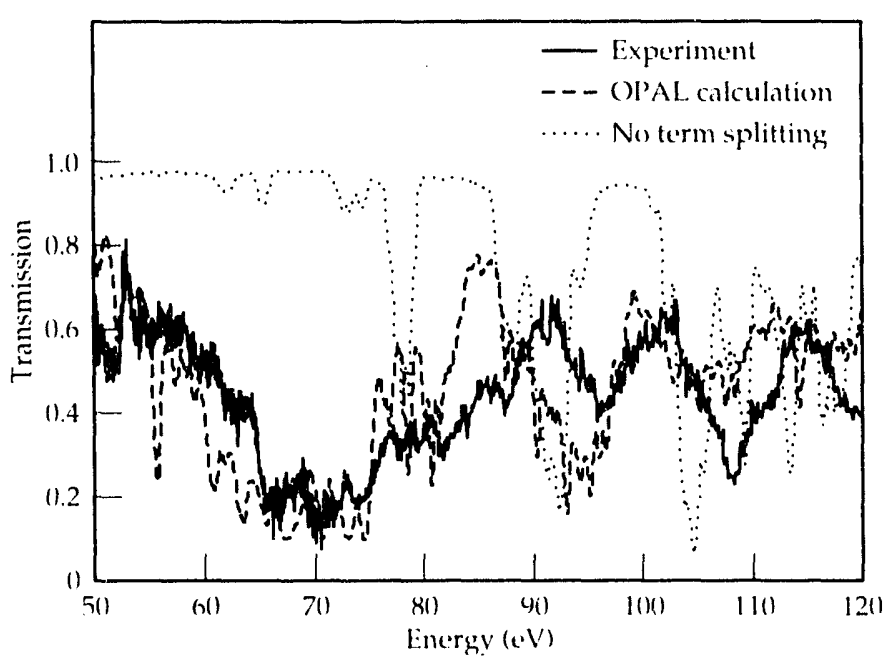

Ficiure 4. Measured transmission through the le sample at the same time as Fig. 3, and two transmission calculations at a temperature of $20 \mathrm{eV}$ and density of $0.1 \% 18 \mathrm{~g} / \mathrm{cm}^{3}$. The ()PAL calculation includes spin-orbit interactions in the configuration term structure calculations; that labeled "Noterm splitting" ignores lerm structure" and assumes hydrogenic oscillator strengths. 
4. D. B. Guenther P. Demarque, Y.-C. Kim, and M. H. Pinsonneault, "Standard Solar Model," Astrophys. J. 387, 372-393 (1992); W. A. Dziembowski, A. A. Pamyatnykh, and R. Sienkiewicz, "Seismological Tests of the Standard Solar Models Calculated with New Opacities," Astron. Astrophys. (in press).

5. R. B. Stothers, "Upper Limit to the Mass Of Pulsationally Stable Stars with Uniform Chemical Composition," Astrophys. J. 392, 706 (1992).

6. R. B. Stothers and C.-W. Chin, "Metal Opacities and Convective Core Overshooting in Population I Stars," Astrophys. J. 381, L67-L70 (1991); R. B. Stothers and C.-W. Chin, "Criterion for Convection in an Inhomogeneous Star," Astrophys. J. 390, 136 (1992).

7. F. J. Swenson, G. Stringfellow, and J. Faulkner, "Is There a Classical Hyades Lithium Problem," Astrophys. J. 348, L33-L36 (1990).

8. L. B. Da Silva, B. J. MacGowan, D. R. Kania, B. A. Hammel, C. A. Back, E. Hsieh, R. J. Doyas, C. A. Iglesias, F. J. Rogers, and R. W. Lee, "Absorption Measurements Demonstrating the Importance of $\Delta n=0$ Transitions in the Opacity of Iron," Phys. Re'r. Lett. 69, 438-441 (1992).

9. F. J. Rogers, B. G. Wilson, and C. A. Iglesias, "Parametric Potential Method for Generating Atomic Data," Phys. Ri'z. $\triangle 38$, 50)(07-5020 (1988).

10. R. D. Cowan, The Theory of Atomic Structure (University of California Iress, Berkeley, 1981).

11. F. J. Rogers, Proc. International School of Physics "Enrico Formi" Course CXIII, S. Eliezer and R. A. Ricci, Eds. (North Holland, Amsterdam, 1991); Astrophy/s. I. 310, 723 (1986).

12. C. A. Iglesias and F. J. Rogers, "Opacities for the Solar Radiative Interior," Astrophys. J. 371, 408-417 (1991).

13. C. A. Lglesias, F. J. Rogers, and B. G. Wilson, "Reexamination of the Metal Contribution to Astrophysical Opacity," Astrophys. I. 322, L45-L48 (1987); C. A. Iglesias, F. J. Rogers, and B. G. Wilson, "Opacities for Classical Cepheid Models," Astrophys. J. 360, 221-226 (199(1); C. A. Iglesias, F. J. Rogers, and B. G. Wilson, "Spin-Orbit Interaction Effects on the Rosseland Mean Opacity," Astrophys. I. 397, 717-728 (1992); C. A. Iglesias and F. J. Rogers, "Opacity Tables for Cepheid Variables," Astrophyss. I. 371, L.73-L75 (1991).
14. T. S. Perry, S. J. Davidson, F. J. D. Serduke, D. R. Bach, C. C. Smith, J. M. Foster, R. J. Doyas, R. A. Ward, C. A. Iglesicis, F. J. Rogers, J. Abdallah Jr., R. E. Stewart, J. D. Kilkenny, and R. i $\underset{v}{ }$. Lee, "Opacity Measurements in a Hot Dense Medium," Phys. Re'r. Lett. 67, 3784-3787 (1992); B. A. Hammel, D. R. Kania, R. J. Doyas, R. W. Lee, C. A. Iglesias, J. F. Seely, U. Feldman, and C. M. Brown, "Absorption Measurements of Radiatively Heated Low-Z Materials," Radiatize Propertic's of Hot De'lss' Matter, W. Goldstein, C. Hooper, J. Gauthier, J. Seely, and R. Lee, Eds. (Proceedings of the 4th International Workshop on Hot Dense Matter, Sarasota, FL, October 22, 1990); World Scientific, Singapore, 1991).

15. D. R. Kania, H. Kornblum, B. A. Hammel, J. F. Seely, C. M. Brown, U. Feldman, S. G. Glendinning, P. E. Young, E. J. Hsieh, M. A. Henesian, L. C. Da Silva, B. J. MacGowan, D. S. Montgomery, C. A. Back, R. J. Doyas, J. Edwards, and R. W. Lee, "Characterization of an X-Ray-Flux Source for the Production of High-Energy-Density Plasmas," Plyys. Rev' A 46, 7853 (1992)

16. B. A. Hammel, D. R. Kania, R. Doyas, R. W. Lee, and C. A. Iglesias," High Resolution XUV Absorption Spectra of Radiatively Heated CH Foils," Bull. Am. Physs. Soc. 36, 2416 (1991).

17. T. Kita, I. Harada, N. Nakana, and H. Kurada, "Mechanically Ruled Aberration-Corrected Concave Gratings for a Flat-Field Grazing-Incidence Spectrograph," Appl. Opt. 22, 512 (1983); G. P. Kiehn, O. Willi, A. R. Damerell, and M. H. Key, "Novel Time-Resolved VUV Spectrograph for X-Ray Laser Research," Appl. Opt. 26, 425 (1987).

18. J. Edelstein, M. C. Hettrick, S. Mrowka, P. Jelinsky, and C. Martin, "Extreme UV Measurements of a Varied Line-Space Hitachi Reflection Grating: Efficiency and Scattering," Appl. Opt. 23, 3267 (1984).

19. J. Koch and B. J. MacGowan, "Aluminum-Coated Optical Fibers as Efficient Infrared Timing Fiducial Photocathodes for Synchronizing X-Ray Streak Cameras," I. Appl. Phys. 69, 6935 (1991).

20. D. B. Guenther, "Astrophysics: Stellar Opacities in a Flash," Naturr' 359, 585 (1992). 


\title{
SOlid Hydrogen Surfaces
}

\author{
G. W. Collins \\ E. R. Mapoles \\ J. Hoffer* \\ J. Simpsont \\ J. Sanchez \\ R. Bell \\ W. Unites
}

\section{Introduction}

Future targets for ICF will require thick layers of hydrogen ice inside of a several millimeter diameter shell to reach the conditions necessary for ignition. For several years we have explored various opticns for making and characterizing the surface roughness of such layers. During an implosion, initial surface perturbations grow due to hydrodynamic instabilities and, if they are sufficiently large, will mix enough cold fuel with the center hot spot to prevent ignition. Since all National Ignition Facility (NIF) target designs will use deuterium-tritium (D-T) as the fuel, the ability to produce uniform D-T films inside ICF capsules is vital.

A promising approach to creating a solid layer, called beta layering, lets the intrinsic radioactive heat ing in D-T drive a solid layer towards a condition in which the inner surface is isothermal. If a spherical isotherm can be designed, the D-T ice will assume the configuration needed for ICF targets. We characterized the time dependence of this process previously ${ }^{1}$ and found it acceptable for cryogenic ICF targets.

In this paper, we address the question: Can a solid D-T layer be formed on the inside of a spherical shell with the uniformity required for an NIF? We cannot yet answer this question in the affirmative. Our series of D-T beta layer experiments show a surface roughness amplitude larger than that required for the proposed NIF. However, in nontritiated hydrogen layer experiments, we have observed significant, progressive $\mathrm{im}_{\mathrm{l}}$ )rovements in the surface finish. With continued progress, we will have the ability to form D-T layers to NIF specifications.

In the beta-layering experiments, the root mean square (RMS) surface roughness of a $100-\mu \mathrm{m}$-thick D-T film at roughly $18-19 \mathrm{~K}$ is about $2 \mu \mathrm{m}$ RMS over a 6 - $\mathrm{mm}$ length. From two different measurements, we find the RMS increases linearly with length. ${ }^{2}$ This is too rough by a factor of 4 for the proposed NIF target.

Because of the difficulty in forming D-T films, there is very little data on this subject. For this reason, we began a parallel series of experiments using a second cryostat, involving nontritiated hydrogen to map out the experimentally accessible phase space for producing uniform solid hydrogen films for baseline target designs of an NIF. We find near the triple point (the point where the solid, liquid, and gaseous states are in equilibrium) the surface roughness of $\mathrm{H}_{2}$ and $\mathrm{D}_{2}$ films decreases with increasing temperature and with increasing thermal gradient through the layer, normal to the surface. The underlying substrate affects the final density profile of thick hydrogen films primarily by setting the length scale for crystallite size. By scaling the density profile for a $100-\mu \mathrm{m}$-thick $D_{2}$ layer at $18.4 \mathrm{~K}$, the surface roughness is approximately $0.5-1.0 \mu \mathrm{m}$ RMS over a $6-\mathrm{mm}$ length.

There have been a few relevant experiments studying the properties of thin (monolayer to $10 \mu \mathrm{m}$ ) hydrogen films. Typically, the thin films are used as weakly interacting Van der Waals substrates ${ }^{3}$ for studying the mobility of surface state electrons or phase transitions of helium films. From electron mobility experiments, it has been estimated that for a $2-\mu \mathrm{m}$ layer the characteristic length scale for surface roughness is $200 \mathrm{~nm}$; the surface roughness amplitude can then be calculated to be $10 \mathrm{~nm}^{4-7}$ This is the only other hydrogen surface roughness estimate that exists. The bandwidth for this measurement is very short, but if we scale the result to a length of $6 \mathrm{~mm}$, assuming the RMS surface roughness scales linearly with length, we find an RMS of about $0.3 \mu \mathrm{m}$.

\footnotetext{
* Los Alamos National Laboratory
}

+ General Atomics 
Other experimenters have investigated KosterlitzThouless transitions of hydrogen, for example the roughening transition. ${ }^{8}$ Below the roughening temperature, crystals tend to have well-defined facets with sharp edges. Above the roughening temperature, the crystals are rounded and a thin mobile film wets the solid-vapor interface. Estimates from extrapolating critical points for successive monolayers of $\mathrm{H}_{2}$ place this temperature at about $74 \%$ of the triple point. ${ }^{9}$ Knowing this temperature for D-T could be useful for ICF targets since, above the roughening temperature, the entropy term in the surface free energy may overcome the energy stabilizing sharp facets, and allow the hydrogen to conform uniformly to the shell curvature.

\section{Experimental Apparatus}

D-T ice surfaces have been photographed and characterized here and at Los Alamos National Laboratory (LANL). The Livermore experimental system used to refrigerate the target and load D-T has been described previously. ${ }^{1}$ In these experiments, the sample container was a $6 \mathrm{~mm}$ [outer diameter (OD)] by $5 \mathrm{~mm}$ [inner diameter (ID)] sphere made by gluing two hemispheres of single crystal quartz together. The single crystal quartz was used for high thermal conductivity. The sphere was supported with a $0.02 \mathrm{in}$. OD stainless steel tube glued into a slot in one of the hemishells; it also served as a fill tube for loading D-T. Figure 1 shows an empty assembly before the D-T is loaded.

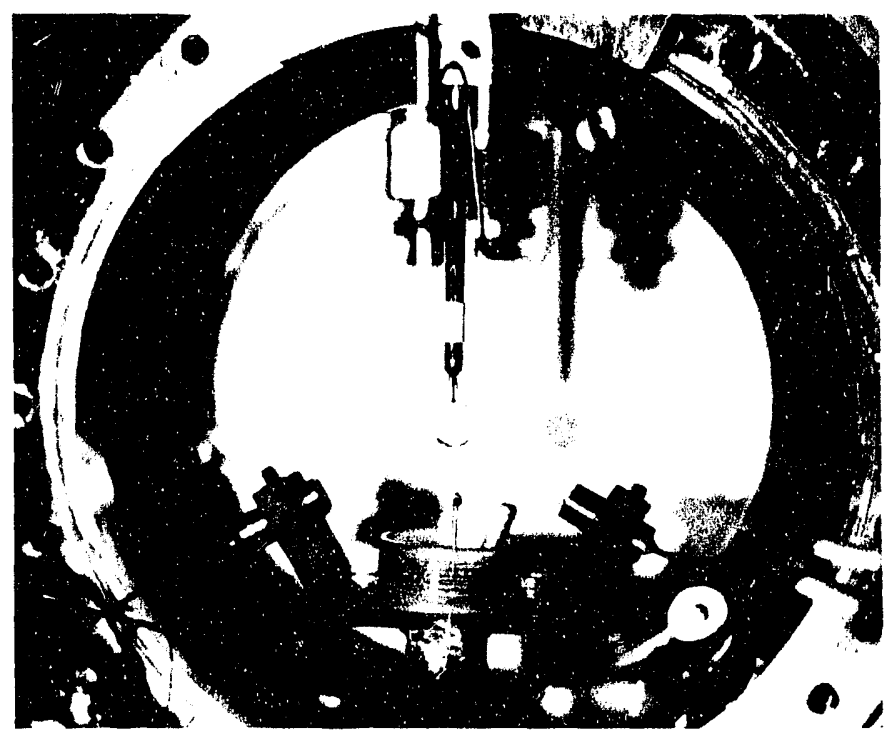

FICURE 1. Photegraph of the experimental assembly. The 6-mm quartz shell in the center hangs from the D-T fill line. A large light diffuser is in the background.
The LANL experiment used a cylinder rather than a sphere to eliminate distortion in the image caused by refraction at the edge of a spherical container. Figure 2 shows the container. A central 2-mm-diam cylindrical section is bored in a block of copper. A counterbore $4 \mathrm{~mm}$ diam and $1 \mathrm{~mm}$ deep is added at both ends and is sealed with sapphire windows. The larger reliefs force the D-T ice to curve away from the D-T surface in the inner section. This part of the D-T surface can be imaged without looking through the curved ice. The D-T must still be observed through D-T ice on the inside of the sapphire window, but this ice is flat and normal to the viewing axis. The cell is supported in a can that is filled with helium exchange gas to cool the sample chamber. The can is attached to a closed cycle refrigerator which allows the system temperature to be lowered to $12 \mathrm{~K}$.

Images at both LANL and LLNL are taken with a camera that uses a CCD array. The array has $1340 \times 1037$ pixels, which are each $6.8 \mu \mathrm{m}$ square. All of the images analyzed were $1024 \times 1024$ pixels stored with 16 bits per pixel. The images are downloaded to a personal computer for storage and later analysis. Figure 3 shows the LANL cylinder containing about $75 \mu \mathrm{m}$ of D-T, while Fig. 4 shows an image of the quartz shell containing about $100 \mu \mathrm{m}$ of $\mathrm{D}-\mathrm{T}$ ice inside.

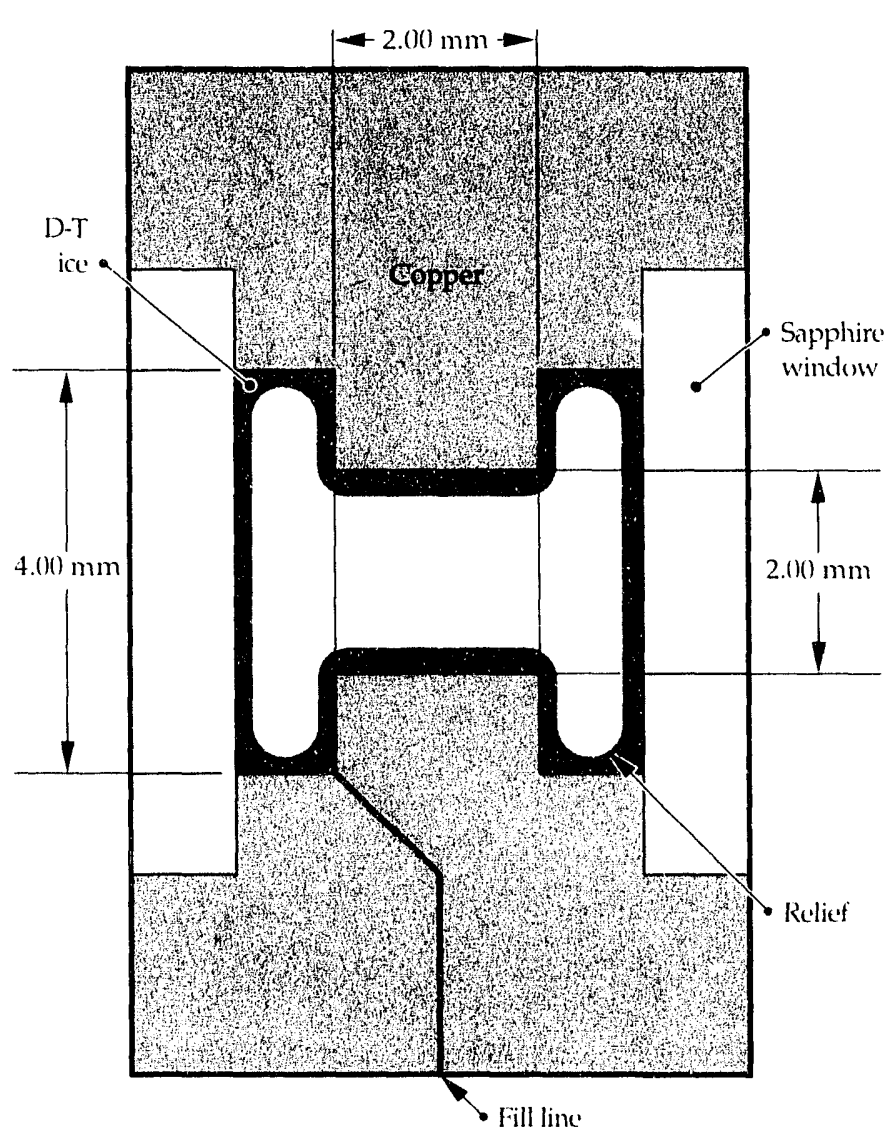

FIC,URE: 2. The LANL cylindrical D-T container. 
The D-T ice in the quartz ball had two days of ${ }^{3} \mathrm{He}$ build up before this particular experiment. From this and previous experiments, we know that the layering time for D-T of this age is about $35 \mathrm{~min}$. The ice was allowed to layer for about $5 \mathrm{~h}$ before final images were shot and analyzed. The layering in the LANL experiment was complicated by the ice on the sapphire window; a wait of about $12 \mathrm{~h}$ occurred before the ice became clear enough to obtain a useful image. The ice on the front window added some noise to the image. Therefore the roughness derived below must be regarded as an upper bound to the roughness. Future experiments will eliminate this problem.

A second LLNL cryostat, used to study non-tritiated hydrogen, was based on a open cycle helium flow refrigerator. The cold tip of this refrigerator was thermally connected to the sample cell (Fig. 5). This system is capable of working at temperatures between 4.4 and $40 \mathrm{~K}$ and is instrumented with thermometers on the copper sample cell and a window. The thermal stability was better than $0.01 \mathrm{~K}$ over a $10-\mathrm{min}$ period. The sample cell and radiation shield were machined out of copper. All the windows were made of single crystal sapphire cut along the nonbirefringent axis. The inside surface of one sample cell window (Fig. 5) was uncoated while the others were each coated with a different material: $15 \mathrm{~nm}$ of sputter coated $\mathrm{Be}, 750 \mathrm{~nm}$ of plasma polymer plastic $\mathrm{CH}_{1.3}$, or $100 \mathrm{~nm}$ of $\mathrm{MgF}_{2}$, so that we could study the substrate effects on the hydrogen ice morphology. All other window surfaces were coated with standard antireflective coatings. The hydrogen gas fill lines (Fig. 5) were made of copper capillary tubing, which were

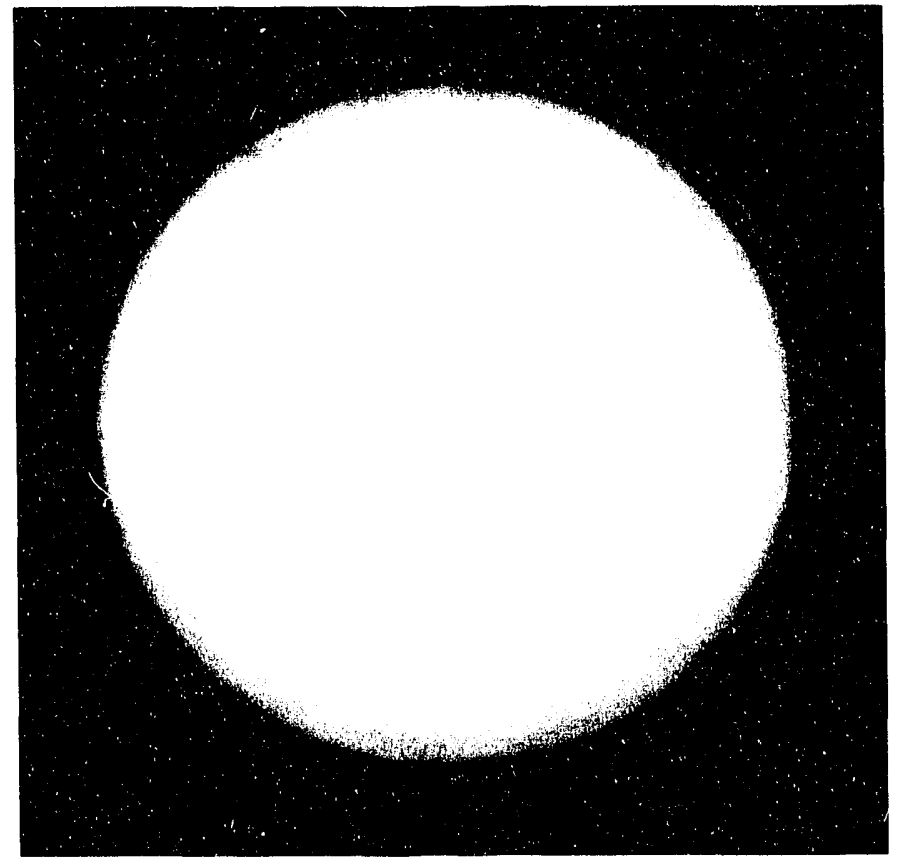

FICURE 3. D-T ice inside the LANL cylindrical container. wrapped in aluminized mylar and thermally isolated from the sample cell. Heaters were attached to the fill lines so that we could deposit hydrogen from the gas phase while the sample cell temperature was well below the freezing point. The two heaters shown in Fig. 5 were

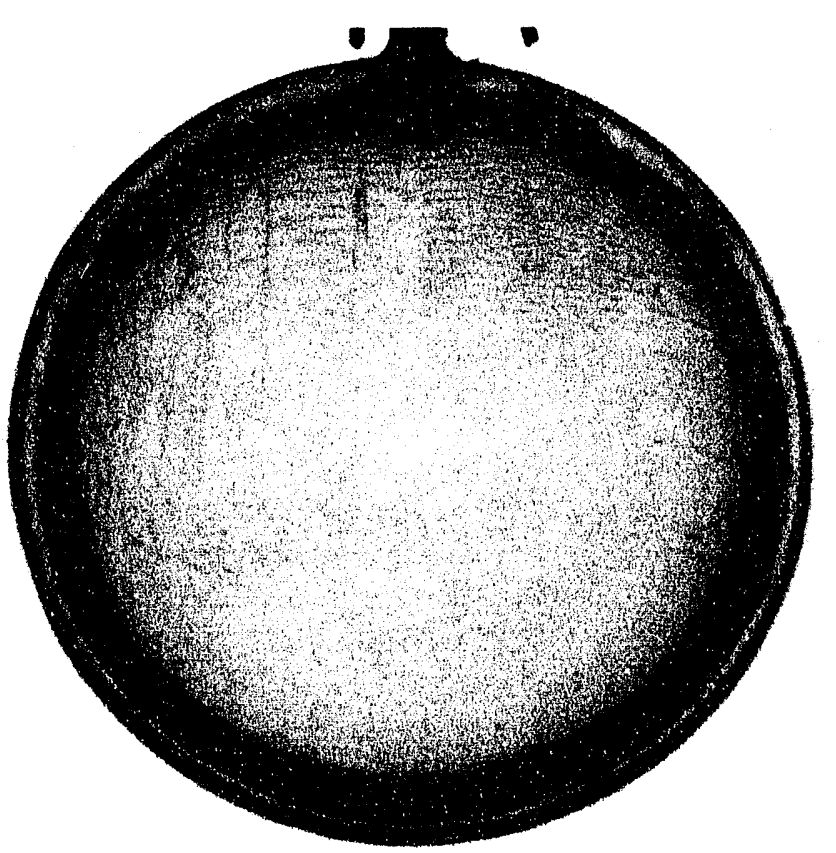

FICURE 4. The quartz shell containing D-T ice.

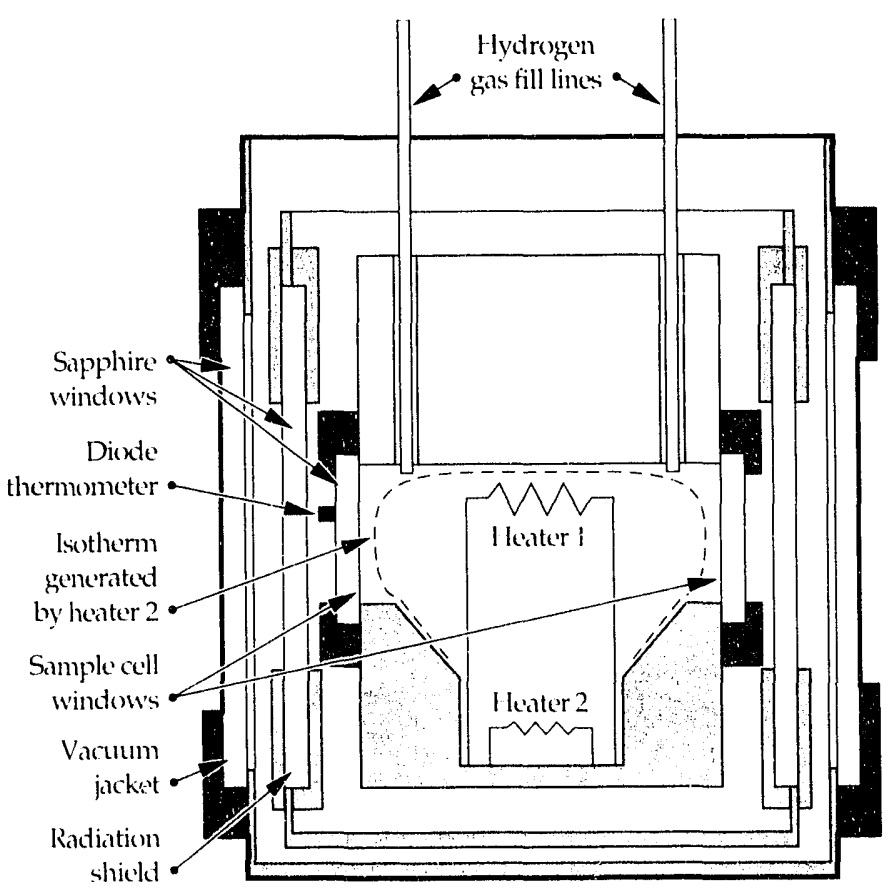

FICURE 5. Midplane sketch of the oryestat used in the interferometric study of solid hydrogen hyers. The cryostat has four fold symmetry: 
flat resistors wrapped from resistive wire. They were used to shape the isotherms and thus the solid hydrogen layers inside the sample chamber.

Two deposition techniques were used to grow the solid hydrogen films. In the first deposition technique (method 1), a constant volume of hydrogen gas is cooled until it reaches the liquid-gas phase boundary and then, as the gas cools further, it follows the saturated liquidvapor curve through the triple point into the solid phase. If a small thermal gradient is applied (from top to bottom in Fig. 5) while cooling near the triple point, the hydrogen in the saturated liquid can be forced to crystallize on the substrates. When the sample is further cooled below the triple point, these crystals undergo a large volumetric contraction, resulting in several strained and cracked crystallites. Depending on the temperature and thermal gradient through the layer, the crystal defects can be minimized by annealing. In the second deposition technique (method 2), hydrogen is solidified on the bottom of the sample cell, while the window substrates are free of hydrogen crystals. Then hydrogen crystals are grown onto the substrates through the vapor phase by creating a set of isotherms that differ from: the initial hydrogen ice profile. At temperatures below the triple point, the general shape of the solid hydrogen layer is believed to be determined by the sample cell isotherms. Physically, one can think of the thermal gradient through the hydrogen layer as a set of isothermal surfaces. Surface nonuniformities above $t_{1} x$ mean surface height lie on a higher temperature isotherm relative to the mean, resulting in a higher vapor pressure relative to the mean. This drives a redistribution of the solid surface to conform more closely with the mean isotherm. It is the roughness or deviation of the solid hydrogen from the smooth isotherms of the empty container that we have characterized in this paper.

The isotherms in this flow cryostat cell are controlled by heaters \#1 and \#2 (Fig. 5) and the liquid He flow rate. We calculated the characteristic isotherms and the heat flux through the hydrogen ice as a function of the power applied to heater $\# 2$. The isotherm calculations were performed assuming a fixed amount of hydrogen gas $(\sim 10)$ torr) in the sample cell and no solid hydrogen. In Fig. 5, an isotherm inside the sample cell shows the general shape of the hydrogen ice contour.

For quantitative analysis of the hydrogen ice profile, we use a digital phase measuring interferometric technique similar to that used by Bruning et al. ${ }^{11}$ The interferometer is a simple variation of the Mach-Zender design that includes a movable mirror in one arm, allowing the reference beam to be phase-shifted over several cycles with an accuracy of better than $\lambda / 20$. Each interferogram is collected with a microscope focused onto the solid hydrogen surface, producing an image on a CCD array. The magnification of the microscope varied between 2 and 3.5 with numerical apertures of 0.16 and 0.19 and working distances of $300 \mathrm{~mm}$ and $160 \mathrm{~mm}$, respectively. The depth of field was typically $800 \mu \mathrm{m}$. The CCD camera has $754 \times 488$ picture elements, each of which is about $12 \mu \mathrm{m} \times 14 \mu \mathrm{m}$. Both the automatic gain control and the gamma correction were turned off. The camera signal is converted to an analog signal and then redigitized with about 8 bits of dynamic range. Finally, after summing the three color planes, the image is stored on a computer.

To produce an optical path density (OPD) map of the solid hydrogen films, we collected several interferograms, each having a different reference phase. The reference phases were determined by stepping a piezodriven mirror in the reference beam path a fraction of a wavelength. The intensity of each interferogram can be written in two representations:

$$
\begin{aligned}
I(x, y, l) & =a^{2}+b^{2}+2 a b \cos k[u(x, x, y)-l] \\
& =a_{0}(x, y)+a_{1}(x, y) \cos (k l)+b_{1}(x, y) \sin (k l),
\end{aligned}
$$

where $x$ and $y$ are the planar dimensions of the wave front, $z(x, y)$ represents the OPD profile of the solid hydrogen film, $l$ is the mean path length difference between the reference and sample beams produced by the modulation of the piezo mirror, and $k$ is $2 \pi / l$. The first representation of the intensity distribution comes from the square of the sum of the reference wave front amplitude $u w_{1}=n\{\exp [-i k z u(x, y)]\}$ and the sample wave front amplitude $\left.w_{2}=b / \exp [-i k w(x, y)]\right\}$. The second representation is the Fourier series with the dc term and first harmonics only. By Fourier transforming the set of interferograms along the reference phase axis, we obtain the OPD through the hydrogen film within $2 \pi$ radians

$\pi(x, y)=\tan ^{-1}\left(b_{1} / a_{1}\right) / k$.

This OPD through solid hydrogen is calculated for an index of refraction of one. To transform it into a height profile for the hydrogen layer, we must scale $z(x, y)$ by the relative index of refraction of solid hydrogen (0.1). When the relative index of hydrogen is taken into account, an equivalent $2 \pi$ variation in phase corresponds to a $6.3-\mu \mathrm{m}$ variation in the hydrogen surface. When there is more than a $2 \pi$ variation in the roughness, tilt, or curvature of the hydrogen film, the real surface must be reconstructed to extend the dynamic range of the OPD measurement. This reconstruction is performed by detecting the nearest neighbor pixel jumps of about $3 \mu \mathrm{m}$ or more and adding or subtracting $6.3 \mu \mathrm{m}$ to these phase wrapped pixels. The resolution of this measurement was about $0.1 \mu \mathrm{m}$ RMS and was set by the long term stability of the interferometer. The background varied between 0.1 and $0.5 \mu \mathrm{m}$ RMS, 'epending on the substrate used. 
In the data presented we have not subtracted out this background. A standard technique for removing the background contribution is to subtract the amplitude and phase information of the "surface" profile. Because these experiments sometimes lasted over the period of days and the microscope was refocused as the hydrogen ice grew, we could not retain this background phase information. The background RMS roughness was $0.25 \mu \mathrm{m}$ for the $D_{2}$ data, $0.45 \mu \mathrm{m}$ for the $\mathrm{H}_{2}$ data on Be, and $0.15 \mu \mathrm{m}$ for the rest of the $\mathrm{H}_{2}$ data. Since part of this background is added to the surface roughness shown in the analysis below, the results should be considered upper bounds on the actual roughness.

\section{Analysis of D-T Photograph Images}

Once the images have been shot and stored, the objective of the experiment is to extract an estimate of the surface roughness from the stored data. We developed software that locates the surface of the D-T and the edge of the container in the image as a function of angle. The resultant 1-D estimate of the ice thickness can be Fourier transformed to estimate the power spectrum of perturbations on the ice.

The software begins by taking an estimate of the center of the container in the image and drawing a ray from the center to the edge. At one pixel increments along the ray, the intensity is estimated by fitting a plane to the four neighboring pixel values and interpolating to fix the intensity at the point on the ray. This is done for a series of rays offset by a given angular increment to generate an image of the target in polar coordinates (Fig. 6). Each column in this image is then processed to locate a feature associated with the inner edge of the D-T ice and the edge of the container. The difference between the two points represents the thickness of the D-T ice at a particular angle. The estimates of the locations of the edge of the container are averaged to give a new value of the center of the container, and the image is reprocessed until the estimates converge.

Figure 7 is a plot of the intensity of the pixels at a given angle for the image shown in Fig. 3. There are two large steps in the intensity, one at the outer edge of the D-T and one at the edge of the container. Differentiation converts the inflection points of these steps to minima which are located by using an algorithm to find the minima in each ray. The minima are sorted by depth for each ray to give an array of locations as a function of angle for each feature. Figure 8 shows the layer thickness as a function of angle for an LANL image. Each value of the layer thickness plotted is an average of six estimates.

A very similar but slightly more complex process was used for LLNL images such as that shown in Fig. 4. The outside of the quartz container was located using the technique described above. Because refraction at the inner edge of the hydrogen ice creates an intensity minima in the spherical geometry, no differentiation is done before locating minima when searching for the ice edge. The lower contrast in these images resulted in some discontinuities in the inner contour; the minima were resorted in order to maximize the continuity of the estimates of the D-T ice surface. Figure 9 is the resultant ice

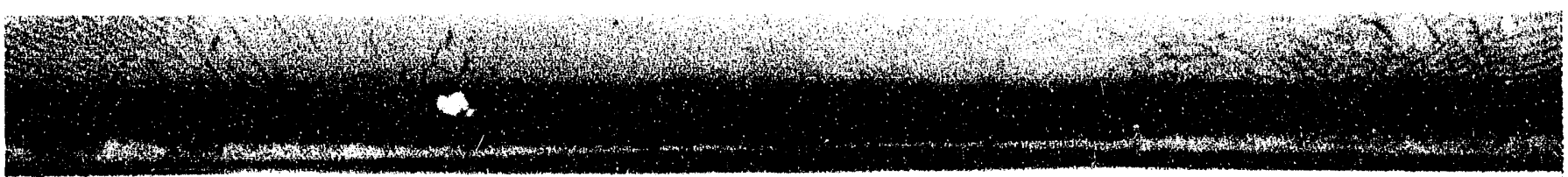

FICiURE 6. Photegraph of Fig. 4 after the edge of the container has been converted to polar courdinates with the center of the capsule as the center of the new courdinate system. The outside of the shell is marked with white pixels and the inside of the ice is morked with black pixels.

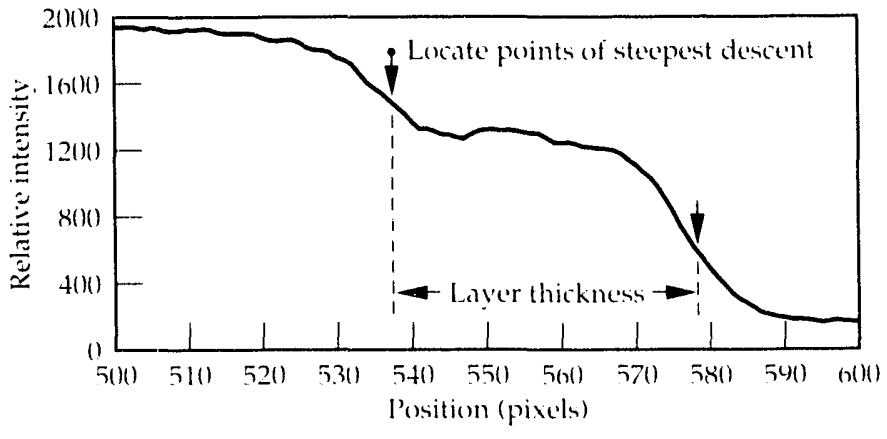

FICiUR: 7. An individual lineout from Fig. 3.

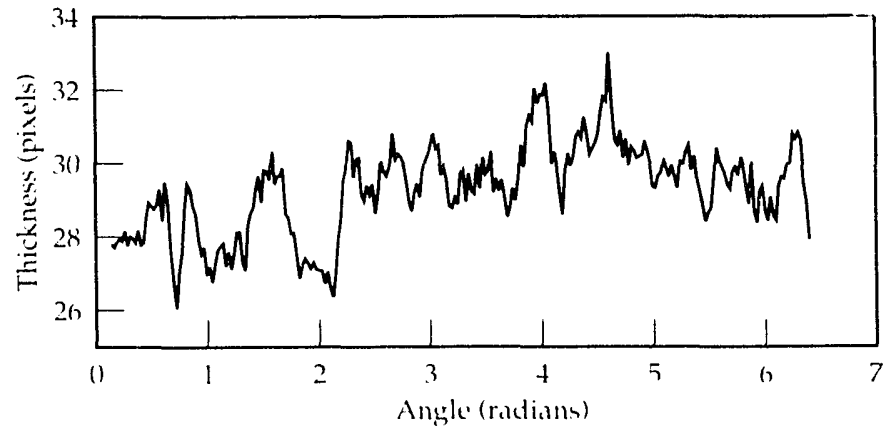

FIG:URE: 8. The ice surface from Fig. 3. 
thickness profile from the analysis of Fig. 4. Here ten estimates are averaged to give each point in the plot. About $20^{\circ}$ along the circumference of the target was excluded from the analysis because the image is distorted by the fill tube in this region.

These profiles can be Fourier transformed and the results used to calculate power spectra for the surfaces. These are shown in Fig. 10 for the two images of Figs. 3 and 4 . The spectra have the general form of $1 / f \gamma$, where $f$ is the frequency of the surface perturbation and $\gamma$ is in the range of 1-2. The roughness of the LANL surface for the first 128 modes is $2.1 \mu \mathrm{m}$ RMS. For the LLNL quartz image, the roughness is $5.7 \mu \mathrm{m}$ RMS after removing $\mathrm{P} 1$. The two roughnesses differ by a factor of 2.7 whereas the length scales differ bv 2.5. This is consistent with a linear scaling of the KMS surface roughness with length.

\section{Interferometric Results of $\mathrm{H}_{2}$ and $\mathrm{D}_{2}$}

The $\mathrm{H}_{2}$ and $\mathrm{D}_{2}$ surfaces were grown by the two methods described above. For both methods, hydrogen ice initially grows to form a rough layer and then anneals

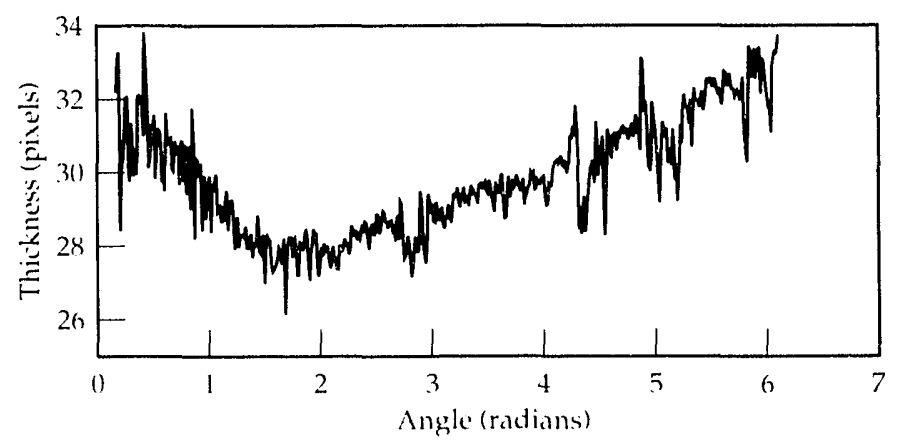

FICiURF 9. The ice surface from Fig. 4.

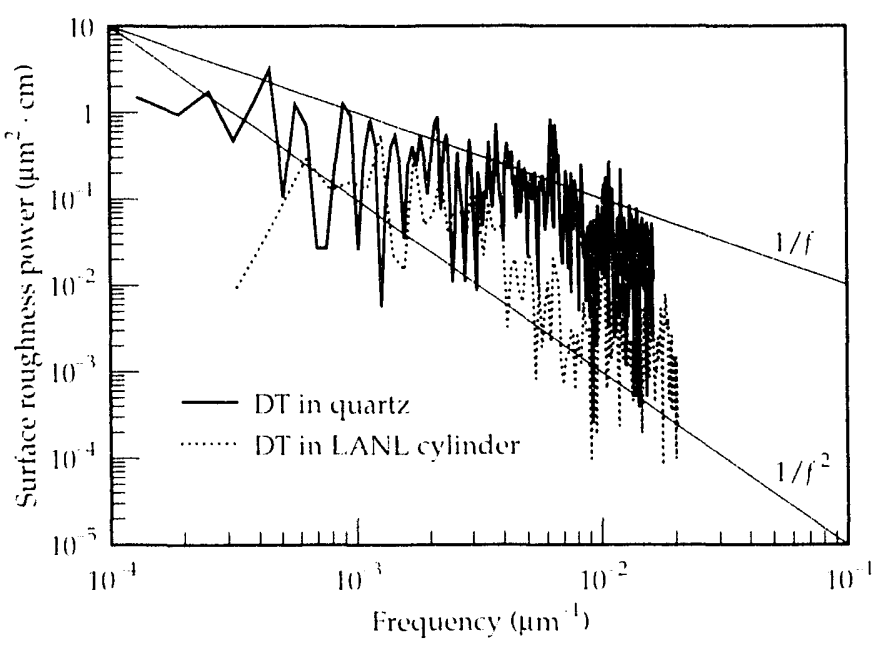

FIC CURE: 10. The power spectra of the surfaces of Figs. 8 and $y$. into a relatively smooth film, which follows the curved isotherm surface described above. Figure 11 shows the time evolution of the surface roughness for $175-\mu \mathrm{m}$ thick $\mathrm{H}_{2}$ films grown onto beryllium with method 2 at $12.0 \mathrm{~K}$ and $13.0 \mathrm{~K}$, and with a calculated heat flux of $421 \mu \mathrm{W} / \mathrm{cm}^{2}$ passing through the layer. The measurement covers the length scales between 410 and $6.4 \mu \mathrm{m}$. This plot shows the general time dependence of the density profile and the increase at late times that is characteristic for many of the surfaces grown in our experiments. From the power spectra at different times during this surface evolution, we find that near the maximum of the roughness, the spectral components at wavelengths shorter than $1 / 30 \mu \mathrm{m}$ fall off rapidly with frequency, roughly as $1 / f^{3}$, while near the minimum of the roughness evolution, these spectral components fall off much more slowly, close to $1 / f$. To compare surfaces grown under different conditions (temperature, heat flux, underlying substrate) we compare measurements at roughly equal time near the minimum of the roughness vs time evolution.

Figure 12(a) shows the roughness as a function of temperature normalized to the triple point for $D_{2}$ and $\mathrm{H}_{2}$ grown under different experimental conditions. The heat flux passing through the $\mathrm{D}_{2}$ layer was 18 $\mathrm{mW} / \mathrm{cm}^{2}$, the thickness was $100 \mu \mathrm{m}$, the substrate was $\mathrm{MgF}_{2}$, and the lateral detection bandwidth was $6.4 \mu \mathrm{m}$ to $410 \mu \mathrm{m}$. This film was grown by method 2 at $18.4 \mathrm{~K}$, and then lowered to the other temperatures shown in Fig. 12(a). The sample sat at constant temperature till the surface roughness reached a steady state value. ${ }^{11}$ The $\mathrm{H}_{2}$ layers grown onto beryllium using deposition method 2 were about $175-\mu$ m-thick, the heat flux was $421 \mu \mathrm{W} / \mathrm{cm}^{2}$, and the lateral detection bandwidth was 6.4 to $410 \mu \mathrm{m}$. Finally, the $\mathrm{H}_{2}$ layer grown onto $\mathrm{MgF}_{2}$ using method 1 (cooling through the triple point at the rate $\sim 0.1 \mathrm{~K} / \mathrm{s}$ to $12 \mathrm{~K}$ ) was about $10(0)-\mu \mathrm{m}$-thick, and the

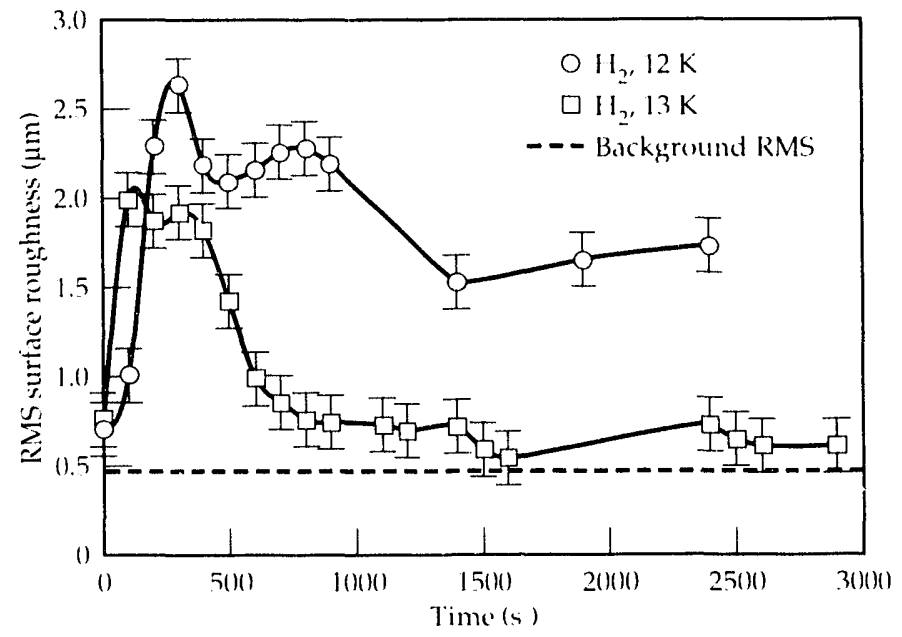

FIciURI: 11. RMS surface roughness i's time for $1 l_{2}$ films grown through the gals phase. 
heat flux passing through this layer was $42 \mathrm{~mW} / \mathrm{cm}^{2}$. Typically, solidifying $\mathrm{H}_{2}$ this rapidly results in a rough layer but, because of the large heat flux, the sample annealed to form a relatively smooth layer. The lateral detection bandwidth for this data was 14 to $896 \mu \mathrm{m}$. The general result from Fig. 12(a) is that the roughness is a decreasing function of temperature regardless of the technique or substrate used to grow the layer. This data supports the idea that increasing the entropy term in the free energy allows the hydrogen to conform more closely to a curved isotherm. When the background amplitude is subtracted from this data, the values are significantly lowered, and we can fit the RMS to the function RMS $=a^{*} \exp (b / T)$, where $a$ and $b$ are fit parameters. We find $b=319 \mathrm{~K}$ for the $\mathrm{H}_{2}$ data and $434 \mathrm{~K}$ for the $D_{2}$ data. This is interesting because the ratio of
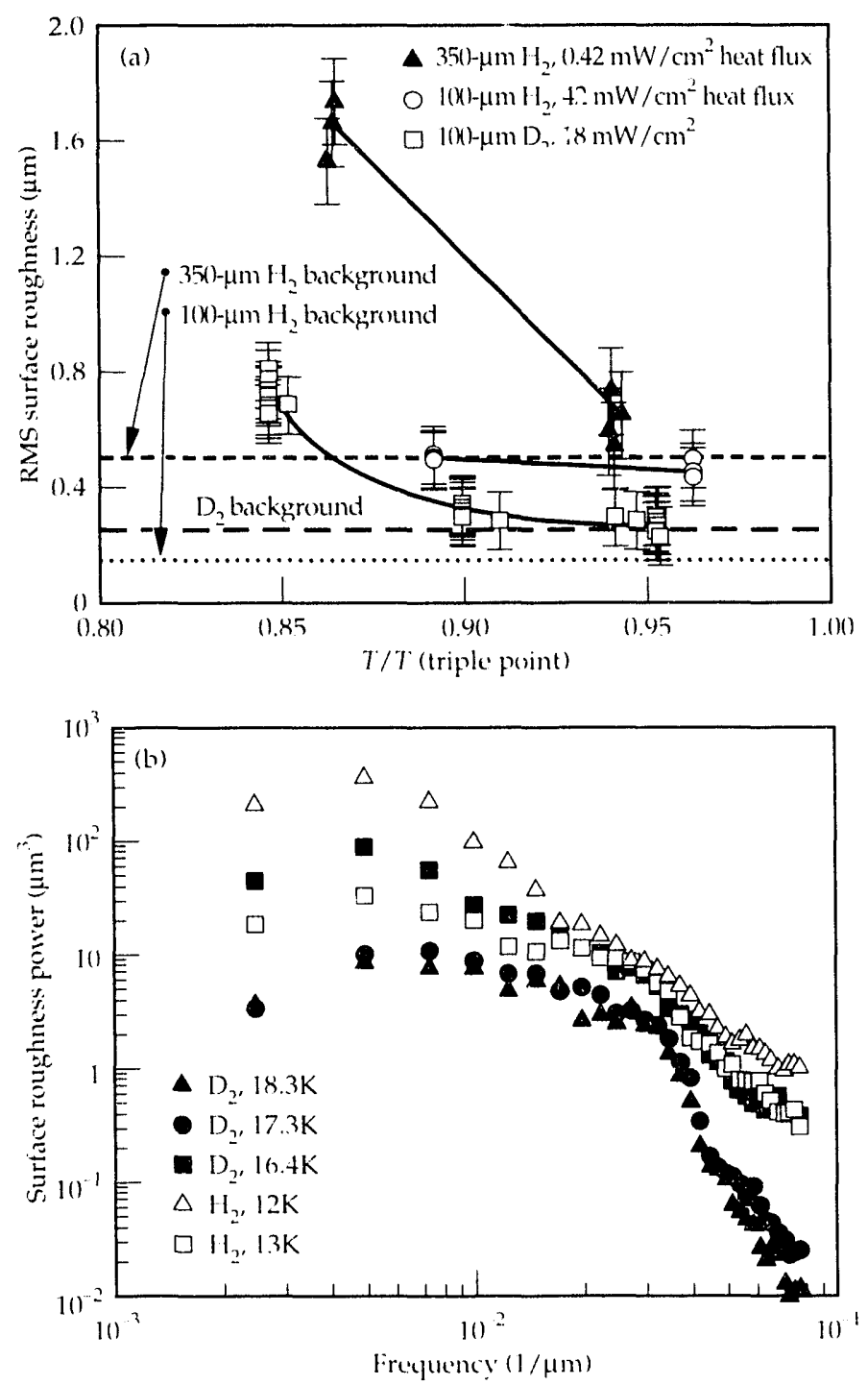

FICiURE 12. (a) RMS surface roughness in temperature. (b) Power spectrum of the surface roughness is temperature. these numbers scales roughly as the square root of the mass, and is similar to the bulk diffusion activation energies for hydrogen. The activation energy as measured by nuclear magnetic resonance for the bulk molecules is $190 \mathrm{~K}$ for $\mathrm{H}_{2}$ and $276 \mathrm{~K}$ for $\mathrm{D}_{2}$. With this simple comparison, we speculate that the surface roughness is related inversely to the diffusion of the molecules and that the activation energy for the molecules at the surface is about $60 \%$ larger than the molecules in the bulk.

In Fig. 12(b), we plot the spectral components of the surface roughness for the $\mathrm{H}_{2}$ and $\mathrm{D}_{2}$ films grown from the gas phase as described above. As the temperature increases, the spectrum tends to flatten. For $\mathrm{D}_{2}$ at $18.4 \mathrm{~K}$, the power at frequencies below $1 /(30 \mu \mathrm{m})$ falls off roughly as $1 / f$ while at $16.4 \mathrm{~K}$ the power falls off closer to $1 / f^{2}$. Also in the $D_{2}$ runs, we observed the emergence of a correlation length defined by the change in the scaling behavior at about $1 / 30 \mu \mathrm{m}$.

Figure 13 shows the roughness vs the calculated heat flux through the layer. The $\mathrm{H}_{2}$ layer was grown by method 1 onto $\mathrm{MgF}_{2}$ and cooled at the rate of $\sim 0.1 \mathrm{~K} / \mathrm{s}$ to $12 \mathrm{~K}$; the lateral bandwidth was $14 \mu \mathrm{m}$ to $896 \mu \mathrm{m}$ and the film thickness was about $100 \mu \mathrm{m}$. The $\mathrm{D}_{2}$ layer was grown by method 2 on $\mathrm{MgF}_{2}$ at $18.4 \mathrm{~K}$; the lateral detection bandwidth was 6.4 to $410 \mu \mathrm{m}$ and the film thickness was about $100 \mu \mathrm{m}$. These data show a steeply decreasing roughness with increasing heat flux, similar to the roughness vs temperature behavior shown in Fig. 12(a). The thermal gradients generated by an external heater can be compared to the thermal gradients in D-T created by the tritium radiation. For a $100-\mu \mathrm{m}$-thick D-T layer,

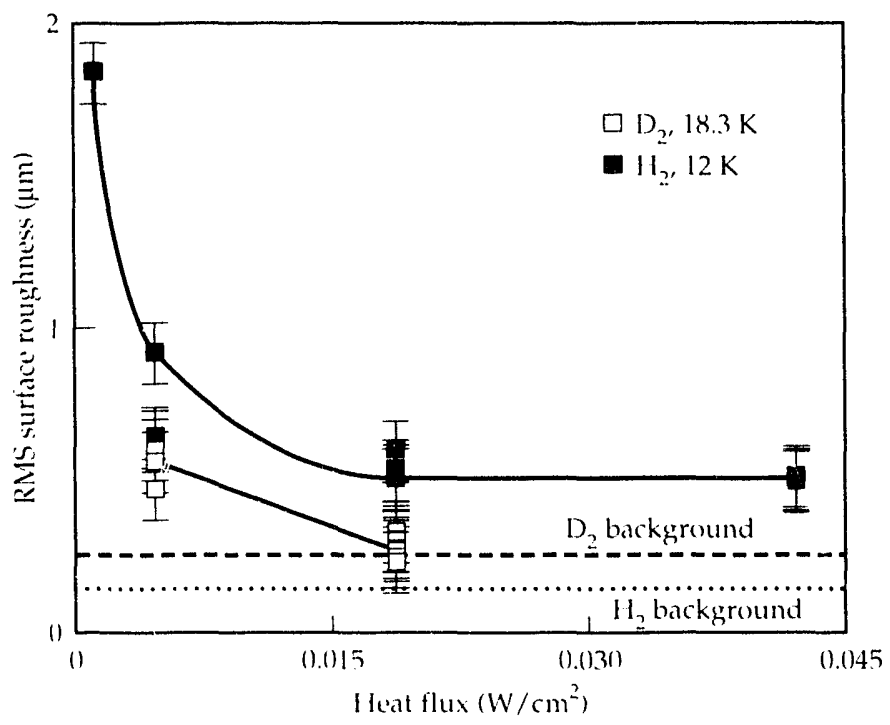

FICiLRE 13. RMS surtace roughness is heat flux through the laver (normul to the surface) 
the thermal gradient is about $0.0008 \mathrm{~K}$. For a $100-\mu \mathrm{m}-$ thick $D_{2}$ layer with a heat flux of $0.001 \mathrm{~W} / \mathrm{cm}^{2}$, the thermal gradient is $0.003 \mathrm{~K}$. Thus for most of our samples, the thermal gradient is at least a few times that of D-T.

There are many other factors that effect the OPD of a hydrogen layer, including the underlying substrate and the rate of crystal growth. We studied the effects of three substrates: $\mathrm{CH}_{1.3}$, Be, and $\mathrm{MgF}_{2}$ on the nucleation and the structure of thick layers. Generally, the substrate affects the scale of the underlying crystal size while the surface roughness of smooth patches is similar for the different substrates. At about $17 \mathrm{~K}$, the $\mathrm{D}_{2}$ crystals grow with sharp facets on Be until the entire substrate is covered, while, on plastic, $D_{2}$ grows from nonfaceted mounds to just below $16.5 \mathrm{~K}$. This gives rise to many more crystals and crystal defects on $\mathrm{CH}$ than Be. For most samples, we can remove the majority of crystal defects by increasing the temperature and thermal gradient through the film and we are working on ways to implement these techniques in ICF capsules.

\section{Mix Analysis}

Having calculated a power spectra for a D-T ice surface, we would now like to know how this roughness impacts the performance of an ICF capsule. For this analysis we use the LANL data of Fig. 9, since this data was taken using a container of about the right size. This data was modeled using a 1-D power spectrum of the form

$$
P_{1-\mathrm{D}}(l)=\frac{(55.0 \mu \mathrm{m})^{2}}{(10+l)^{2}}
$$

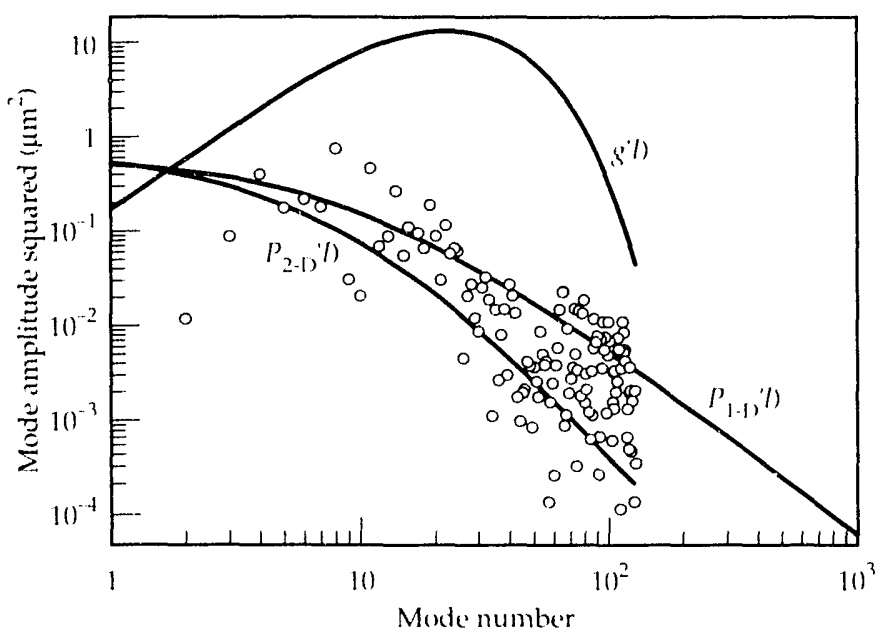

FlciURE: 14. 1- and 2-1) power spectra and growth factors for the ice of Fig. 8 . where the normalization factor was chosen to match the total power. The fit and data are shown in Fig. 14. In order to compute the effects of the implosion on these perturbations the 1-D spectrum must be converted to an equivalent $2-D$ power spectrum ${ }^{12}$ using

$$
P_{2-\mathrm{D}}(l)=-\frac{d}{d l} \int_{0}^{\infty} P_{1-\mathrm{D}}\left(\sqrt{n^{2}+l^{2}}\right) d n
$$

The result of the mix can then be calculated using

$$
\sigma^{2}=\sum_{l=2}^{n} g(l)^{2} P_{2-\mathrm{D}}(l)=325 \mu \mathrm{m}^{2},
$$

where the growth factors $g(l)$ are computed by modeling the implosion. ${ }^{13}$ Figure 14 shows both the $2-D$ power spectra and $g(l)$. The resultant mix region has a width of $\sigma=\sqrt{325 \mu \mathrm{m}^{2}}=18 \mu \mathrm{m}$.

The result of this calculation is that the mix region is too large by about a factor of four. Since the original surface roughness was about $2 \mu \mathrm{m}$ RMS, we need to start with a surface with roughness less that about $0.5 \mu \mathrm{m}$ RMS and a length scale of about $6 \mathrm{~mm}$.

\section{Conclusion}

The analysis of the photographs of D-T surfaces indicates that their surface roughness is about $2 \mu \mathrm{m}$ RMS, or about four times rougher than that needed for an NIF target. Results from $\mathrm{H}_{2}$ and $\mathrm{D}_{2}$ experiments indicate that somewhat better surfaces can be produced by forming the layer at a temperature very close to the triple point and using high heat fluxes through the hydrogen ice. When these surface finishes are scaled to the length scale of the NIF target, they are in the range of $0.5-1.0 \mu \mathrm{m}$ RMS. We believe that this RMS value is a manifestation of the film making process and not set by some fundamental property of hydrogen since we have had continued success in improving the surface finish. We believe that significant improvements in the surface finish of beta layered D-T are achievable by adding artificial heating to the interior of the capsule. One technique for doing this, in which a plasma discharge is generated by the gas inside the capsule dissipating heat, has been suggested by a Japanese group. ${ }^{14}$ We will study this process and are seeking other new ideas to generate heat flux through the hydrogen ice in an ICF capsule. 


\section{Notes and References}

1. T. P. Bernat, E. R. Mapoles, and J. J. Sanchez, "Temperature- and Age-Dependence of Redistribution Rates of Frozen DeuteriumTr'tium," ICF Quarterly Report, 1, (2) 57, (1993).

2. In this paper we discuss the scaling of the surface roughness for both the RMS as a function of the transverse length of the sample (RMS $\alpha L^{(x)}$, and the power spectrum as a function of mode frequency $\left[P(f) \sim 1 / f^{\gamma}\right]$. These scaling relations adequately describe most of our data and imply a self affine character which is expected from most dynamic models of nonequilibrium surface growth. These two scaling exponents are simply related as $\alpha=(\gamma-1) / 2$, for $1<\gamma<3$.

3. John G. Brisson, John C. Mester, and Isaac F. Silvera, Phys. Rev. B 44, 12453 (1991).

4. M. A. Paalanen and Y. Iye, Surface Scionce, 170, 80 (1986).

5. K. Kono, U. Albrecht, and P. Leiderer, I. Low Te'mp. Pluysics, 82, 279 (1991).

6. M. A. Paalanen and Y. Iye, Surface Science, 170, 80 (1986).
7. U. Albrecht, H. Dilger, P. Leiderer, and K. Kono, Physica B, 165-166, 841 (1990)

8. Oscar E. Vilches, J. Low T'mup. Phy/s., 89, 267 (1992).

9. P. Zeppenfeld, M. Bienfait, F. C. Liu, O. E. Vilches, and G. Coddens, I. Phys. Franci, 51, 1929 (1990).

10. J. H. Bruning, D. R. Herriott, J. E. Gallagher, D. P. Rosenfeld, A. D. White, and D. J. Brangaccio, Appl. Opt. 13, 2693 (1974).

11. When the applied heat flux is much smaller $\left(\sim 10 \mu \mathrm{W} / \mathrm{cm}^{2}\right)$ changing the temperature over this range produces severe crystal deformations.

12. S. W. Haan, Hydrodymamic Instabilitie's on ICF Capsule's, Lawrence Livermore National Laboratory, Livermore, CA, UCRL-jC-107592.

13. S. W. Haan, Lawrence Livermore National Laboratory, Livermore, CA, private communication (1993).

14. C. M. Chen, T. Norimatsu, Y. Tsuda, T. Yamanaka, and S. Nakai. "Redistribution of Solid Fuel in ICF Targets by Plasma," submitted to J. Vac. Sci. A. 


\title{
Large Aperture Sol-Gel Random Phase Plates FOR BEAM SMOOTHING ON NOVA
}

\author{
S. N. Dixit \\ I. M. Thomas \\ B. W. Woods \\ A. J. Morgan \\ M. A. Henesian \\ P. J. Wegner \\ H. T. Pozvell
}

\section{Introduction}

In recent -ears, laser beam smoothing has become an integral part of high-power, ICF laser systems worldwide.' A consensus is building in the ICF community that smooth laser beams are essential to controlling and possibly suppressing hydrodynamic and plasma instabilities although the physical mechanisms for this suppression are still being investigated. In directly driven targets, hydrodynamic instabilities seeded by the nonuniformities in the laser beam can destroy the symmetry of the implosicn. On the other hand, parametric instabilities in the plasma surrounding the capsule in indirect-drive systems can lead to energy loss and redistribution due to scattering and could therefore affect the coupling efficiency and drive asymmetry.

Techniques developed for smoothing laser beams can be divided into two categories: (1) spatial smoothing where the beam is broken up into a fine-scale spatial structure that the target can smooth by thermal conduction and (2) temporal smoothing where the fine scale structure changes rapidly, leading to a beam with time-averaged imoothness. In each of these methods, the plasma experiences a smooth beam as a result of spatial and/or temporal averaging. Spatial smoothing is achieved by the use of random phase plates ${ }^{2}$ (RPP) or lenslet arrays ${ }^{3}$, while temporal smoothing is implemented by smoothing by spectral dispersion ${ }^{4}$ (SSD), induced spatial incoherence 5 or amplified spontaneous emission ${ }^{\text {, }}$ techniques. We have recently implemented beam srnoothing on the Nova laser using RPPs and SSD." Time integrated smoothing levels of about $10 \%$ fintensity variance normalized to the average intensity) were achieved in averaging times of $10(1) \mathrm{ps}$. Experimental and theoretical investigations of the
Rayleigh-Taylor instability ${ }^{4,10}$ and theoretical investigations of filamentation ${ }^{11}$ in the presence of smooth beams have also been reported. Studies of the effects of smooth beams on stimulated Brilluoin and Raman scat. tering in laser produced piasmas are in progress. RPPs have also been used extensively on Nova for homogenizing the focal spot used for generating the $x$-ray backlighter beam.

Fabricating RPPs for use on Nova posed several unique challenges. To avoid damage to dowristream optical elements caused by the Fresnel diffraction from the edges of the phase plate elements, we chose to place the RPPs as the final optical element (which also acts as a debris shield) on the Nova beam. This required fabricating large aperture ( $80-\mathrm{cm}$ diam) RPPs. Additionally, the RPP coating must withstand high fluences in the visible $(527 \mathrm{~nm})$ and the ultraviolet $(351 \mathrm{~nm})$ wavelengtins, which are typically as high as $1.5 \mathrm{~J} / \mathrm{cm}^{2}$ in a $\sim 1 \mathrm{~ns}$ pulse with as much as 2:1 intensity modulations. Previously, RPPs have been fabricated by vapor deposition of a $\mathrm{SiO}_{2}$ layer ${ }^{12,13}$ or directly coating a photoresist ${ }^{14}$ to produce the relief pattern. Vapor deposited coatings are expensive and difficult to produce at large apertures, while the photoresist coatings suffer from low damage thresholds in the ultraviolet.

To overcome the cost and damage threshold limitations, we developed a novel sol-gel process for fabricating bi-level phase relief structures. ${ }^{15}$ The sol-gel coating was deposited from a liquid solution of an organosilicate polymer which was converted to silica after the alcohol carrier had evaporated. The sol-gel RPPs were prepared by selectively etching away areas of the slightly porous $(\sim 5 \%$ porosity) silicate coating layer. The coating process is similar to that used in preparing the antireflection (AR) coatings ${ }^{16}$ except that a polymeric silicate solution 
is used in RPP fabrication instead of a colloidal silicate suspension. This RPP fabrication procedure can easily be scaled to large aperture substrates and yields high quality products with excellent optical performance and high optical damage thresholds. To date, we have fabricated several bi-level RPPs of different aperture and element sizes for use in infrared, visible, and ultraviolet wavelengths. Table 1 lists the RPPs fabricated for use on Nova and other high-power laser facilities around the world.

\section{RPP Design Considerations}

A typical RPP used for ICF studies consists of a contiguous layout of regularly spaced elements randomly selected to be either "on" or "off"; the on elements impose a $\pi$-phase shift on the beam relative to the off elements (Fig. 1). RPP element shapes are typically hexagons, rectangles, and squares. As discussed elsewhere, ${ }^{8,15}$ the focal plane intensity distribution resulting from the focusing of light through a bi-level RPP consists of an overall envelope produced by the diffraction pattern of a single element, which is modulated by a fine-scale interference pattern due to contributions from various RPP elements. We have discussed, in detail, the properties of the far-field diffraction patterins for rectangular and hexagonal apertures in a recent fublication. ${ }^{15}$ Typically, the pattern has an Airy function-like shape with a central maximum of the size $\sim 2 \lambda f / h$ (zero to zero), where $\lambda$ denotes the laser wavelength, $f$ the lens focal length, and $h$ the RPP element size. Figure 2 illustrates the far-field intensity profiles for a regular- and a stretched-hexagonal aperture. The rak intensity at the center of the pattern is given by

$$
I_{\mathrm{max}}=I_{0}\left(\frac{A^{2}}{\lambda^{2} f^{2}}\right)
$$

TABLE 1. RIP's produced using the sol-gel fabrication process

\begin{tabular}{|c|c|c|c|c|}
\hline $\begin{array}{l}\text { Aperture } \\
\text { size }\end{array}$ & $\begin{array}{l}\text { RPP element } \\
\text { size and shape }\end{array}$ & $\begin{array}{c}\text { Optical } \\
\text { phase delay }\end{array}$ & $\begin{array}{c}\text { Operating } \\
\text { wavelength }\end{array}$ & Af lications \\
\hline $80 \mathrm{~cm}$ & 5-mm hexagomal & $\pi$ & $527 \mathrm{~nm}$ & Backlighter homogenization \\
\hline $80 \mathrm{~cm}$ & $7-\mathrm{mm}$ hexagenal & $\pi$ & $527 \mathrm{~nm}$ & Stimulated Raman and Brillouin scattering \\
\hline $8(1 \mathrm{~cm}$ & Stretched hexagona! & $\pi$ & $.351 \mathrm{~mm}$ & I'roduce a circular spot at oblique illumination \\
\hline $80 \mathrm{~cm}$ & $1.3-\mathrm{mm}$ square & $\pi$ & $.351 \mathrm{~nm}$ & Homogenize the plasma producing beam \\
\hline $20 \mathrm{~cm}$ & $\begin{array}{l}1.5-\mathrm{mm} \text { and } \\
3-\mathrm{mm} \text { hexagonal }\end{array}$ & $\begin{array}{l}\pi \text { and } \\
0.6 \pi\end{array}$ & $5.32 \mathrm{nni}$ & $\begin{array}{l}\text { Filamentation experiments at } \\
\text { Laboratoire LLLL, Ecole Polytechnique, } \\
\text { Palaiseau, France }\end{array}$ \\
\hline $201 \mathrm{~cm}$ & $3-\mathrm{mm}$ hexagunal & $\pi$ & $1053 \mathrm{~nm}$ & $\begin{array}{l}\text { Ion accoustic wave studies at I.LE, } \\
\text { Rochester, NY }\end{array}$ \\
\hline $13 \mathrm{~cm}$ & 1.t-mm hexagunal & $\pi$ & $11153 \mathrm{~nm}$ & $\begin{array}{l}\text { Beam homogenization on the (Octal laser } \\
\text { at Limeil, France }\end{array}$ \\
\hline $13 \mathrm{~cm}$ & 2-mm hexagromal & $\pi$ & $1115.3 \mathrm{~nm}$ & Beam homogenization on the Octal laser \\
\hline $13 \mathrm{~cm}$ & 1.t-mm hexagonal & $\pi$ & $.351 \mathrm{~mm}$ & Beam homogenization on the Octal laser \\
\hline $13 \mathrm{~cm}$ & 2-mm hevagonal & $\pi$ & $351 \mathrm{~nm}$ & Beam humogenization on the Octal laser \\
\hline
\end{tabular}

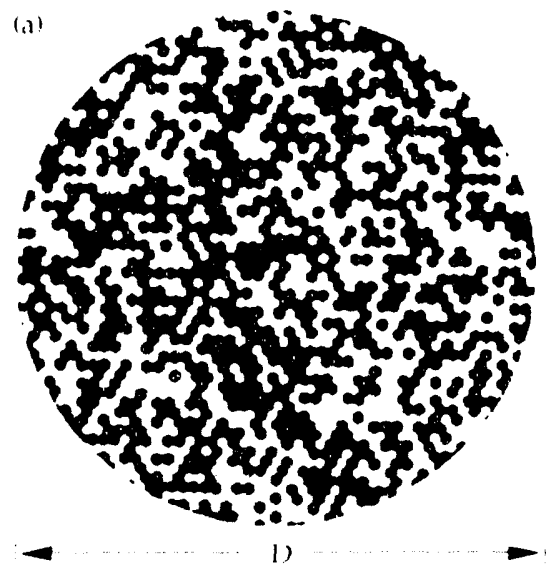

(b)

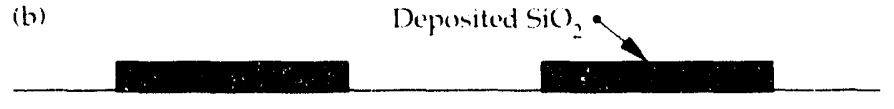

$\mathrm{SiO}_{2}$
Ficiurl: 1. (a)

Schematic lavout of an RPP consisting of randomly distributed on-off-phase plate elements. (b) A cross sectional view of a segment of an RI'? A relative optical phase delay of $\pi$ between the on- and the os: clements is achieved by selectively depositing a thin SiO laver. 
where $I_{0}$ is the incident intensity and $A$ is the area of a single RPP. If we assume that the envelopes due to various RPP elements add incoherently (neglecting the speckle pattern), then the peak intensity for the envelope is simply

$$
I_{\text {peak }}=N I_{\max }=N I_{0}\left(\frac{A^{2}}{\lambda^{2} f^{2}}\right)=P\left(\frac{A}{\lambda^{2} f^{2}}\right),
$$

where $N$ is the number of illuminated RPP elements and $P=N I_{0} A$ is the incident power. Detailed calculations including the speckle pattern confirm this scaling law when the speckle in the far-field pattern is smoothed out.

The desired RPP element size is determined by the required spot size and the peak intensity for a given input power in the laser. For example, to obtain a farfield spot with a peak intensity of $2 \times 10^{15} \mathrm{~W} / \mathrm{cm}^{2}$ for a 1-TW input power at $527 \mathrm{~nm}$, the RPP elements should have an area of $0.5 \mathrm{~cm}^{2}(f=3 \mathrm{~m})$. This would imply about $7-\mathrm{mm}$ square elements or about 7.6- $\mathrm{mm}$ (side-toside) regular hexagonal elements. The extent of the
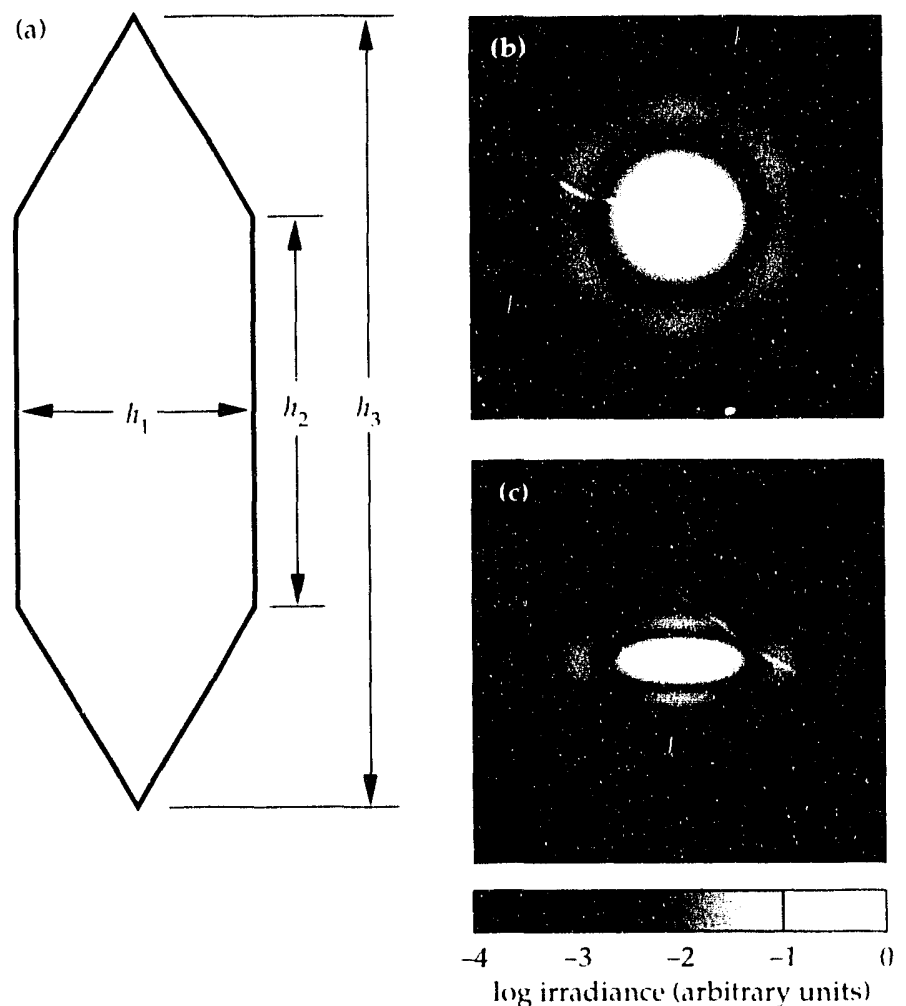

FigURE 2. (a) Parameterization of a stretched hexagonal aperture. Far-field diffraction patterns (b) for a regular hexagonal aperture $\left(h_{3}=2 h_{2}=2 h_{1} /, 3\right)$ and $(c)$ for a stretched hexagonal aperture with $h_{2}=2 h_{1}$ and $h_{3}=3 h_{1}$. The transverse distances for both case's are in units of $\lambda_{0} / h_{1}$ and the peak intensity is nomalized to 1 . central maximum would be about 450 or $480 \mu \mathrm{m}$, respectively. Note that whenever a large number of RPP elements contribute to the far-field pattern, the individual speckle intensities could be many times the envelope intensity because of the statistics of the resulting speckle pattern.

\section{Fabrication of Sol-Gel Phase Plates}

Conventionally, RPPs are prepared by evaporating silica in a vacuum onto a substrate that has been coated with photoresist. ${ }^{12,13} \mathrm{~A}$ suitable random design is patterned with a photomask in the photoresist. The development of the exposed photoresist leaves bare and coated areas on the substrate. A uniform layer of silica of precise thickness is then deposited on the substrate using conventional vapor deposition methods. The photoresist and the evaporated silica coating are chemically removed, leaving a pattern of silica pixels that corresponds to the positive image of the photomask. This is the RPP. It is important in this process to precisely control the thickness of the deposited silica and to ensure that the bare areas on the substrate (following the photoresist development) are extremely clean before silica vapor deposition so that a sharp pattern can be obtained. Working with Spectra Physics Optics Corp., ${ }^{12}$ we fabricated the first two $80-\mathrm{cm}$-diam RPPs using this process.

We have recently developed a new sol-gel process to fabricatc the RPPs. ${ }^{15}$ Figure 3 shows the main steps in this process. It differs from the vapor deposition method described above in that the whole substrate is first coated with a sol-gel silica layer which is then selectively etched off through a patterned photoresist layer that is deposited over the silica coating. The silica under the coated areas is protected from attack by the dilute HF etchant that removes the bare or unprotected silica. The patterned substrate is formed by removing the resist; the pattern left is the positive of the original on the photoresist and a negative of the photomask. The inhouse sol-gel process is cost effective and produces products with high damage threshold $\left(>8 \mathrm{~J} / \mathrm{cm}^{2}\right.$ at $532 \mathrm{~nm}$ and $>4 \mathrm{~J} / \mathrm{cm}^{2}$ at $351 \mathrm{~nm}$ for 10-ns pulses). Details of the fabrication process are described below.

\section{Silica Coating Deposition}

The first step in the sol-gel RPP fabrication is depositing a uniform layer of silica on the substrate. We found that substrates of varying sizes ( 5 to $80 \mathrm{~cm}$ diam) can be conveniently coated by pulling the dipped substrate out of a 200-1 tank of hydrolyzed ethyl silicate solution. A similar dip-coating method has been used extensively in our laboratory to prepare sol-gel AR coatings. ${ }^{16}$ About 
2001 of hydrolyzed ethyl silicate solution containing the equivalent of $10 \% \mathrm{SiO}_{2}$ is prepared directly in the coating tank by dissolving tetraethylsilicate $(25 \mathrm{~kg})$ in anhydrous ethyl alcohol $(189 \mathrm{~kg})$ and then slowly adding diluted hydrochloric acid ( $10 \mathrm{~g}$ in $19.4 \mathrm{~kg}$ water). The solution is continuously filtered and recirculated through a $1-\mu \mathrm{m}$ cartridge filter. A slight exotherm indicates that hydrolysis was occurring. After $30 \mathrm{~min}$, more tetraethylsilicate $(31 \mathrm{~kg})$ is slowly added over a period of another $30 \mathrm{~min}$. The solution is circulated and filtered for an additional $60 \mathrm{~min}$. It is then left sealed in the tank at room temperature for a period of at least two weeks prior to use to achieve the desired polymerization of the particulate silica.

Samples are dip-coated in the silicate solution in a clean environment at withdrawal rates varying from $4-8 \mathrm{~cm} / \mathrm{min}$, depending on the thickness requirement. (Slower pull rates give thinner coatings.) Since the viscosity of the sol-gel solution changes over a period of a few weeks, witness samples (up to $15 \mathrm{~cm}$ diam BK7) are pulled each time to determine the required rates. The silicate coated samples are dried at room temperature for $6 \mathrm{~h}$ and then recoated if necessary to produce additional thickness. They are then placed in a closed container over concentrated ammonium hydroxide solution at room temperature for a minimum of $24 \mathrm{~h}$ to thicken the coatings. The recoated samples are treated with ammonia vapor only after the final coating.

Depending on the reaction and cure conditions, coatings can range from quite porous to nearly fully dense. The dense films, which are strongest, are normally obtained by using acid-catalyzed solutions and by heating the substrate after the coating has air-dried. By exposing air-dried silica coatings made from an acid catalyzed solution to ammonia and water vapor for at least $24 \mathrm{~h}$ at room temperature, we found we can obtain coatings that are about $95 \%$ dense and require no additional heating. These coatings remain stable and unchanged for long period at room temperature, can be handled and cleaned like conventional optics, and can be etched in dilute hydrofluoric acid at a much faster rate than a fully dense substrate.

The thickness and the refractive index of the sol-gel coating were determined by measuring the transmission of the coated part in a spectrophotometer. The measured refractive index of the coating is $1.440 \pm 0.005$ at $527 \mathrm{~nm}$. Because the refractive index of the sol-gel silica coating is lower than that of the silica substrate (1.46 at $527 \mathrm{~nm}$ ), it is slightly antireflective. The optical thickness is determined from the positions of the transmission extrema. The optical path difference (OPD) at normal incidence between the coated and the uncoated regions is given by

$$
\mathrm{OPD}=\left(n_{c}-1\right) t
$$

where $n_{c}$ is the coating refractive index and $t$ is the thickness of the coating. To suppress the on-axis coherent spike, the OPD must be fairly close to $\lambda / 2$. Samples are usually prepared for use at either $351 \mathrm{~nm}$ or $527 \mathrm{~nm}$. Based on the measured value of the refractive index, these samples require thicknesses of 394 to $403 \mathrm{~nm}$ and 592 to $606 \mathrm{~nm}$, respectively. The former can be prepared with a single coating but the latter requires two coatings. For single coating samples (for UV applications), the pull rate is predetermined by the required thickness and the sol-gel viscosity. On the other hand, for coratings requiring multiple dips, the pull rates for later dips are adjusted to achieve the required thicknesses. We can routinely deposit coatings whose thickness is within a few percent of the desired value.

\section{Photoresist Coating, Development, and Etching}

The next step in the RPP fabrication is to selectively etch away the silica coating. This is accomplished by depositing a layer of photoresist, exposing it through a suitable photomask, developing it, and removing

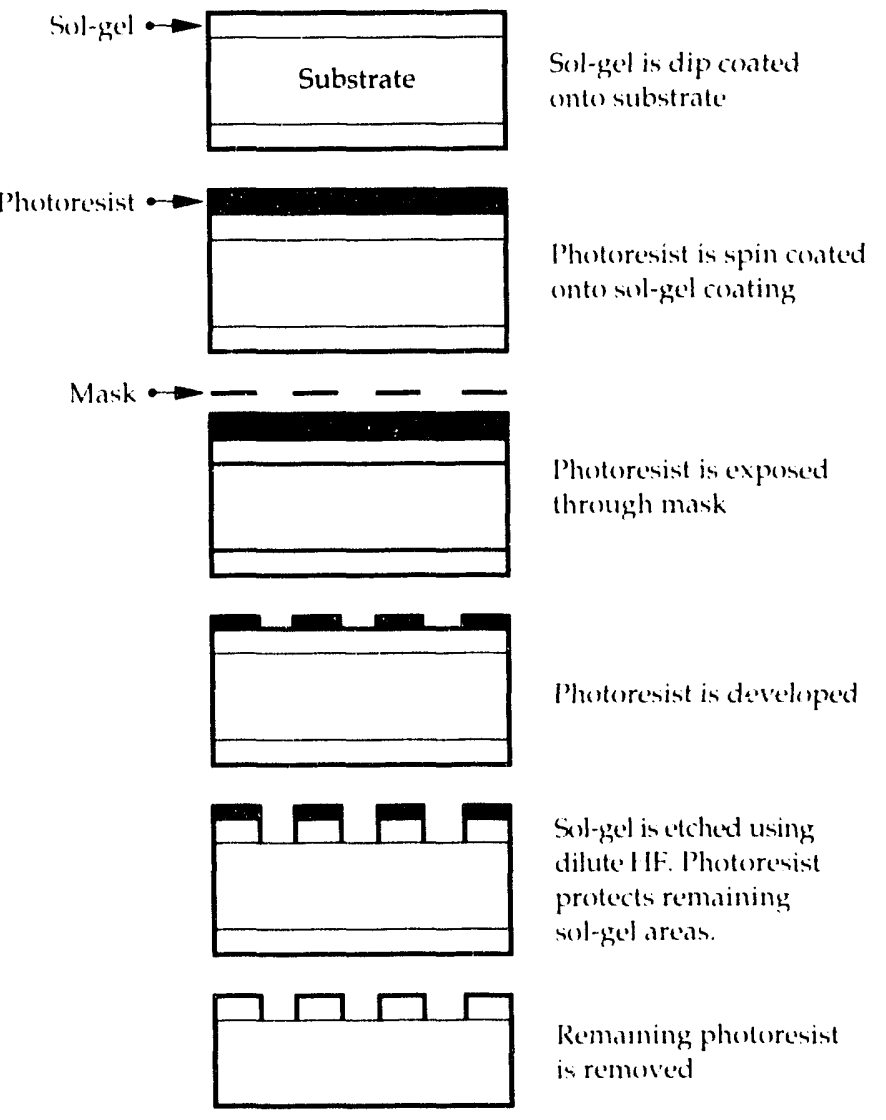

FICiURE 3. Schematic of the sol-gel RI'l' fabrication procedure. 
the sol-gel coating from the unprotected areas on the substrate. Although sol-gel silica coatings are easily deposited, there were several problems that had to be resolved in order to obtain high quality RPPs. We had to be certain (1) that the remaining resist pattern after development would protect the underlying silica layer from attack by the etchant and (2) that we could use an etchant concentration that would rapidly remove the slightly porous sol-gel silica and not affect either the photoresist layer or the dense underlying silica substrate. We also hoped to minimize the undercutting during the etching so that the pixels would remain sharply defined.

Because of the high cost of the photoresist, we applied the resist to the silicate coated substrate using a spin coating process rather than dip coating. To ensure good adhesion to the silicate, a thin layer of the coupling agent (Shipley C-50, hexamethyldisilazane in 2-ethoxyethyl acetate) is first applied to the sample at slow speed and allowed to dry for about $60 \mathrm{~s}$. This is followed by the photoresist (Shipley 1400-27) which is spun until dry to the touch. The coating speeds vary from approximately $1500 \mathrm{rpm}$ for the small $5-\mathrm{cm}$ samples to $300 \mathrm{rpm}$ for the large $80-\mathrm{cm}$ samples. The coated samples are finally dried in a forced air environment at $30^{\circ} \mathrm{C}$ for at least $16 \mathrm{~h}$. The photoresist coatings are $2-2.5 \mu \mathrm{m}$ thick.

The photoresist is then exposed through a photomask containing a black and white image of a random pattern of interlocking hexagonal, square, or rectangular RPP elements, as shown in Fig. 1. The mask is generated on a photographic film using a computer-controlled light pen stage with $25-\mu \mathrm{m}$ resolution. A random layout of the on-off elements is selected. The RPP mask is placed in contact with the resist and illuminated with UV light from germicidal lamps for approximately 70) $\mathrm{min}$. The resist is then developed using a dilute base (Shipley 354).

After development, we found that the unprotected sol-gel coating can be readily removed by etching in $1 \%$ aqueous $\mathrm{HF}$ solution for $4 \mathrm{~min}$. There is little effect on the substrate by the etchant solution in this period of time and photoresist conted silicate areas remain unetched. Sharp distinct silica pixels are obtained on the substrate after the remaining photoresist is washed away with acetone. Finally, the RPPs are AR coated with sol-gel porous silicalt before they are installed in the laser system.

\section{Phase Plate Characterization}

The RPP's were tested to find out how closely we had come to making a $\lambda / 2$ OPD between the on- and the off-elements. In an off-line optical test, ${ }^{15}$ we placed the RPP in a 30-cm-diam near-diffraction-limited beam from a CW argon ion laser and recorded the far-field image obtained with a 3-m focal length lens. The film image was digitized using a densitometer and processed on a computer.

If the OPD between the on- and the off- elements is different from $\lambda / 2$, an intense coherent spike can appear at the center of the far-field image. ${ }^{15}$ For a uniphase incident beam, the height of the coherent spike relative to the average intensity is given by

$$
\frac{\text { spike }}{\text { background }}=N \cos ^{2}\left(\frac{\delta}{2}\right) \text {, }
$$

where $N$ is the number of RPP elements illuminated and $\delta$ is the optical phase difference (OPD/ $\lambda$ ). When $N$ is large, $\delta$ must be very close to $\pi$ to suppress the coherent spike. We do observe coherent spikes in our off-line tests for many of the RPPs. Based on the measured height of the spike and Eq. (3), we estimate the OPD error to be less than $5 \%$. This is in reasonable agreement with topographic measurements of step height discussed below. An OPD error of a few percent would lead to a very intense coherent spike if the full 80-cm aperture RPP were illuminated with a coherent beam. However, as discussed below, beam aberrations on Nova reduce the effective coherence zone size, and hence minimize, and, in most cases, eliminate the coherent $s$ i ike. Thus a small OPD error is tolerable for Nova RPPs.

The thickness of the coating and the edge definition of the phase elements is determined by several methods. One method uses a high resolution phase-measuring laser interferometric microscope. The spatial resolution of the instrument with a $40 \times$ objective is $1 \mu \mathrm{m}$ with a vertical accuracy of $6 \mathrm{~nm}$. A second method uses a portable atomic force microscope (AFM). This instrument can scan areas as large as $100 \times 100 \mu \mathrm{m}$ and has vertical resolution on the atomic scale. Typical stylus pressures applied to the part are on the order of $10^{-8}$ Newtons. Both measurement methods yield the same physical step heights and edge definitions. The RPPs show a lateral edge definition of about $15 \mu \mathrm{m}$ and the step heights are typically within a few percent of the nominal.

We measured the laser damage thresholds of the RPPs at both 532 and $355 \mathrm{~nm}$ at a pulsewidth of $10 \mathrm{~ns}$ in our laser damage facility. The thresholds were nominally $30 \mathrm{~J} / \mathrm{cm}^{2}$ at $5.32 \mathrm{~nm}$ and $12 \mathrm{~J} / \mathrm{cm}^{2}$ at $355 \mathrm{~nm}$. Since the damage threshold scales as the square root of the pilse length, the damage thresholds are greater than $0 \mathrm{~J} / \mathrm{cm}^{2}$ at $532 \mathrm{~nm}$ and above $3.5 \mathrm{~J} / \mathrm{cm}^{2}$ at $351 \mathrm{~nm}$ for the typical 1-ns pulses on Nova. Under routine Nova operating conditions $\left(1 \mathrm{~J} / \mathrm{cm}^{2}\right.$ average fluence for $1-\mathrm{ns}$ pulses), we have not observed any optical damage associated with coating defects. 


\section{Performance of the RPPs on Nova}

The ultimate test of the usefulness of the RPPs is in their effectiveness in homogenizing the beam without producing a coherent spike on axis. The Nova beam is considerably aberrated because of large apertures and segmented optics. This leads to an approximately twenty times diffraction limited focal spot at the harmonic wavelengths. Figure 4(a) shows an example of the Nova farfield pattern. For comparison, a picture of the beam $4 \mathrm{~mm}$ past best focus is shown in Fig. 4(b) which has a spot size similar to that obtained with RPPs. This data as well as the data with the RPPs shown below was taken in a laser diagnostic station on one arm of the Nova laser that sampled the back-reflected light from a full apertur: 'eam splitter. ${ }^{17}$ It is clear from these pictures that both patterns are quite nonhomogeneous. Figure 4(b) is sufficiently far from focus that the characteristic near-field features of the beam (the central beam block and the segmentation from the frequency conversion array) are visible.

To homogenize this pattern, large aperture RPPs are placed in the Nova beam following the final focus lens, as shown in Fig. 5. The RPP's are installed at this position to avoid damage to downstream optics from near-field

(a)

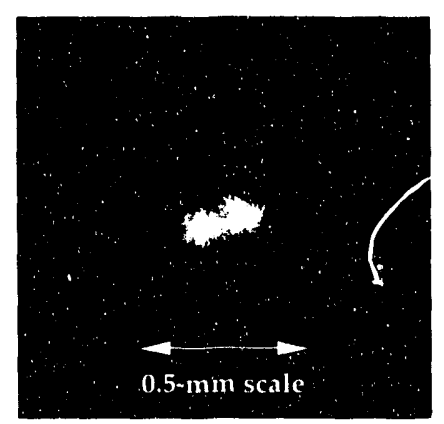

(b)

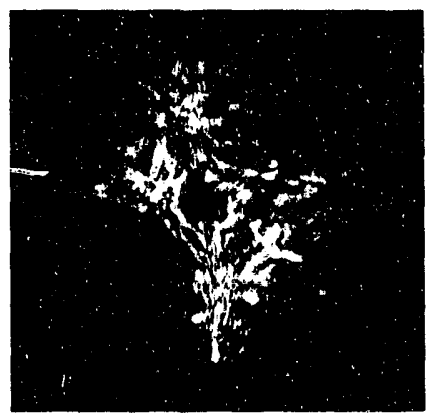

FIC CURI 4. Intensily distributions for the Nowa laser operating at $351 \mathrm{~mm}$ at (a) best focus and (b) in a plane 4 mm past bent focus. intensity modulations due to Fresnel diffraction around the phase step boundaries. ${ }^{18}$ The last optical element (RPl' or a plain $\mathrm{SiO}_{2}$ substrate) also acts as a debris shield to protect the focus lens against the target debris and is tilted at 7 to the beam propagation direction to prevent its back-reflections from hitting the frequency conversion array. The converging beam incident on the RPP introduces a $\pm 1 \%$ variation in the OPD across the Nova aperture. However, this error is not expected to lead to a coherent spike because of beam aberrations, as discussed below.

Figure 6 shows the far-field patterns obtained when the regular hexagonal, the square, and the stretched hexagonal RPP's are inserted in the Nova beam. The introduction of the RPP' in the beam completely washes out the Nova beam features shown in Fig. 4 and produces a homogeneous far-ficld pattern characteristic of a single RPP element. Notice, however, that to do so, the focal spot is necessarily increased in size. The clectric field in the focal plane is a convolution of the far-field patterns for the beam alone and for the RPP. The finescale speckle superposed on the far-field envelope of a single RPI element is evident.

We did not observe any coherent spike when the RPP's were fielded on Nova despite the OPD crrors in our RI'l's. The full aperture Nova beam (beam area

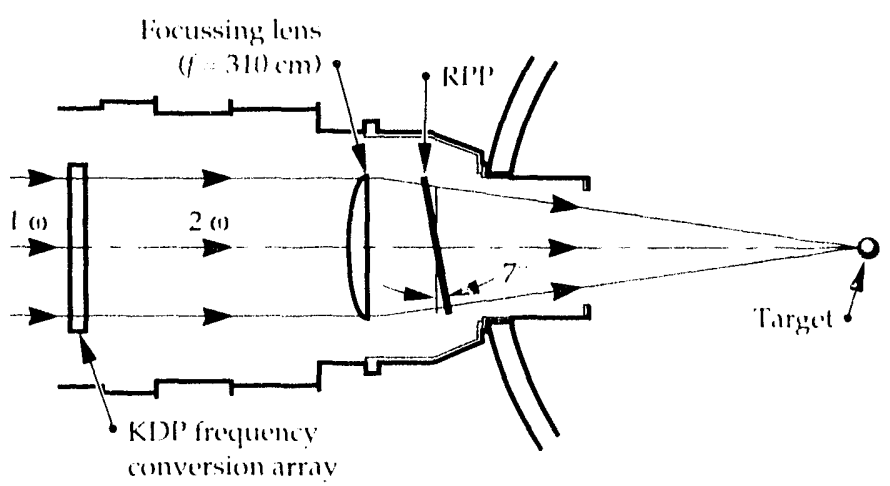

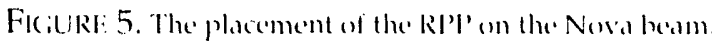

(.1) $7-1 \mathrm{~mm}$ hexagens $(527 \mathrm{~nm})$

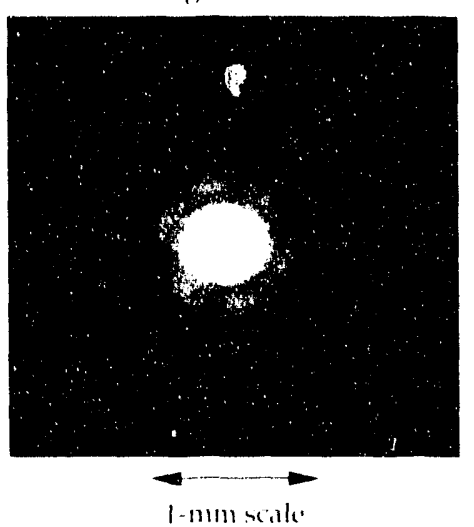

(b) $1.3-\mathrm{mm}$ hex.1g(0)s $(.351 \mathrm{~nm})$

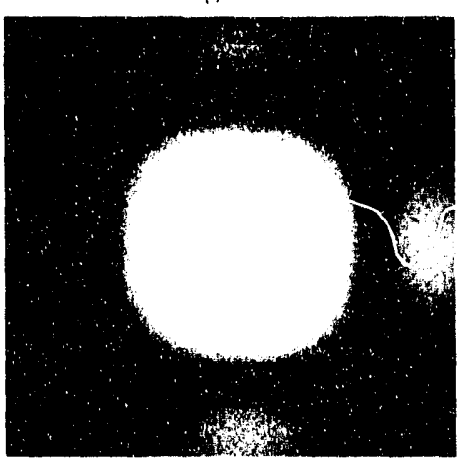

(c) Strothed hexagons $(351 \mathrm{~nm})$

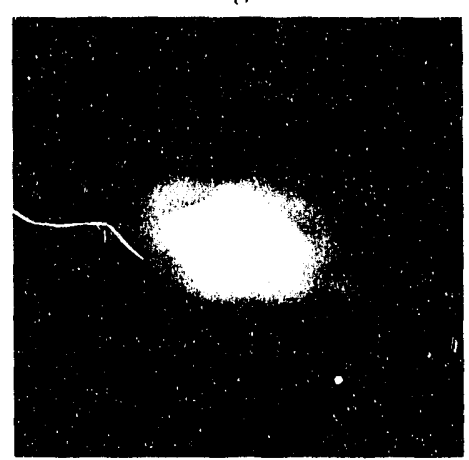

FIciURi: 6. Fartichd distributions on Novia with the Rll's inserled in the berm. Show'n against each picture are the size of the RI'l' element and the corresponding eperating wivelength. The sise of the streteded hexag (moll killelement was $h_{1} \cdots 3 \mathrm{~mm}, h_{2} \cdots 3.4 \mathrm{~mm}$ and $h_{3}=0.4 \mathrm{~mm}$. 
$\left.\sim 31(0) \mathrm{cm}^{2}\right)$ illuminates approximately 7,300 RPP elements on the $7-\mathrm{mm}$ hexagonal RPP $(18.3,4(0)$ for $1.3-\mathrm{mm}$ squares). Equation 4 implies that the OPD must be extremely close to $\lambda / 2$ to suppress the coherent spike in a uniphase beam. However, for nondiffraction-limited beams, the number of elements $N$ in Eq. (4) should be regarded as the number within a uniphase zone. Due to beam aberrations, the effective number of RIP' elements illuminated within a nominally uniphase zone on Nova (2-4 cm diam, estimated from the spot size at best focus) is much smaller than the total number of illuminated elements. This reduction in the number of coherently interfering RPP elements permits an increased tolerance on the deviation of the OPD from $\lambda / 2$. OPD errors of up to $5 \%$ from $\lambda / 2$ can be tolerated. For laser beams with a higher degree of phase uniformity, the allowed error is smaller.

\section{Conclusion}

We have presented the details of a new sol-gel procedure for fabricating large aperture bi-level RPPs. Several RPP's on substrates ranging from $15-80 \mathrm{~cm}$ in diameter were fabricated. Our sol-gel process is simple and produces a high optical quality, high optical damage threshold product. We presented experimental data illustrating the application of RPP's in homogenizing the far-field distribution of the Nova laser. The RIP's have proven very effective in homogenizing the focal plane intensity distribution of the Nova laser. The sol-gel RPP's are now routincly used in hydrodynamic and plasma instability experiments at LLNL and at other laboratories. Our ability to easily fabricate sol-gel RIP's has had a very positive impact on such experiments.

\section{Acknowledgments}

We wish to thank L. N. Manchester for assistance with the fabrication of the photegraphic mask, M. Baleoch for the AFM mensurements, and F. Rainer for the laser damage threshold measurements.

\section{Notes and References}

I. I. D. I indl, R. I. Mic rorv, and I: M. C amphell, "l'rogroms foward ignition and burn propagation in inertial ambinement

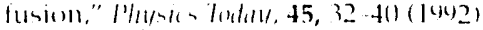

2. Y. Kato, K. Mima, N. Mivimaga, S. Arimaga, Y. Kitagawa,

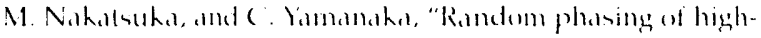
power lasers for uniform larged acceleration and plasma imsta-

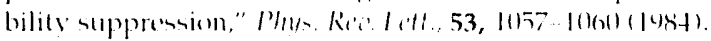

3. X. Dong, X. liang, / ( hen, W. Yu, and k. Ma, "L nitorm illa-

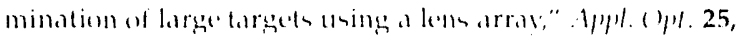
$377381(148 h)$
4. S. Skupsky, R. W. Short, T. Kesoler, k. S. Craxlon, S. Letering, and I. M. Soures, "lmprosed laser beam uniformity using the angular dispersion of frepuency modulated light," /. Appl. I'/ly/s. 66, $3456 \cdots 3462(1484)$.

5. R. II. I ehmberg and S. I? (Obensehain, "Lse of induced spatial incoherence for uniform illumination of haser fusion targets," ()

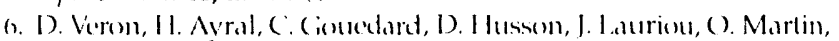
B. Meyer, M. Rostanong, and ( . Saluteret, "( )ptical spatial smowthing of Nd:glass laser beam," ()pl. (immm. 65, 42 40 (1988).

7. II. Nakano, K. Tsubakimolo, K. Yagi, T. Jitsamo, M. Notkalusha,

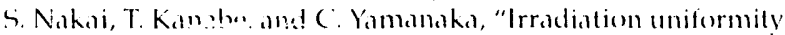
improvement be incoherence of amplitied spentaneous emis-

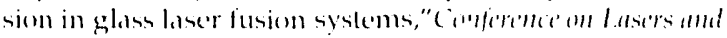

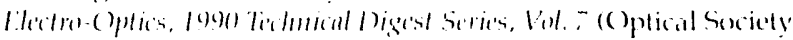
of America, Washingtom, D). (., CWF4s, gov(l) pp. $2801-282$.

8. 11. T. I'ow'dl, S. N. I)ixit, and M. A. I lem sian, "Berm

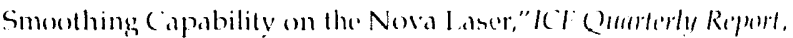

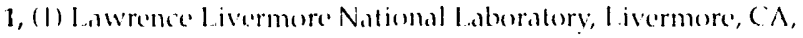
U( RLI-1.K-105821-4)1-1 (149(1) pp. 28-38.

9. S. C. Citendinning ot al.,"l.aser-Driven [Mamar kaylegh-Taylor

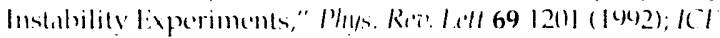

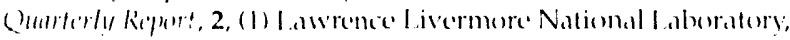

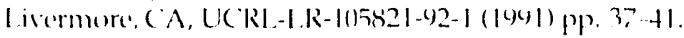

111. S. V. Weber al al. "later- Seded Modulation Cirowth on

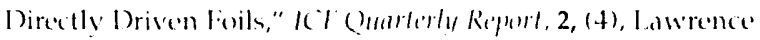
Livermore National Laboratorv, I ivermore. ('A, UCRI-I.R$115821-42-4(1993)$ Pr. 172-178.

11. K. 1.. Berger, B. I. Lasinshi, T. B. Kaiser, F. А. Williamm, A. B. langdon, and B. I. Cohen, "Three Dimensiomal Simulation of

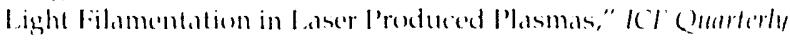
Kepent, 2, (3), I awrence I ivermore Natiomal I aboralory,

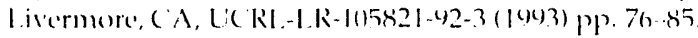

12. B. W. Wouds, I. M. Thomas, M. A. I lemesion, S. N. 1)ixit and 11. T. Powedl, "I arge Aperture (s(1)-cm Diameder) Phase Plates

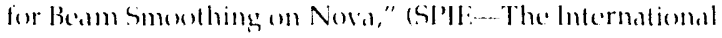

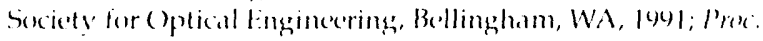
Sill 1410 ) pl. $47-54$

13. "OMl: is phase omversion with distributed phase plates," in

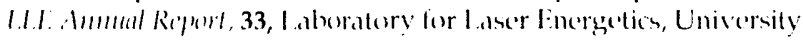

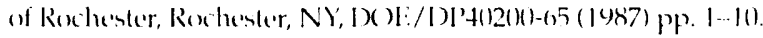

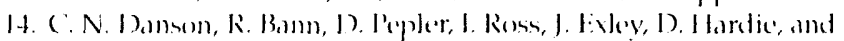
s. Sails, "I development af random phase plate smonthing fochmology:" Rutherford Appleton l ahoratory Ammal Report, RAl.-

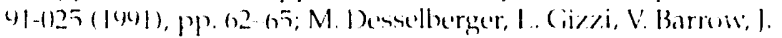

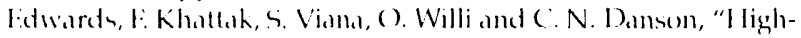
aspent-ratio line forus and plosma production using a random phase plate", Ayple (1) 31. 31, 3759-3766, (14092).

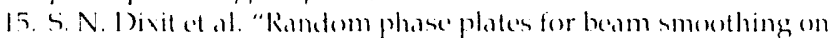

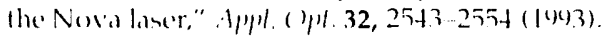

66. I. M. Thomas, "High laser damoge threshold porous silica

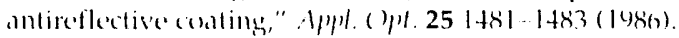

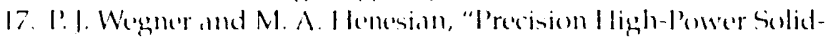

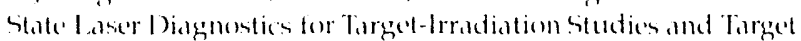
Ilane Irradiation Modelling," (Sill: The Intermational Sociedy

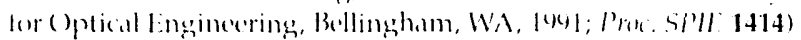
Pp. $162-17.4$.

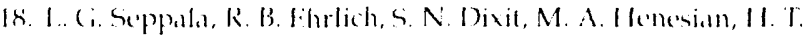

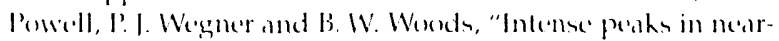

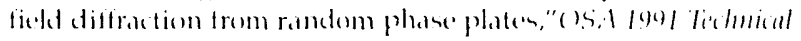

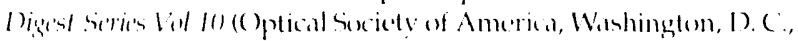
(WI41, |(x)1) 1). 27.4. 


\title{
NeUtron Time-OF-Flight ION Temperature Diagnostic For Nova
}

\author{
R. E. Chrien* \\ D. B. Harris** \\ M. D. Cable \\ M. B. Nelson \\ D. F. Simmons ${ }^{\dagger}$ \\ D. L. Holmberg ${ }^{t}$
}

\section{Introduction}

Neutron time-of-flight (TOF) detectors provide important information about the fuel-ion burn temperature in ICF targets. Conventional current-mode neutron TOF detectors measure the history of the total light output from many neu'ron interactions in a scintillator. These detectors provide accurate ion temperature data for neutron yields down to about $10^{111}$, but are limited at lower yields by the emission time of the scintillator, the response time of the detector, and the statistics of neutron scattering and light production in the scintillator.'

We have constructed and installed on Nova an ion temperature diagnostic, known as Tion ("tec-ion"), based on the timing of individual neutron interactions in many scintillators. This diagnostic is designed for indirect-drive Nova targets, whose neutron yields are typically too low for accurate ion temperature measurements with current-mode TOF detectors. The diagnos-tic measures the neutron arrival time distribution using an array of 1020 scintillator-photomultiplier detectors with about 1-ns time resolution and operates in the single-hit mode. ${ }^{2}$ The arrival-time distribution is constructed from 50 or more detector measurements. The diagnostic is located in a fan room west of the Nova target chamber, $27.10 \mathrm{~m}$ from chamber center.

The ion temperature $T_{i}$ is determined from the FWHM neutron energy spread $\Delta E_{\mathrm{n}}$ according to the relation ${ }^{3} \Delta E_{\mathrm{n}}=C_{\mathrm{dd}} T_{\mathrm{i}}^{1 / 2}$, where (for $T_{\mathrm{j}}$ and $\Delta E_{\mathrm{n}}$ both in $\mathrm{keV}) C_{\mathrm{dd}}=82.5$; for DT reactions the coefficient is $C_{\mathrm{dt}}=$ 177. The energy spread is related to the arrival-time

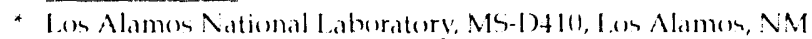
87545 (to whom correspondence should be addresened)

* Lom Alamos Natiomal laboratory

+ ECi\&fi Inergy Measuremente, Lats Vegan, NY
}

spread $\Delta t$ by $\Delta t / t=-(1 / 2) \Delta E_{\mathrm{n}} / E_{\mathrm{n}}$. Table 1 summarizes the quantities of interest. The raw data must be corrected to account for the system time resolution (about $1 \mathrm{~ns}$ ), the target burn time (about $100 \mathrm{ps}$ ), and the fuelion temperature profile.

In each channel, the neutron arrival times are detected using a photomultiplier tube (PMT) to observe the photons produced by recoil protons in a plastic scintillator. The recoil proton energy depends on the proton recoil angle $\theta$ through the kinematic relation $E_{\mathrm{p}}=E_{11} \cos ^{2} \theta$, where $\theta$ is measured with respect to the incident neutron direction. Since $n-p$ scattering is isotropic in the centerof-mass frame, the proton recoil energy distribution is uniform up to the neutron energy. The pulse height contains no information about the incident neutron energy, but can be useful for monitoring the system gain or in some pulse pile-up rejection methods.

The diagnostic covers DD neutron yields from $11^{7}$ to $2 \times 10^{4}$, a dynamic range of 200 . Part of this range is obtained by varying the number of channel hits in the array (from 5() to 5()()), and part is obtained by changing the scintillator volume (from 0.2 to $3.6 \mathrm{~cm}^{3}$ ). Operation at yields near $2 \times 10^{4}$ can provide overlap with results obtained from current-mode TOF detectors.

TABI.E 1. (haracteristico of D) and DT neutron times-offflight for Novid. Fight distance, $27.111 \mathrm{~m}$.

\begin{tabular}{lcc} 
Fusion reaction & DD & DT \\
\hline Inverne speed $(\mathrm{nm} / \mathrm{m})$ & 46.3 & 19.5 \\
Time of flight $(\mathrm{ns})$ & 1254 & 524 \\
Nf for $T, 1 \mathrm{keV}(\mathrm{ns})$ & 21.7 & 3.4 \\
1-M dintance $(\mathrm{cm})$ & 2.2 & 5.1 \\
\hline
\end{tabular}


Measurements at all accessible yields can be compared with estimates of $T_{i}$ obtained from first-hit analysis ${ }^{4}$ of DD neutron data from the Nova large neutron scintillator array (LaNSA). In first-hit analysis, only the earliest DD neutron arrivals are used to infer the ion temperature, on the assumption that the fuel ions have a spatially uniform and purely Maxwellian distribution.

The dynamic range of the array can be extended toward higher yield by operating in the multiple-hit regime. An acceptable fraction (10\%) of the channels will provide single-hit data even when $\alpha$, the average number of hits per channel, is equal to 3 . Thus a sixfold increase in dynamic range is possible, provided that channels affected by pulse pile-up can be reliably identified and rejected. Data reduction based on firsthit analysis ${ }^{4}$ (as in LaNSA) can also be considered.

\section{Hardware Description}

The detectors are enclosed in a cylindrical steel chamber (Fig. 1) that provides magnetic shielding for the PMTs. A total of 1020 PMTs are supported by fore- and back-planes made of black nylon to suppress neutron backscattering and stray light. Each PMT is pressed against a scintillator by a spring behind its base. A silicone pad is used to improve the optical coupling. Cesium-137 gamma sources are

FIciURE 1. Assembly drawing of the photomultiplier tube (PMT) chamber, showing scintillators, silicone coupling pads, PMTs, bases, and cables for the P'MT array.

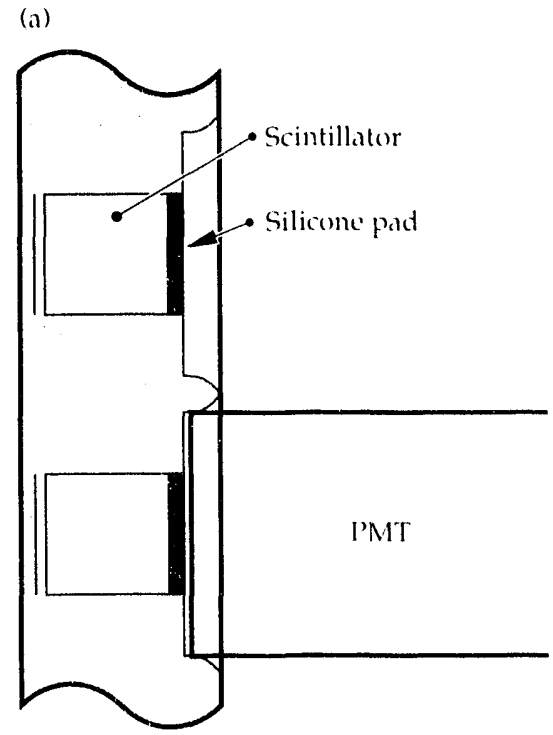

included for in situ calibration. The chamber is supported by a stand that allows it to be rotated for alignment or maintenance.

\section{Scintillator}

The scintillator volume is chosen to give neutron sensitivity appropriate to the yield predicted for a particular Nova shot. The scintillator thickness is restricted to no more than $2 \mathrm{~cm}$ to prevent the DD neutron transit time across the scintillator from degrading the time resolution. With this restriction, the number $\alpha$ of neutron interactions in each scintillator is given by

$$
\alpha=Y F \frac{A}{4 \pi R^{2}} \frac{x}{\lambda},
$$

where $Y$ is the neutron yield, $F$ is the neutron transmission factor (about (0.75), $A$ is the detector area, $R=27.1 \mathrm{~m}$ is the distance from the Nova target to the scintillator, $x$ is the detector thickness, and $\lambda$ is the neutron mean free path for elastic scattering $(7.7 \mathrm{~cm}$ at $2.5 \mathrm{MeV}$ and $28 \mathrm{~cm}$ at $14 \mathrm{MeV}$ ). For the minimum neutron yield of $10^{7}$ and for $\alpha=0.05$ (to obtain 50 measurements), the scintillator volume is $A x=3.6 \mathrm{~cm}^{3}$. For the maximum neutron yield of $2 \times 10^{4}$ and $\alpha=0.5$ (to obtain 500 measurements), the volume drops to $0.2 \mathrm{~cm}^{3}$.

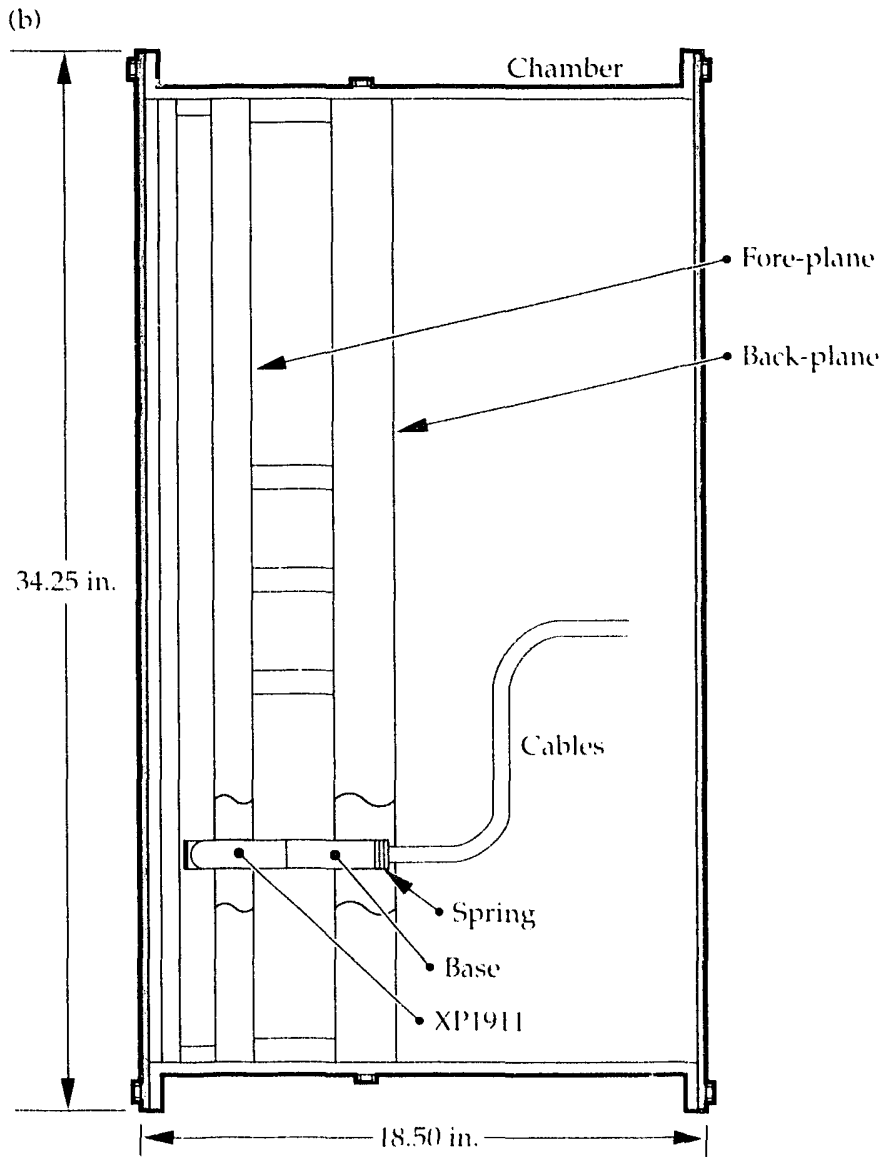


The scintillator is chosen to be relatively fast and bright, similar to BC400 or NE102A. The light output of these scintillators is about $64 \%$ that of anthracene. The light production in NE102A for electron energy deposition is 0.01 photons $/ \mathrm{eV}^{5}$ The production efficiency for protons in NE102 $\mathrm{A}^{6}$ can be obtained from the relation

$$
T_{\mathrm{e}}=0.95 T_{\mathrm{p}}-8.0\left[1-\exp \left(-0.10 T_{\mathrm{p}}^{0.90}\right)\right] \text {, }
$$

where $T_{\mathrm{p}}$ is the proton energy and $T_{\mathrm{e}}$ is the equivalent electron energy (both in $\mathrm{MeV}$ ). This relation is valid for $T_{\mathrm{p}} \geq 0.18 \mathrm{MeV}$. Figure 2 shows the dependence of light production on proton energy. The number of photons varies from 110 for a $0.25-\mathrm{MeV}$ recoil proton up to a maximum of 7433 for a full-energy recoil. For comparison, a full-energy recoil from DT neutrons produces 80,300 photons. A useful amplitude calibration is provided by $0.662-\mathrm{MeV}$ gammas from ${ }^{137} \mathrm{Cs}$, which produce a Compton edge at two-thirds of the recoil proton edge from DD neutrons.

Light coupling efficiency is estimated by comparing the pulse-height spectra from neutron or gamma interactions in a scintillator with the single photoelectron spectrum from the PMT. Various coupling methods were evaluated using $\mathrm{TiO}_{2}$-painted 1-cm-diam cylindrical scintillators and a 3-cm-diam end-viewing PMT. Coupling efficiency of about two-thirds is obtained when using optical coupling grease between the scintillator and the photocathode together with $\mathrm{TiO}_{2}$ paint on the uncoupled surfaces. Optical coupling grease cannot be used, however, because the scintillators must be changed between shots, so silicone coupling pads are used. These pads permit coupling efficiency close to that of coupling grease (about one-half). Bare coupling efficiency is around one-third.

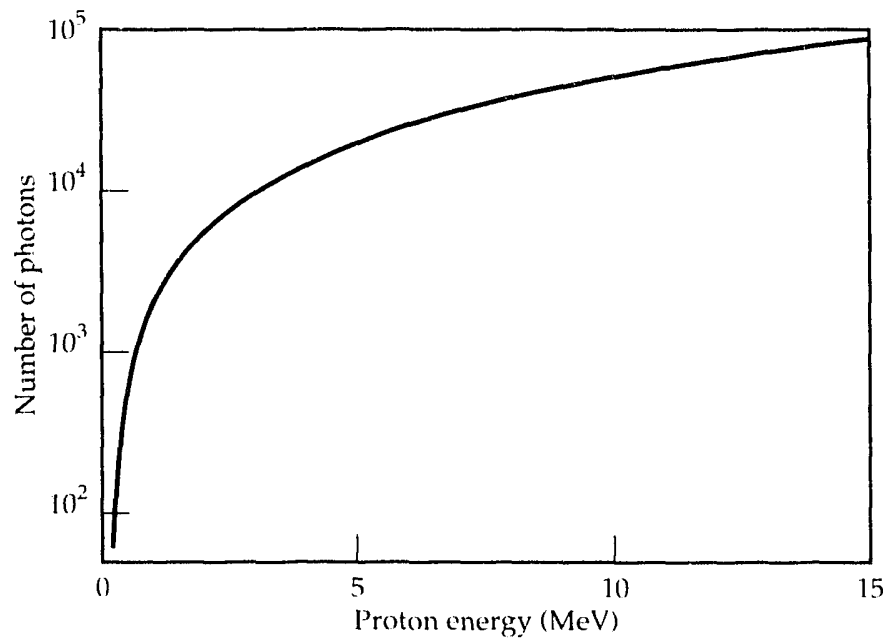

FIGURE 2. Light production by protons in BC40() plastic scintillator vs proton energy.

\section{Photomultiplier Tube}

The PMT converts scintillation photons into electrons and amplifies them in the electron multiplier. Bialkali photocathodes provide a good match for plastic scintillator emission spectra and have quantum efficiency of about $25 \%$. Thus 10 to 15 photoelectrons are generated by a $0.25-\mathrm{MeV}$ recoil proton. The multiplier structure must be a linear focused type to obtain good time resolution. Inexpensive side-viewing PMTs are unsuitable because of inefficient light coupling between scintillators and the recessed photocathode.

Time resolution of PMTs is quoted in terms of the single-photoelectron transit time spread (SPTTS).

These SPTTS values are 2 to 3 ns for PMTs of the type considered. The time resolution improves inversely as the square root of the number of photoelectrons; this dependence emphasizes the importance of efficient light coupling. An SPTTS of 3 ns or better is needed to obtain time resolution of $1 \mathrm{~ns}$ for DD measurements with as few as 10 photoelectrons. Still better time resolution is expected with brighter DT scintillations.

The anode output must be compatible with the discriminator threshold. Assuming a minimum of 10 photoelectrons, a gain of $10^{6}$, and a triangular anode pulse with a FWHM of $5 \mathrm{~ns}$, the peak anode current is $320 \mu \mathrm{A}$. This represents $16 \mathrm{mV}$ into a $50-\Omega$ load and provides a reasonable minimum voltage for the discriminators. In addition, the PMT output should remain linear up to the recoil proton edge (about $20 \mathrm{~mA}$ for DD neutrons).

A variety of small 10-stage end-viewing PMTs were considered. The Philips XP1911 19-mm tube was selected, primarily on the basis of low cost. This PMT provides the required gain (at a nominal voltage of $1600 \mathrm{~V}$ ) and linear current (up to $130 \mathrm{~mA}$ ).

The time resolution was measured by comparing the anode pulses from two XP1911 PMTs viewing the same scintillator (Fig. 3). Neutral density filters between the

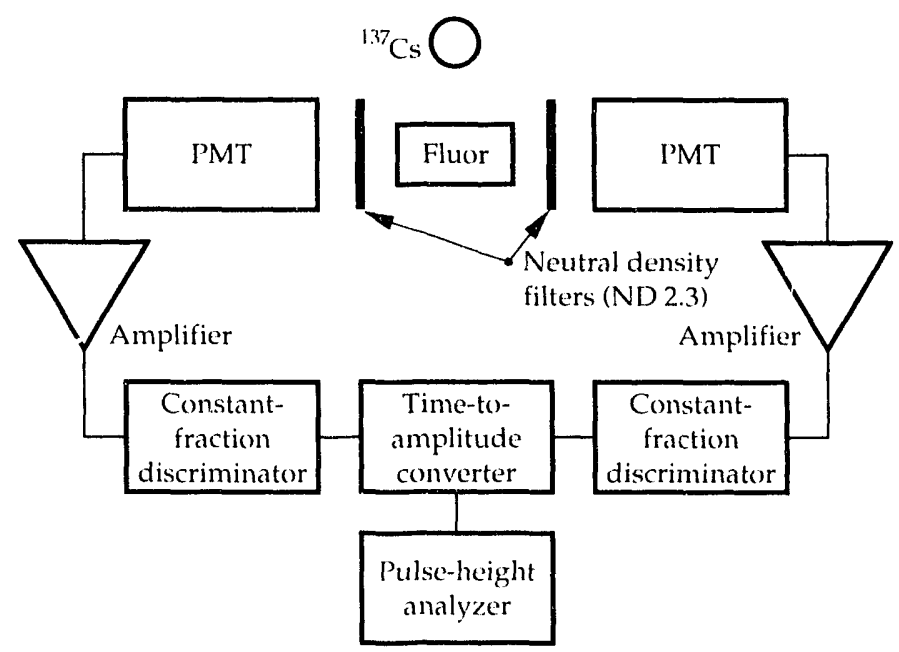

FIGURE 3. Electronics used for measuring single-photodectron transit-time spread by the two-photomultiplier-tube method. 
scintillator and each PMT provide an attenuation of $2(0)$, ensuring that each scintillation produces at most a single photoelectron pulse. Ortec VT120) linear preamplifiers provide an amplification of 10 for each anode signal. A 2()-ns coax line was used to delay one of the signals. Ortec 473A constant-fraction discriminators and an Ortec 567 time-to-amplitude converter were used to obtain the raw timing spectrum shown in Fig. 4 . The time spread $\Delta t$ can be expressed as $\Delta t^{2}=\Delta t_{\mathrm{A}}{ }^{2}+\Delta t_{\mathrm{B}}{ }^{2}+$ $\Delta t_{\text {scin }}{ }^{2}$, where $\Delta t_{A}$ and $\Delta t_{B}$ refer to the SPTTS of the two PMTs and $\Delta t_{\text {scin }}$ refers to the FWHM emission time of the scintillator (1.3 ns). For identical A and B channels, the SPTTS of each tube is 3.1 ns. Measurements with reduced neutral-density filtering confirm the expected improvement of time resolution witli light level.

The PMT voltage divider is conservatively designed for bleeder current equal to the average anode current rating of the tube. The manufacturer's recommended string for maximum linear current output is used. Capacitive stabilization of the latter dynode stages is provided to limit voltage changes to less than $1 \%$ for

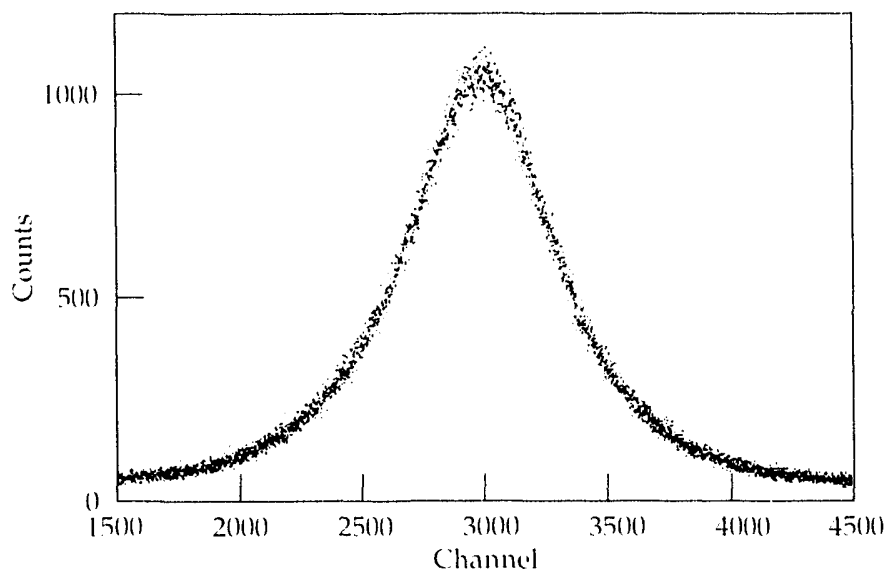

FICIURE 4. Transit-time spectrum obtained with the two-photomultiplier-tube method using XP1911 tubes and a BC+18 scintillator. The collibration factor is 155 channels per manosecond. The FWH IM corresponds lo $4.5 \mathrm{~ns}$.
10) full-energy proton recoil scintillations. Positive high voltage is supplied to the anode to permit the cathode region to be grounded. Anode output is capacitively coupled to the discriminator.

\section{Discriminator, ADC, TDC, and Data Acquisition}

The electronics for each channel (Fig. 5) consist of the PMT voltage divider base, a discriminator, a multiple-hit time-to-digital converter (TDC), and a gated charge-sensitive analog-to-digital converter (ADC). The multiple-hit capability of the TDC is valuable for observing the hard $x$ rays from indirect-drive Nova targets. These $x$ rays can be used to correct for timing differences betwern channels. ADCs can be valuable for recording the pulse height distribution of the recoil proton scintillations to monitor the overall health of the PMT array.

The anode signals are connected to novel constantfraction-like discriminators based on a simple $R C$ network and a fast voltage comparator. ${ }^{7}$ No walk correction is required with this discriminator. An updating output of 10 ns minimum duration is provided to allow pulse pile-up to be detected by the TDC. This discriminator has low power dissipation and can be made quite compact.

The discriminator provides a buffered version of the anode signal for connecting to a LeCroy $1885 \mathrm{~F} 15$-bit charge ADC. This is a 96-channel Fastbus module. The $1885 \mathrm{~F}$ can be gated for a time as short as $50 \mathrm{~ns}$ and can be used to measure the amplitude of the DD neutron pulses with a sensitivity of 50 () $\mathrm{CC}$ per count.

The discriminator output is comnected to LeCroy 1879 96-channel multihit TDC Fastbus modules. These are 2-ns TDCs with double-edge resolution of $10 \mathrm{~ns}$. Up to 16 edge timing measurements per channel can be obtained. For DT measurements, the TDCs can be upgraded to the faster LeCroy 1877 module. A DEC MicroVAX II computer is used to control and read out the Fastbus modules and to analyze the data.
FICiURE: 5. Electronics for a single detector channel.

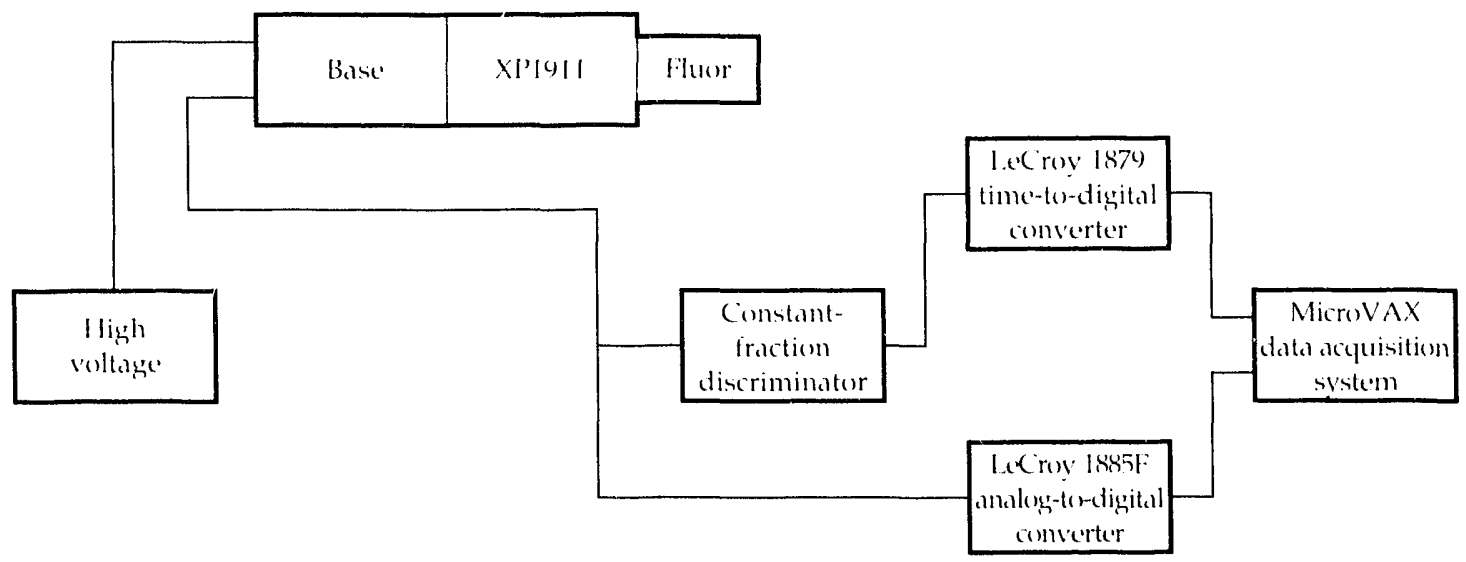


A CAMAC-based Starburst LSI-11 directs the control functions through a CAMAC-Fastbus interface. The software needed for this diagnostic was originally developed for the LaNSA array.

\section{Testing}

System pertormance is checked by monitoring the recorded data. Timing is routinely monitored by observing hard $x$ rays generated by indirect-drive targets. PMT failures are discovered by detecting a change in the divider string current, by the appearance of excessive noise, or by the absence of signal in a particular chamel for a series of shots. Discriminator and TDC problems are detected by comparing the average detection probability in each channel with the average detection probability in the whole array. An overall alignment check is performed by computing the average arrival time as a function of two orthogonal dimensions across the detector array. Individual channel timing variations are also detected by comparison with the average arrival time.

\section{Installation on Nova}

The diagnostic is installed in Nova Fan Room No. 3 (Rn. 1182) on a platform above one of the fan ducts (Fig. 6). The neutron flight path passes through a (0.66-mdiam opening in the concrete wall of the Nova 10-beam target hall. Neutrons and $x$ rays exit the target chamber through a $1.6-\mathrm{mm}$-thick aluminum vacuum window: traverse a modified section of Nova beam tube containing a 3.2-mm thickness of aluminum, and pass through a $1.6-\mathrm{mm}$-thick aluminum plate covering the hole in the wall. The cover of the array is $6.4 \mathrm{~mm}$ of type 1018 steel. Another $1.5 \mathrm{~mm}$ of aluminum in the plate holds the scintillators. It has proven necessary to add $12.7 \mathrm{~mm}$ of lead shielding just in front of the array cover to suppress the hard $x$ rays generated by indirect-drive Nova targets. Otherwise, photoclectrons produced by the $x$ rays cause the resistive bialkali photecathode to approach the first dynode potential in the PMT and thereby suppress the ability of the array to detect the subseyuent neutron arrivals.

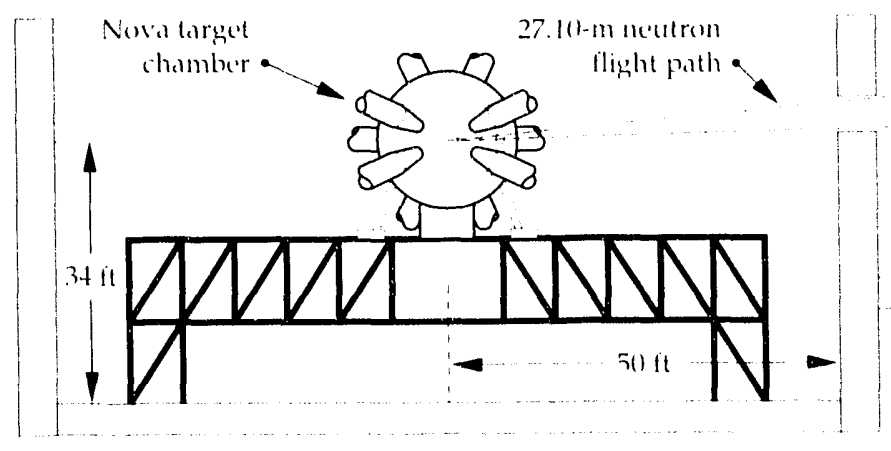

\section{Plan for Nova Experiments}

We are plamming two series of experiments on Nova using Tion. The first series is mainly for diagnostic checkout and cross-comparison; the second is to study the degradation of neutron yield with increasing capsule convergence ratio.

\section{Diagnostic Checkout Experiments}

Initial experiments with Tion will characterize the detector time response in the Nowa installation. These experiments will use direct-drive glass microballoon targets filled with $99 \%$ deuterium and $1 \%$ tritium, a fill that should produce about the same number of DT and DD neutron hits in the detector array. The ratio of DT and DD hits is approximately given by

$$
\frac{\alpha_{\mathrm{dt}}}{\alpha_{\mathrm{dd}}}=\frac{\lambda_{\mathrm{dd}}}{\lambda_{\mathrm{d} l}} \frac{2 n_{\mathrm{t}}}{n_{\mathrm{d}}} \frac{\langle\sigma i\rangle_{\mathrm{dt}}}{\langle\sigma\rangle_{\mathrm{dd}}},
$$

where $\lambda_{\text {dd }}$ and $\lambda_{\text {dt }}$ are the mean free pathe for elastic scattering by DD and DT neutrons, $n_{1}$ and $\|_{d}$ are the tritium and deuterium number densities, and $\left.\langle\sigma\rangle^{\prime}\right\rangle_{\text {dt }}$ and $\left\langle\sigma^{\prime}\right\rangle_{\text {dd }}$ are the DT and DD fusion reactivities. The factor of $2 \mathrm{in}$ the density ratio arises from the factor $1 / 2 \mathrm{in}$ the fusion reaction rate for identical DD reactants. The ratio of reactivities is a weak function of temperature," varying from 100 at $2 \mathrm{keV} \mathrm{to} 175$ at $10 \mathrm{keV}$. Thus for typical ion temperatures in direct-drive Nova targets, the expected ratio of DT to DD hits varies from 0.6 to 1 . The time spread of the DT neutron peak is influenced by both the detector time response and the neutron energy spread; the time spread of the DD peak is dominated by the temperature-induced energy spread. By obtaining both peaks in the same implesion, we can determine the detertor time response for the actual Nova conditions.

We have also planned experiments usmg indirectdrive targets to compare Tion results with LaNSA firsthit analysis at combinations of two temperatures (1 and $2 \mathrm{keV})$ and two DD neutron yields $\left(2 \times 10^{\mathrm{s}}\right.$ and $\left.1 \times 10^{6}\right)$. These conditions are obtained by varying wall thickness,

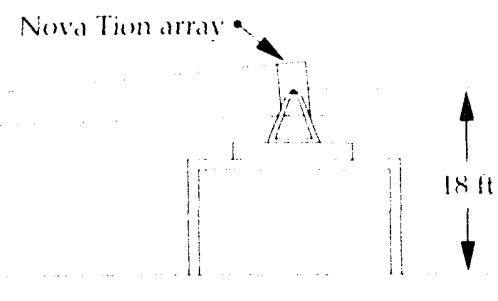

FIC,LRI 6. Justallation of the Tion dingmostic ini Noris. 
gas pressure, and hydrogen dilution in the target capsule. The comparison between Tion and LaNSA will help to validate the LaNSA first-hit $T_{\mathrm{i}}$ data at DD neutron yields below $10^{10}$, for which current-mode TOF detectors suffer large statistical uncertainties.

So far, LaNSA first-hit data have been interpreted under the assumption of uniform fuel ion temperature. At large values of $\alpha$, the LaNSA data might be expected to provide some information about the ion temperature profile. Comparisons between Tion and LaNSA will help to determine whether valuable $T_{\mathrm{i}}$ profile information can be obtained from LaNSA.

\section{High-Convergence Experiments}

These experiments will examine yield degradation in high-convergence implosions. Past experiments with both direct and indirect drive have shown significant yield degradation, relative to that calculated for clean one-dimensional implosions, for radial convergence ratios of interest in ICF applications. Two possible causes for this degradation are mix of the pusher material into the fuel or asymmetry of the implosion. We plan a series of targets with gradually increasing convergence ratio. The convergence ratio will be adjusted by varying the initial fuel pressure. The implosions will be diagnosed by measuring $T_{i}$, DD yield, fuel $p r$, shape of the imploded core, and spectroscopic emission of mix tracers. Yield degradation caused by asymmetry will be indicated by decreasing $T_{\mathrm{i}}$ and asymmetric core shape. On the other hand, a mix-dominated implosion is calculated to exhibit the same or somewhat higher measurement of $T_{\mathrm{i}}$ because the $T_{\mathrm{i}}$ profile is modified by cooling in the outer region of the fuel. Symmetric but smaller images are expected until mix permeates the entire fuel region; a large, weak image is then expected. Emission from a chlorine tracer added to the pusher material is another indicator of mix. These predicted differences between mix and asymmetry can be verified by deliberately inducing mix through capsule surface roughness or asymmetry through non-optimum laser pointing. By these methods, the relative contribution of mix and asymmetry can be assessed.

\section{Notes and References}

1. R. A. Lerche and B. A. Remington, "Detector Distance Selection for Neutron Time-of-Flight Temperature Measurements," Re't'. Sci. Instrum. b1, 3131 (199)()).

2. M. D. Cable, "Inertial Confinement Fusion Ion Temperature Measurements Using a Single-Hit Detector Array," I. Appl. P/11\%. 60. 3068 (1986).

3. H. Brysk, "Fusion Neutron Energies and Spectra," Plasmm Physics 15, 611 (1973).

4. R. A. Lerche, S. P. Hatchett, M. D. Cable, M. B. Nelson, and T. I. Murphy, "Plasma Temperatures from First-Hit Neutron Timeof-Flight Spectra," Re'c. Sci. Instrum. 63, 4877 (1)92).

5. C. F. Knoll, Ratiation Detection and Mensurements, 2nd ed. (Wiley, New York, 1989), Table 8-3.

6. R. Madev, F. M. Waterman, A. R. Baldwin, J. N. Knudson, J. D. Carlson, and J. Rapaport, "The Response of NE-228A, NE-228, NE-224, and NE-1112 Scintillators to Protons from 2.43 to 19.55 MeV," N id. Instrum. Methods 151, 445 (1978).

7. B. Turko and R. C. Smith, "A I'recision Timing Discriminator for H ligh Density Detector Systems," Lawrence Berkeley Laboratory, Berkeley, (A, LBL-306(1)2 (19991).

8. R. E. Chrien and J. D. Strachan, "Selective Fast Neutron Detector," Reir. Sci. Instrum. 51, 16.38 (1980). 


\title{
FACILITY REPORT JANUARY-APRIL 1993
}

\author{
G. Hermes
}

Nova Operations Group

Nova Experiments Group

Laser Science Group

During this quarter, Nova Operations fired a total of 277 system shots resulting in 345 experiments. These experiments were distributed among ICF experiments, Defense Sciences experiments, $X$-ray Laser experiments, Laser Sciences, and facility maintenance shots.

A series of experiments to measure beamline transmission and small signal gain was executed to update the Nova pulse shaping modeling code.

As part of the ongoing upgrade to the beam output diagnostic spools, the beamline eight chamber focus lens was exchanged with a new lens which has a new uniform $1 \%$ reflective coating. This coating provides a full aperture, spatially uniform signal for the output diagnostic clusters.

A variable vent valve was installed on the ten beam chamber. This valve, in conjunction with the new chamber vacuum control system, will allow the chamber to be cycled from vacuum to air to vacuum in only 25 minutes.

We have added smoothing by spatial dispersion (SSD) capability to beamline seven. We can now use SSD on beamline six or seven.
The first phase of installation and activation of the new target plane imager replacement (TPIR) has begun. The TPIR is used to view the reticule and beams at chamber center for target alignment. The new instrument is located off axis and is designed to be more reliable and quicker than the present TPI. After activation, the present on axis TPI will be removed to allow use of this premium location for other target diagnostics.

A significant portion of the installation and activation of the Precision Nova output diagnostic spools has been completed this quarter. The remainder of the beamlines are scheduled for completion by mid-May.

We are testing a Teflon-coated debris shield on the ten beam chamber (BL-7). This debris shield has been in place for several weeks and has suffered no significant damage. The Teflon coating should prove to be more durable over time than the sol-gel coating currently used.

We started moving into the Petawatt Lab this quarter and are beginning the installation of the optical tables and components. The Petawatt Lab will remain separate from the Nova system for several more quarters. 


\section{Publications}

\section{A}

Albritton, ]. R., and Liberman, D. A., On the Distribution of Boumd la'els of lons in Dense Plasmas: The Plasma Polarization Shift, Lawreace Livermore National Laboratory, Livernore, CA, UCRL-JC-111911 (1443). Prepared for I. Quant. Sprectruscopy \& Radiatioc Transfer.

Amendt, P., London R., and Strauss, M., Optimization Study of X'-Ray Laver Collerince in Ni-Like Ta, Lawrence Livermore National Laboratory, Livermore, CA, UCRL-KC-11(K)84 REP' (1993). Prepared for 3rt Internatural Collonumum on X-Ruy Lase,s, Schliersee, Cicmany, May 18-22, 1402 (Inst. Phys Cont. Ser. 125, 5).

Amendt, I'. A., Eder, D. C., Rosen, M. D., Penetrante, B. M., and London, R. A., Dimonstration De'signs for Optical-Field-lenized Plasma X-Ruy Lasers, Lawrenoe Livermore National Laboratory, Livermore, CA, LCRL-JC112424 (1 14.3). Prepared for 1993 SPIE Short-Pulse High-Intensity Lisers: and Appla thens II, Los Angeles, CA, January 21-22, 1993.

Atherton. 1., ()ptics Mimufacturing Derelopmerat for the National Isnition Facility (NIF), Lawrence Liveriaure National Laboratory, Livermore, CA. LCRL-MI-113073 (1993).

\section{B}

Barmard, J. J. Caporaso, G. J., and Yu, S. S., Ome Dimensional Simulations of Transients in Heney lon Injectors, Lawrence Livermore National Laboratory, Livermore, CA, UCRL-JC-112343 ABS (199.3). Prepared for PAC 93, Washington, DC, May 17-20, 1993.

Barnard, I. I., Deadrick, F., Griffitv, L. V.. Grote, D. P., Friedman, A., Kirbie, H. C., Neil, V. K., Newton, M. A., and Paul, A. C., Recirculating

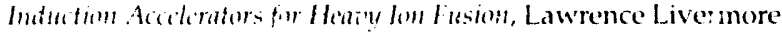
Nistional Laboratory, Livermore, CA, LCRL-JC-113284 ABS (1993).

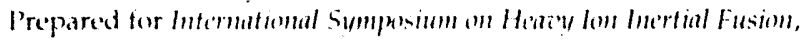
Frascati, Itals: May 25-28, 1943

Barnard, I. I. Miller, J. and Haber, I., Emittance Grouth in Displaced.

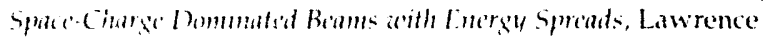
Livermore National Laboratery, Livermore, (A, LCRL-JC-112341) ABS (1443). Prepared for PAC 43. Washington, DC, May 17-20, 1493.

Bermat, T. P', Mapoles, E. R., and Collins, C., Surfaci Characteristics of

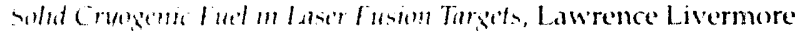
Cational Laboratory, l wermore, (A, LCRL-JC-1131150) ABS (1993).

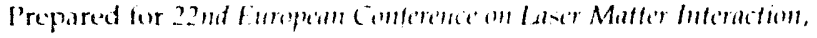
Paris, France, Mav 11-14, 1493
Bernat, T. P., McEachern, R., Wallace, R. I., and Letts, S. A., Control and Minsureme'nt of Surface Morphologies of ICF Capsule's, Lawrence Livermore National Laboratory, Livermore, CA, UCRL-JC-113049 ABS (1993). Prepared for 22nd Europian Conference on Laser Matter Interaction, Paris, France, May 10-14, 1993.

Bibeau, C., Payne, S. A., and Powell, H. T., Direct Mensureme'nts of the Terminal-Le'vel Lifetime for Nd ${ }^{3+}$-Doped Laser Mate'rials, Lawrence Livermore National Laboratory, Livermore, CA, UCRL-MI-111853 (1993). Prepared for Atranced Solid Statc Lasers, New Orleans, LA, February 1-3, 1993

\section{C}

Caird, J. A., Murray, J. E., and Powell, H. T., Precision Nora Project at II.NL, Lawrence Livermore National Laboratory, Livermore, CA, UCRL-JC-113057 ABS (1993). Prepared for 22nd European Conferenc' (1) Laser Matter Interactiem. Paris, France, May 10-14, 1993.

Caird, J. A., and Powell, H. T., Laser Improvements for the Precision Noria Projuct, Lawrence Livermore National Laboratory, Livermore, CA, UCRL-MI-105584, Rev: 1 (1993). Prepared for L.S. DOE Inertial Fusion Division, Germantown, MD, February 4, 1993.

Callahan, D. A., Langdon, A. B., Haber, I., and Friedman, A., Longitudinal Beam Dynamics for Heany Ion Fusion, Lawrence Livermore National Laboratory, íivermore, CA, UCRL-JC-112341 ABS (1993). Prepared for PAC 9.3, Washington, DC, May 17-20, 1993.

Callahan, D. A., Langdon, A. B., Haber, I., and Friedman, A., Longitutinal Berm Dymamics in Heary lon Husion Driser Beams, Lawrence Livermore National Laboratory, Livermore, CA, UCRL-JC-113229 ABS (1993). Prepared for 1993 IEEE Internutional Conference' on Plasma Scilnce, Vancouver, British Columbia, Canada, June 7-9, 1993.

Callahan D. A., Langdon, A. B., Haber, 1., and Friedman, A., Lemgitudinal Be'am Dymumics for Hen'y lon Fusion Using WARP RZ, Lawrence Livermore National Laboratory, Livermore, CA, UCRLMI-112247 (1993). Prepared for CAP9.3 Pleasanton, CA, February $23-26,1943$.

Compbell, E. M., The Physics of Me'ga-loule, Large'-Scale and Ultrafast Short-Siale Laser. Plasmats, Lawrence Livermore National Laboratory, Livermure, CA, UCRL-JC-107981 REP' (1993); Phys. Fluid B, 4, (November 1942).

Campbell, E. M., Inertial Confincme'nt Fusion (ICF) Program at Lairence Lizermore National Laboratory (LL.NL), Lawrence Livermore Natiomal l ahoratury. I ivermore. CA. UCRL-IC-113071 ABS (1993). I'repared for 22ud Europeran Conference' on Lasier Matter Interaction, Paris, France, May 10-14, 1993. 
Camphell, E. M., KF at IIINL and Ultrm-High Intensily lasers, Lawrence Livermore National Laboratory, Livermore, CA, UCRLMI-11.3072 (1993). Prepared for LI.NI/Commmontersolth of Independent State's Workshop, Livermore, CA, February 8, 1993.

Campbell, E. M., Hugan, W. J., and Lowdermilk, W. H., Norin Lpyrade Mission and Design, Lawrence Livermore National Laboratory, Livermore, CA, UCRL.JC-1101135 (1993). Prepared for

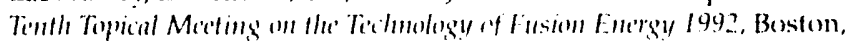
MA, June 7-12, 1942

Caporaso, (i. J., and Barnard, I. J., Analysits of Berom Loded:ng in Cletrostatic Cohumens, Lawrence Livermore National Laboratory, Livermore, CA. UC RL-JC-112.342 ABS (194.3). Prepared for PAC 9.3, Washington, DC, May 17-20, 1993.

Chen, X. M., Studu of Thermal Hydrandic and Kinetic Phe'nement in

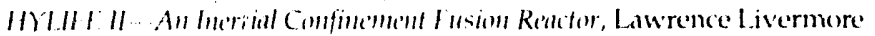
National l aboratory, Livermore, (A, UCRL-ID)-112698 (199.3).

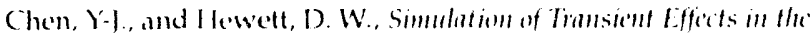

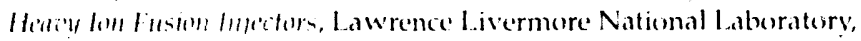
livermore, ( A, I (RL-JC-112344 ABS (1493). Prepared for BAC 93, Washington, 1) , May 17-20, 1493.

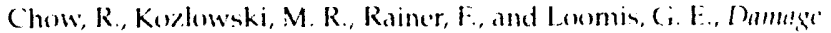

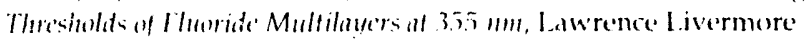
National Laboratory, Livermore, (A, LC RI_-KC-111482 (1943).

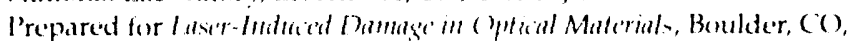
October 27-30, 1492.

Collins, C. W., Mapoles, F. R., and Lnites, W (i, Momstiy Proffile of Thich H, Lilmi, Lawrence Livermore National I aboratory, I.jermore,

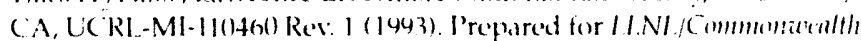

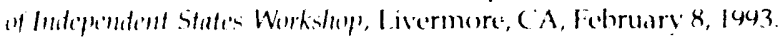

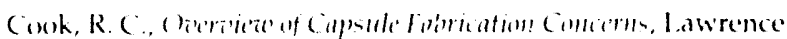
Livermore National Laboratory, Livermore, (A. UC RL-MI-113225

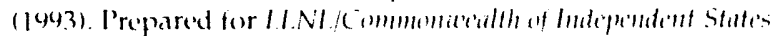
Workshep, Livermore, CA, fehruary 8, 1943

Cook, R. C., ()verturt, (;. F., L.elts, S. A., Kong, F. M., and Hacndler,

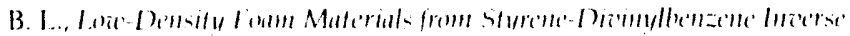

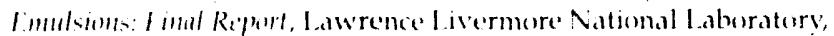
Livermore, ( A, LCRI-LR-11192117 (1943).

Correll, D. L., and (amphell, E. M., 1991 KC Amminl Report, Lawrence Livermore National Laboratory, Livermore, CA, UCRI.I.R-11:5R2(1)-4) (194.3)

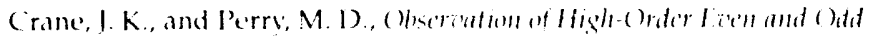

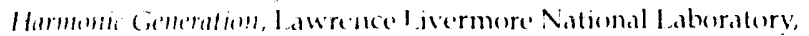

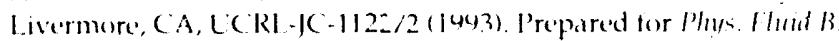

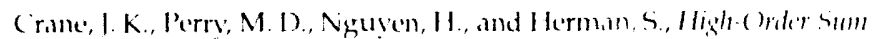

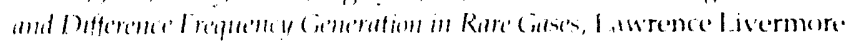

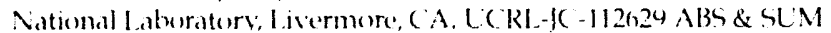

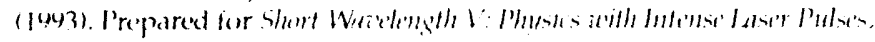

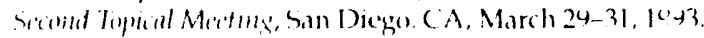

Crame, I. K., Perrs. M. D., Nguyen, H., Herman, S., falcome, R.,

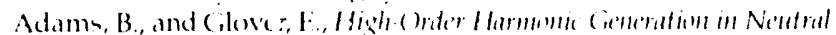

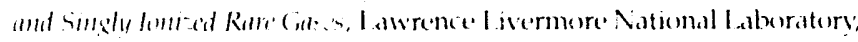

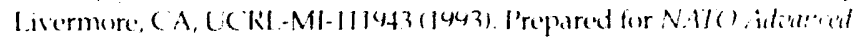

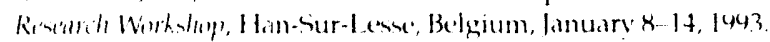

\section{D}

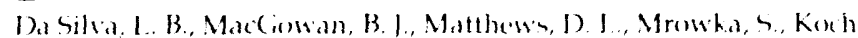

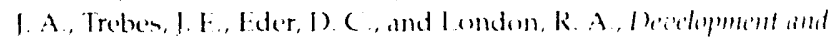

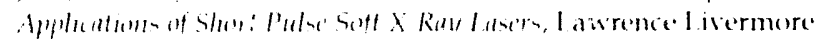

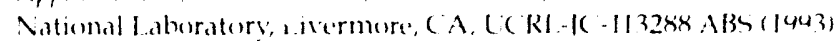

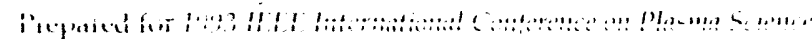
Vancourer, Britioh Columbia, Canda, fune $7-4,1493$.
Da Silva, L. B., Trebes, J. E., Kuch, J. A., Mackowan, B. J., Mrowka, S., Matthe'ws, D. D., Barbee, T. W., Balhorn, R., and Gray, J., Imaging

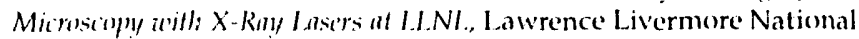
Laboratory, Livermore, CA, LCRL-JC-1118011 REP (1943). Prepared

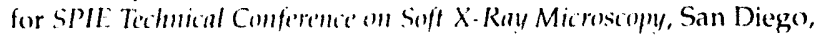
(A, July 19-24, 1992.

Dahlburg, J. P., Gardner, J. H., Doolen, G. D., and Haan, S. W., Effe't

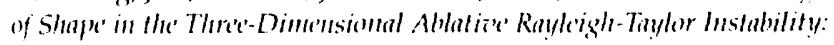
Multimode' l'erturbutions, Lawrence Livernore National Laboratory, Livermore, CA, UCRL-JC-112933 ABS (1993). Prepared for 4 th International Workshop on the Physics of Compressible Tirbuld Mixing, Cambridge, England, March 29-A pril 1, 1993.

Darrow, C. B., Ne'u, Physical Mechumismls in LItra-Intense Laser-Plasma Interations, Lawrence Livermore National Laboratory, Livermore, CA UCRL-JC-113041 ABS (1993). I'repared for Intermational Wintersichool and Workshop (m) Phismu Physics, Pich, Austria, February 22-27, 1493.

Davis, J. I., Campbell, E. M., Storm, E. K., and Lindl, J. D. Imertial Confine'menl Fusim, Lawrence Livermore National Laboratory, Livermore, CA, UCRL-JC-111721. Prepared for Modern Physics for Sicintists and linginests.

De Cirost, J. S., Estabrook, K. C., Mizun , K., Drake, R. P., K.ruer, W. L., and Cameron, S. M., Distributed Alsorplionn and luhibited Hent Trimingerf, Lawrence Livermore National Laboratory, Livermore, CA, UCRL.-KC-1119477 (1993). P'repared for 1011/ Workshop on Lasi'r Plasma-

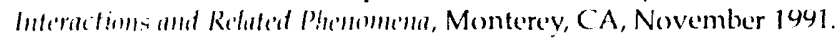

De Yoreo, J. J., Land, T. A., and Dair, B., Atomic Force Microsiony Study of Cimeth l Dymumics in KIl, Livermore Notional Laboratory, Livermore, CA, UCRI.JC-113148 ABS (1493). Prepared for Ninth American Comference on ( rystal Groneth, Baltimore, MD), August 1-6, 1993.

Deford, J. F. Calculation of Woke Potentials in Conities Comtaining

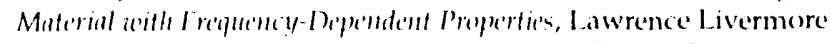
National Laboratory, Livermore, CA, UCRL.-MI-112262 (1943). Prepared for CAP93, l'leasanton, (A, February 23-26, 1493.

Deford. I. F, Hartung, W., and Moffat, D., Compuling Impedame' and

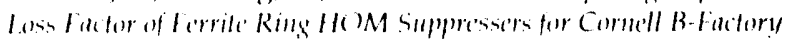
Superomductime Rl Cority, Lawrence Lisermore National Laboratory, l.ivermore, (A, LCRL-K -112374 ABS (1993). Prepared for PAC 93, Warhington, 1). May 17-20, 1493.

Deford, J. F., and Kozlowski, M. R., Medeling of llectric-licht Inhanciment at Nodular Defots in Dedectric Mirror Contings, Lawrence livermore National Laboratory, Livermore, CA, UCRL-JC-111462

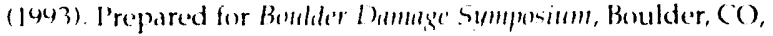
()ctober 28-30), 1492.

Deford, J. F, Madren, N., Rvine, R., Kodene, (i., and Shang, C. C.,

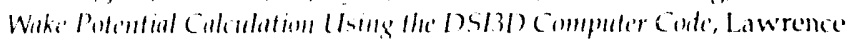
Livermore National Laboratory, Livermore, CA, UCRL-JC-112.376 ABS (1943). P'repared for BA 93, Washington, DC: May 17-21), 1993.

Deloach, 1. D. Payne, S. A., Kway, W. L., smith, L. K., Krupke, W. F.,

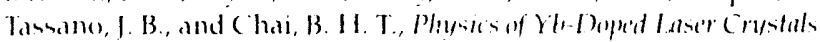
with the Apatile structuri, Lawrence livermore Notional Laboratory,

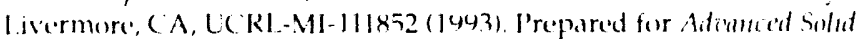
State laber, New Orleans, I.A, lebruary 1-3, 1443.

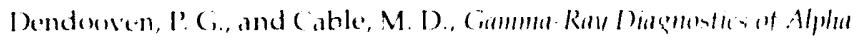

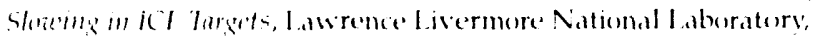

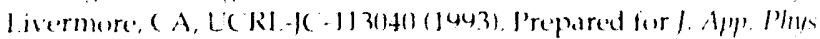

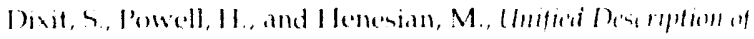

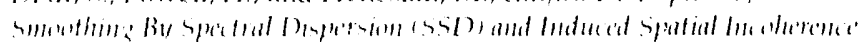
1/SL, Livermore National L aboratorv Livermore, CA, LCRI - MI-

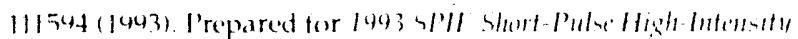

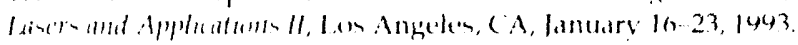




\section{$\mathbf{E}$}

Eastman Kodak, Summary of the Norn Upgrad' Baseline Cost Analysis, Lawrence Livermore National Laboratory, Livermore, CA, UCRLCR-11)268 (1993).

Ebbers, C. A., De Yoreo, J. J., Steiner, B., Rek, Z., and Zaitseva, N., Sources of luternal Struin in Mixed Crystals of $K\left(D_{1} H_{1}-X\right)_{2} \mathrm{PO}_{4}$ Lawrence Livermore National Laboratory, Livermore, CA, UCRL-JC113046 ABS (1993). Prepared for Ninth American Conferenci' on Crystal Growth, Baltimore, MD, August 1-6, 1993.

Eder, D. C., Amendt, P., Rosen, M. D., Guethlein, G., Bolton, P. R., and Wilks, S. C., Table-Top X-Ray Lasing Based on Optical-Ficld-Induced Ionization, Lawrence Lis urmore National Laboratory, Livermore, CA, UCRL-JC-110043 REP (1993). Prepared for 3rd Intcrnational Colloquium on X-Ray Lasers, Schliersee, Germany, May 18-22, 1992 (Inst. Phys. Conf. Ser. 125, 4).

Eimerl, D., Milam, D., and Yu, J., Large Benduidth FrifuencyConterted Nd:Glass Laider at $527 \mathrm{~mm}$ with $\Delta V / V=2 \%$, Lawrence Livermore National Laboratory, Livermore, CA, UCRL-JC-111297 (199.3). Prepared for Phy/s. Re't'. Le'th.

Erlandson, A. C., Gain Lniformity and Storagi' Efficioncy of a FlashlampPumpu'd Nd:Glass Multise'gme'nt Amplificr, Lawrence Livermore National Laboratory, Livermore, CA, UCRL-MI-112362 (1993). Prepared for Adzanced Program International Conferenci', I louston, TX, December 7-10, 1992 .

\section{$\mathbf{F}$}

Forest, J. A., Brooks, R. L., Schou, J., Stenum, B., Hunt, J. L., Sorensen, 11., Gurtler, P., Magnotta, F., and Mapoles, E. R., Comtinumm Emission from Irrudiafed Solid De'uterium, Lawrence Livermore National Laboratory, Livermore, CA, UCRI-JC-110969 (199.3). P'repared for Illiss. Reri. B.

Friedman, A., Introduction Second Merting of FtAC Pand 7 Inertial Fusion Energy, Lawrence Livermore National Laboratory, L.jvermore, CA, UCRL-MI-112373 (1993). Prepared for Fusion Linergy Adaisony Committe' Pancl > Me'ting, Berkeley, CA, December 7-8, 1992

Friedman, A., ITT Issules: Recirculating Induction Accelerator, Lawrence Livermore National Laboratory, Livermore, CA, UCRL. M1-113231 (1943).

Friedman, A., Heary lon Fusion (Ferribu, Lawrence Livermore National Laboratory, Livermore, CA, UCRL-MI-113232 (1993).

Friedman, A., Callahan, D. A., Langdon, A. B., Haber, I., Cirote, D. P'.,

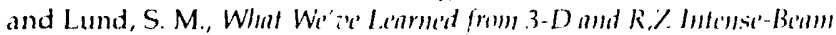
Simulutions Llsing the WARP' Code, Lawrence Livermore National Laboratory, Livermore, CA, UCRL.-JC-1!328.3 ABS (199.3). Prepared for International stmpusium on Hency lon Inertial Fission, Frascati, Italy, May 25-28, 1493.

Friedman, A., Cirote, D. P., Haber, I., Langdon, A. B., and Callahan,

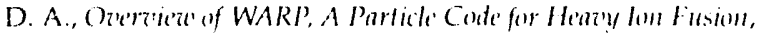
Lawrence Livermore National Laboratory, Livermore, (A, UCRI.MI-112246 (199.3). Prepared for CAP9.3 Pleasanton, CA, Fobruary 23-26, 1493,

G

Glendinning, S., Weber, S., Dixit, S., Da Silva, L., Bell, I., I Ienesian, M., Kania, D., Kilkenny, J., and Powell, H., Last'r-Dria'n Plantur Ravleigh-Tinylor Instubility Lxperiments, Lawrence Livermore National Laboratory, Livermore, (A, LCRL-JC-1119941 (1943). Prepared for Phys Re'is lett.
Glendinning, S. G., Weber, S. V., Kilkenny, J. D., Henesian, M. A., Dixit, S., Powell, H. T., Wallace, R., Knauer, J., and Verdon, C., Laser Driven Hydrodynamic Instability Experime'nts on Nor'n, Lawrence Livermore National Laboratory, Livermore, CA, UCRL-JC-106718 ABS, Rev. 2 (1993). Prepared for 1993 IEEE International Conference on Plasma Sicienc', Vancouver, British Columbia, Canada, June 7-9, 1993.

Glinsky, M. E., and Kruer, W. L.., Simple Model of Suprathermal Electron Transport, Lawrence Livermore National Laboratory, Livermore, CA, UCRL-JC-111436 SUM (1993). Prepared for Short Wazelength V: Physics uith Int'nse' Lase'y Pulse's, Second Topical Me'ting, San Diego, CA, March 29-31, 1993.

Greiner, G., Trent, M., Bernat, T. P., and Digrazia, It., Facility Sufety Procedure Building 298 Complex, Lawrence Livermore National Laboratory, Livermore, CA, UCRL-FSP-298 (1993).

Grote, D. P., Friedman, A., and Haber, I., Thre' Dime'nsional PIC Simulation of Heacy lon Fusion Beams: Recent Impronements to and Applications of WARP, Lawrence Livermore National Laboratory, Livermore, CA, UCRL-JC-112508 ABS (1993). Prepared for PAC 9.3, Washington, DC, May 17-20, 1993.

Grote, D. P., Friedman, A., and Yu, S., 3D Simulations of im Elictrustatic Quadrupule' Injector-Re'r. 1, Lawrence Livermore National Laboratory, Livermore, CA, UCRL-MI-111188, Rev. 1 (1993). Prepared for CAP93, Pleasanton, CA, February 23-26, 1993.

\section{$\mathbf{H}$}

Hammel, B. A., Keane, C. J., Lee, R. W., Kilkenny, J. D., Kania, D. R., and Pasha, R., X-Ray Spectroscopic Me'asurements of High De'nsitie's and Temperature's from lintirectly Drie'n ICF Capsuste's, Lawrence Livermure National Laboratory, Livermore, CA, UCRL-JC-111184 (199.3).

Prepared for l'hys. Re't' Le'th.

Itammel, B. A., Remington, B. A., Oades, K., Goldsack, T. J., Kilkenny, J. D., and Thomas, B., X-Ray/ Radiographic Me'asurements of Kelinin-Helmiloltz Groneth in X-Ray Drize'n Solid Density Materials, Lawrence Livermore Nation.al Laboratory, Livermore, CA, UCRLMI-112385 (1993). Prepared for 4th International Workshop on the' Plyysics of Compressible Tirrbulent Mixing, Cambridge, England, March 29-April 1, 1993

Henesian, M., Dixit, S., Menyuk, C., Wai, P., and Chen, C., Simulations of Spectral Broudening by Cross-Phose Modulation (XPM) will Chantic Laser Pulse's, Lawrence Livermore National Laboratory, Livermore, CA, UCRL-MI-112742 (1943). Prepared for 1993 SPIL Short-Pulse High-Intensity Lasers and Applications II, Los Angeles, CA, January $21-22,1993$.

Henning, C. D., Storm, E., and Correll, D., Notu LIprade Facility for lynition and Gain, Lawrence Livermore National Laboratory, Livermore, C $\therefore$, UCRL-LR-106874, Rev. I (1993).

Hewett, D. W., Chen, Y-J., and Yu, S. S. Simulation of the $1 \mathrm{mV}$ lon

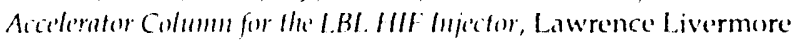
National Laboratory, Livermore, CA, UCRI -J (-11.3230) ABS (199.3). Prepared for 1993 IIFEE International Conferencie on Plasma Silince, Vancouver, British Columbia, Canada, June 7-4, 1993.

Hjorth, P. C., and Cilinsky, M. F., Helicity in Hamiltonian Dymamical Systems, Lawrence Livermore National Laboratory, Livermore, CA, UCRL-JC-112520 ABS (1493). Prepared for SIAM Comference om Applications of Dymumical Systemls, Snowbird UT, () ctober, 1992.

Ho, D., and (randall, K., Sextuphle Correction of Second-() reder

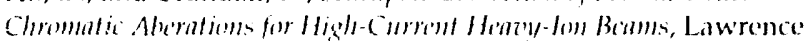
Livermore National Laboratory, Livermore, (A, UCRL-JC-110212 ABS, Rev. 1 (1993). I'repared for 1993 Particle Accle'rutor Comferenti', Washington, D.C., May 17-20), 1993. 
Ho, D. D-M., Lir,dl, J. D., and Tabak, M., Radiation Conzerter Physics and a Methodolgy for Obtaining the Upper Limit for Gain for Heary-Lon Fusion, Lawrence Livermore National Laboratory, Livernore, CA, UCRL-JC111176 ABS, Rev: 1 (1993). Prepared for International Symposium on Henty lon Imertinl Fusion, Frascati, Italy, May 25-28, 1993.

Ho, D. D-M., and Tabak, M., Target Physics for Hollty-lon Fusion, Lawrence Livermore National Laboratory, Livermore, CA, UCRL-JC113454 ABS (1993). Prepared for 1993.3 Topical Conference: the Physics of Radiotizely Drizen ICF Targ'ts, Monterey CA, April 26-29, 1993.

Hogan, W. J., Status of the Fusion Program for Cirilim Applications, Lawrence Livermore National Laboratory, Livermore, CA, UCRLMI-113161 (1993).

Hogan, W. J., Bangerter, R., and Kulcinski, C. L., Energy from linertial Fusion, Lawrence Livermore National Laboratory, Livermore, $C A$, UCRL-JC-111596 (1993). Prepared for Plyysics Toddry.

\section{$\mathbf{K}$}

Kilkenny, J. D., Baldis, H. A., Darrow, D. B., Corok, R. C., Cable, M. D., Dittrich, T., Ellis, R. J., Gilendinning, S. C., and Haan, S. W., Re'cent Experimental Results on Noru, Lawrence Livermore National Laboratory, Livermore, CA, UCRL-JC-113201 ABS (1993). Prepared for 1993 ItEL: Intermational Conferefice' on Plusma Seitule', Vancouver, Britisi. Columbia, Canada, June 7-9, 199.3.

Kilkenny, J. D., Munro, D., Remington, B. A., Clendinning, S. Ci., Haan, S., Weber, S., Wallace, R., P'owell, H., and Dixit, S., Exprerime'ntal Determination of the Hydrodynamic Hostability Corouth Rote's in Indired and Dired Drize ICF, Lawrence Livermore National Laboratory, Livermore, CA, UCRL-JC-1](1277 (1993). Prepared for 141/1 IAEA

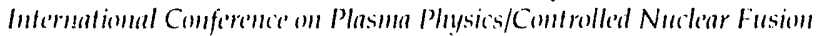
Re'seirch, Wur/burg, Germany, September 31-October 7, 1992.

Kilkenny, J. D., Munro, D. H., Remington, B. A., Glendinning. S. C., Haan, S. W., Weber, S., Wallace, R. J., Knauer, J. P., and Verdon, C. P., Experime'ntal Determination of the Hydrotymamic Instability Croneth Rate's in Indired and Dired Drize ICF, Lawrence Livermore National Laboralory, Livermore, (A, UCRL-JC-11(1277 ABS, Rev. 1 (1993).

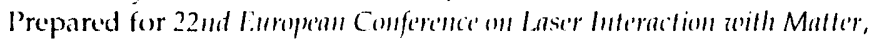
Paris, France, May 11)-14, 1493.

Kirbie, H. C., Cravey, W. R., Hawkins, S. A., Newton, M. A., and

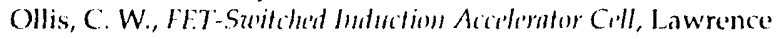
Livermore Naticnal Laboratory, Livermore, CA, UC RL-JC-112510 ABS (1993). Prepared for 1993 IFEF International Conference' an Plasma Sidence, Vancouver, British Columbia, Canada, June 7-9, 1993.

Klem, D., Darrow, C., Perry, M., and Lane, S., Alsorption and $x$ Rayls from Short Pulst's, Lawrence Livermore National Laboratory, Livermore, CA, UCRL-MI-112728 (1993). Prepared for SPIt: Optical Enginerering Lase' '9.3 Comference', Los Angeles, CA, January 16-23, 1943.

Koch, J. A., Batson, I?. J., Matthews, D. L., MacGowan, B. J., Da Silva, L. B., Mrowka, S., Nilsen, J., and Underwood, J. II., High-Resollution Spectroscopy als an X-Ray Iaser Plasma Dingnestic, Lawrence Livermore National Laboratory, Livermore, CA, UC RL-JC-11.3287 ABS (1993).

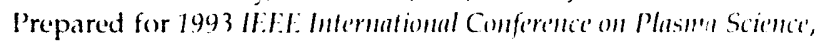
Vancouver, British Columbia, Canada, June 7-4, 1993.

Koch, J. A., Macciowat, B. J., Mrowka, S., Matthews, D. L., Da Silva, L. B., Underwood, J. H., and Batson, P. J., Sele'nium X-Ray Lase'r Lim' Profile Me'nstereme'nts, Lawrence Livermore National Laboratory, Livermore, (A, UCRL-JC-110974 REP. Prepared for 3rt lithernational Colloquium on X-Raly Lalse's, Schliersee, Ciermany, May 18-22, 1992 (Inst. l'hys. Conf. Ser. 125).

Kozlowski, M. R., Thomas, I. M., Rainer, F, and Campbell, J. H.,

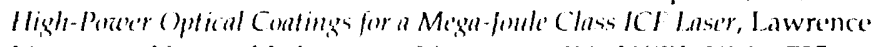
Livermore National Laboratory, Livermore, (A, UCRL-JC-1114735 (1993). Prepared for linternational Sympusium on (1ptical Desion" Systems, Berlin, Germany, September 14-18, 1492.
Kruer, W. L., Lasi'r Plasma lssile's for Megajould ICF Targcts, Lawrence Livermore National Laboratory, Livermore, CA, UCRL-MI-106368, Rev. 1 (1993). Prepared for LLLVI./Commonzeralth of Independint States Workshop, Livermore, CA, February 8, 1993.

Kruer, W. L., and Wilks, s Z., Llltra-Intensi' Laser Plasma Interactimes, Lawrence Livermore National Laboratory, Livermore, CA, UCRLMI-113162 (1993). Prepared for I.I.NI./Commonuerealth of Ind'pu'nde'nt State's Workshop, Livermore, CA, February $8,1943$.

Kway, W. L., Payne, S. A., Smith, L.. K., Tassano, J. B., Deloach, L. D., Krupke, W. F., and Chai, B. H. T., Crystal Grouth and Characterization of Fltommatite and Almologs, Lawrence Livermore National Laboratory, Livermore, CA, UCRL-MI-113448 (1993). Prepared for Gordon Re'senerh Comference' on Crystal Croneth, Oxnard CA, March 15-19, 1993.

\section{L}

Landen, (O. L., High Resolution Time- and 2-Dimensiomal Spuce-Re'solzed $X$-Ray lmaging of Plasimas al Norn, Lawrence Livermore National Laboratory, Livermore, CA, UCRL-JC-1(1)9094 (1993). Prepared for

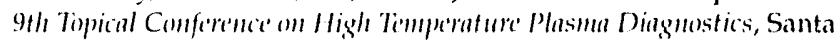
Fe, NM, March 15-19, 1993.

Landen, (). L.., Cilendinning, S. (;., and Munro, D. H., Time'-Re'solterd (Ipacily of Iudirectly-Drizen Fhorusilicame Foils, Lawrence Livermore National Laboratory, Livermore, CA, UCRL-JC-11346I ABS (1943). Prepared for 1993 Tippical Comference: the Physics of Radiationdy Drize'll KC Tirgets, Monterey (A, April 26-29, 1993.

Landen, (). L.., Cilendinning, S. G., Ress, D. B., Remington B. A., Hammel, B. A. Turner, R. E., I lauer, A. A., Hsing, W. W., and

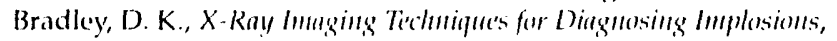
Lawrence Livermore National Laboratory, Livermore, CA, UCRLMI-11.3052 (199.3).

Landen O. L., Levedahl, K., and Dittrich, T., X-Ray Imaging of PusherFicl Interface in ICF Implesioms, Lawrence Livermore National Laboratory, Livermore, CA, UCRL-JC-113460) ABS (1993). Prepared

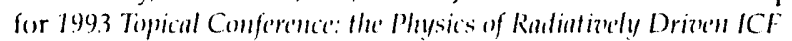
Targets, Monterey, CA, April 26-29, 1943.

Lane, S. M., Cauble, R., Cilendinning, S. C., and Da Silva, L. B., Simple Model for the Determination of Lase'r Ablation Pressure from Shock Transit Timu Me'asureme'nts, Lawrence Livermore National Laboratory, Livermore, CA, UCRL-JC 11.34.30 ABS (199.3). Prepared for $199.3 \mathrm{IELE}$ International Confere'nce' on Plasma Science', Vancouver, British Columbia, Canada, June 7-9, 1993.

Lawsen, J. K., Dixit, S. N., Manes, K. R., Henesian, M. A., Eimerl, D., Morgan, A. J., P'owell, II. T., Thumas, I. M., and Trenholme, J. B., Planse'

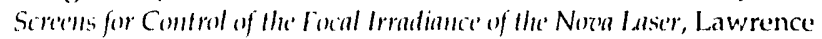
Livermore National laboratory, Livermore, (A, UCRL-MI-111445 (1993). Prepared for 1993 SPII: Short-Pulse High-Intensity Lasers and Applications II, Los Angeles, CA, January 21-22, 1993.

Lindl, J. D., Progress and Challenge's in ICF Physics, Lawrence Livermore National Laboratory, Livermore, CA, UCRL-MI-1130170

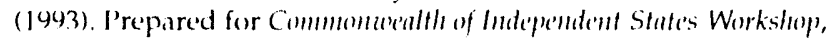
Livermore, CA, February 9, 1943.

Lindl, J. D., Progerses and Prospects for Indired Drize $I C l$, Lawrence Livermore National Laboratory, Livermore, (A, UCRL-JC-111)279 (1993). P'repared for 1+th IALA Internatiomal Conforinte on Plasma Physics/Controlled Nurlear lission Rescench, Wurzburg, Germany, September 30-October 7, 1992.

London R. A., Trebeen, J. E., and Jacobsen, C. J., Role of X-Ray Indinced Dammag, in Biological Microimagring, Lawrence Livermore National Laboratory, Livermore, ( A, UCRL-JC-1(19590 REP (1993). P'repared

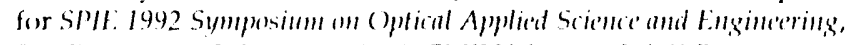
San Diego, CA, July 19-24, 1992 (SIPIE Vol. 1741 Solt $X$ Ray Morosiony ly92, Beilingham, WA). 


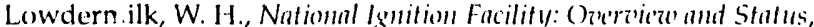
Lawrence Livermore National Laboratory, Livermore, CA, UCRLMI-113080 (1993). Prepared for LI.NL/Commomterealth of Independe'nt State's Workshop, Livermore, CA, February 8,1993

\section{$\mathbf{M}$}

MacGowan, B. J., Prohlems with X-Ray (ppics at LIINI., Lawrence Livermore National Laboratory, Livermore, CA, UCRL-MI-1130174 (1993). Prepared for Commomenealth of Indepe'mil'nt Stale's Workshop, Livermore, CA, February 9, 1993.

MacCowan, B. J., X-Rny Damage fo Multilayers, Lawrence Livermore National Laboratory, Livernore, CA, L¿RL-MI-113084 (1993). Prepared for Soft X-Ruys in the' 21st C'ntury, Provo, UT, Fobruary 1) 13,1993

MacGowan, B. J., Soft X-Ray Laser Re'singh-2, Lawrence Livermore National Laboratory, Livermore, CA, UCRL-MI-113222 (1993). Prepared for LI.NL/Commminteralth of Independent State's Workshop, Livermore, CA, Febrwary $8,1993$.

Mapoles, E. R., Beta L.ayering Lpedat', Lawrence Livermore National Laboratory, Livermore, CA, UC.RL-MI-11.3226 (1493). Prepared for I.I.NI./Commmomerealth of Independe'nt State's Workshoph, Livermore, CA, February 8,1943

Matthews, D., Soft X-Rmy Lasi'r Re'sermeh, Lawrence l.jvermore National Laborntory, Livermore, CA, UCRL-MI-1131775(1943).

Murray, J. R., Campbell, J. I., and Frank, D. N., Betmele' I.ase'?

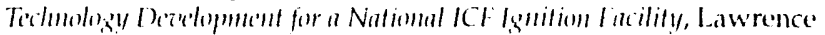
Livermore National Laboratory, Livermore, CA, LCRL_-JC-1]345] ABS

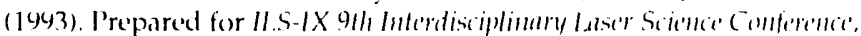
(OSA Me'ting, Toronto, (Ontario, Comada, ()ctober 3-8, 194,3,

\section{$\mathbf{N}$}

Newton, M. A., HII lingintering af LI.NI., Lawrence Livermore National Laboratory, Livermore, (A, UCRL..MI-11.3586 (1493).

Newton, M. A., Cravey, W. R., Ollis, C. W., Kirbie, H. C., and

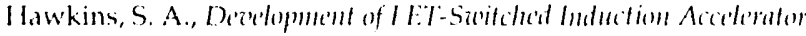
Cells for Heray-lon Recirculators, Lawrence Livermore National Laboratory, Livermore, CA, UCRL-JC-112375 ABS (1993). P'repared for $P A C$ 93, Washington, DC, May 17-20, 1493.

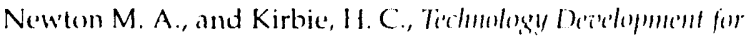
Recirculuting Heary-Len Accelerutors, Lawrence Livermore National Laboratory, Livermore, CA, UCRL-KC-11.3282 ABS (1943). Prepared

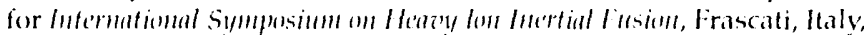
May 25-28, 1993.

\section{O}

(Ollis, C. W., Cravey, W. R., Hawkins, S. A., Kirbie, H. C., and Newton,

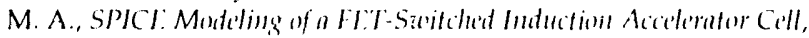
Lawrence Livermore National Laboratory, Livermore, CA, UCRI-KC112511 ABS (1993). Prepared for 9th IIEE Intermational Pulsed Porter Conference, Albuguerque, NM, June 21-23, 1993.

\section{$\mathbf{P}$}

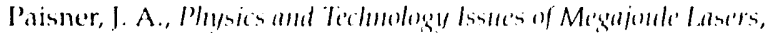
Lawrence Livermore National Laboratory, Livermore, CA, UCRL.

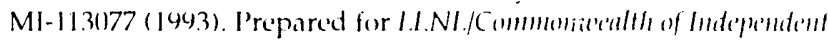
States Workshop, livermore, CA, February $8,1993$.
Payne, A. N., System Modeling for the Longitudinal Beam Dymamics Comtrol Problem in Heney lon Indaction Accelerators, Lawrence Livermore National Laboratory, Livermore, CA, UCRL-JC-1/2378 ABS (1993). Prepared for PAC 93, Washingt(on, DC: May 17-20, 1993.

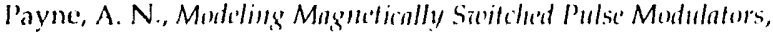
Lawrence Livermore National Laboratory, Livernore, CA, UCRL-JC-

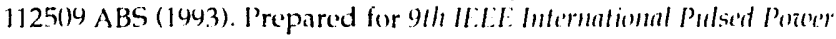
Comference, Albuquercue, NM, June 21-23, 1993.

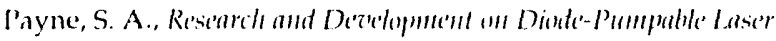
Crystals, Lawrence Livermore National Laboratory, Livermore, CA, UCRL-JC-11.32.38 ABS (1993). Prepared for Americm Comference on Crystal Grouthth. Baltimore MD, August 1-6, 144.3.

Payne, S. A., Krupke, W. F, Kway, W. L., Smith, L. K., Detoach, L. D., Tassano, J. B., and ('hai, B. I1. T., Propertie's of the Yb-Fluorapalite lalse'r and Related Structural Amulegss, Lawrence Livermore National Laboratory, Livermore, CA, UCRL-MI-111372 (194.3). P'repared for

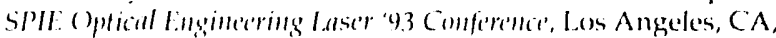
January $16-23,1993$.

I'ayneS. A., Smith, L. K., Tassano, J. B., Kway, W. L., Deloach, L. D.,

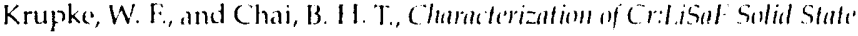
Lasir Properties, Lawrence livermore National Laboratory, Livermore, CA, UCRL-MI-11150n (1993). Prepared for SPlt: (optical linginecring

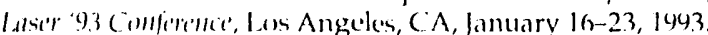

Penningtom, D. M., and I larris, C. B., Dymamies of Surfane Thermal

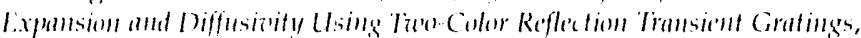
Lawrence Livermore Nalional Lahoratory, l.jvermore, CA, UCRL-

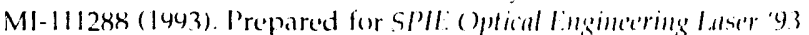
Comferenci, los Angeles, (A, lanuary 16-23, 1493.

Pennington, D). M., Henesian, M. A., Thompson, ( . F., Powell, I1. T,

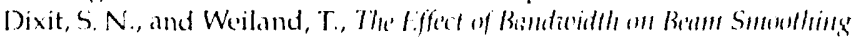

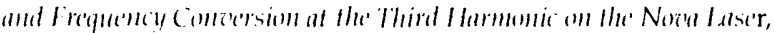

Lawrence Livernore National laboratory, Livermore, CA, UC RL-MI-

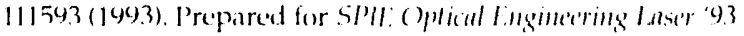
Comfermot, Lom Angedes, (A, January 16-23, 1493.

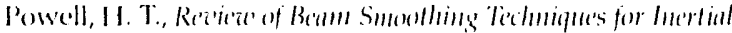
Comfincment lusiont, Lawrence Livermore National Laboratory, Livermore, (A, UCRL-MI-112643 (1993). Prepared for SPll: ()plical

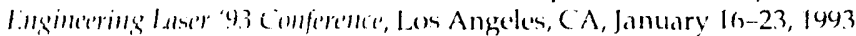

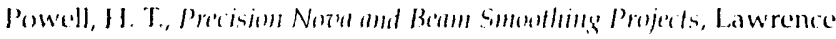
Livermore National Laboratory, L.ivermore, CA, UC RL.-MI-1131182

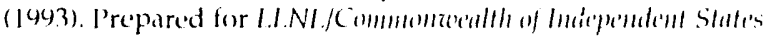
Workshep, Lidermore, CA, Fobruary 8, 1943.

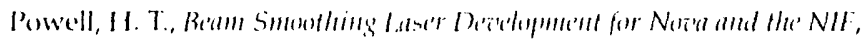
Lawrence Livermore Notional Laboratory, Livermore, CA, LCRL-

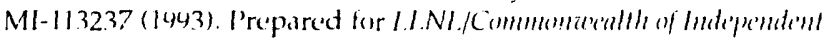
Stale's Workshop, l.jvermore, (A, February $x, 1993$.

Procassini, R. J., and Knotl, D. A., Kintically Motizuted Boumdtury

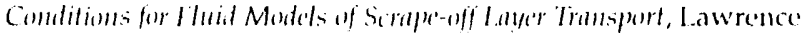
Livermore National Laboratory, Livermore, CA, UCRL-JC-110317 (1493). Prepared for /. Nici. Mat., 2023D, 196-198, 363-368 (14921.

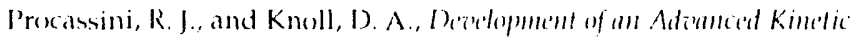

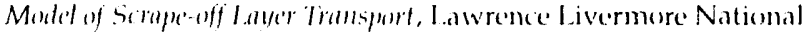
Laboratory, Livermore, (A) UCRL-MI-1125116 (1943). Prepared for

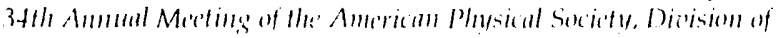
Plisima Pluysics, Seattle WA, November 16-20, 1992.

\section{$\mathbf{R}$}

Rainer, F., Atherton, I. J., and De Yoreo, J. J., Laser Dammege fir

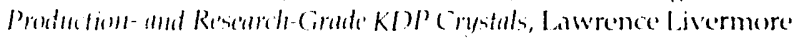
National Laboratory, Livermore, CA, UCRI-KC-11/46.3(1493). P'repared

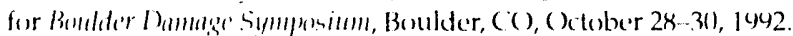


Ratowsky, R. P., Craxton, R. S., Walling, R. S., London, R. A., Feit, M., Shimkaveg, (i., and Carter, M. R., Thre' Dime'nsional Time'-De'pende'nt Hydrodymamic and Propagatiun Modeling of X-Ray Laser Emissim, Lawrence Livermore National Laboratory, Livermore, CA, UCRL-JC110000 REP' (1993). Prepared for 3rt lintermational Collonpumm on $X$ Ray Lase's, Schliersee, Germany, May 18-22, 1992 (Inst. Phys. Conference. Ser. 125, 6).

Remington, B. A., Weber, S. V., Wailace, R. J., Kilkenny, J. D., and Haan, S. W., Mullimode and 3D Raylegigh-Tinglor lixperiments on Noru, Lawrence Livermore National Laboratory, Livermore, CA, UCRL-JC111171 ABS, Rev. 1. (1993). Prepared for thl linternationul Workshopt on the' Physits of Compressible Tirblukent Mixing, Cambridge, England, March 29-April 1, 1993.

Remington, B. A., Weber, S. V., (ilendinning, S. G., Kilkenny, J. 1)., Haan, S. W., and Wallace, R. J., Laser Drisen Hydrotymmic Instability Exprime'nts, Lawrence Livermore National Laboratory, L.jvermore, CA, UCRL-JC-111286 (1993). P'repared for 34 th Ammenl Meteting of the Americun Physical Sociefy, Dizisionn of Plasimu Physicts, Seattle WA, November 16-2(1), 1992.

Remington, B. A., Weber, S. V., Wallace, R. J., Kilkenny, J. D., and

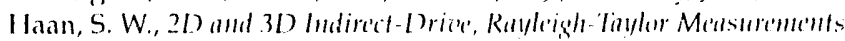
on Nor'a, lawrence Livermore National laboratory, Livermore, (A UCRL-JC-113462 ABS (1993). Prepared for 199.3 Topical Conformer:

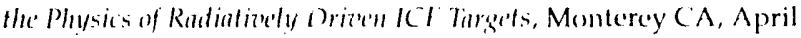
$26-29,1993$.

Remington, B. A., Weber, S. V., Wallace, R. J., Kilkenny, J. D., and

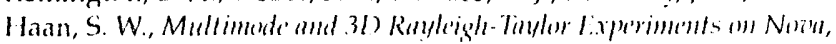
Lawrence Livermore National Laboratory, Livermore, ( A, UCRL. MI-111171 (1993). Prepared for th hitternational Workshol on the Physics of Comprossible Tirrbulent Mixing, Cambridge, lingland, March 29-April 1, 1493.

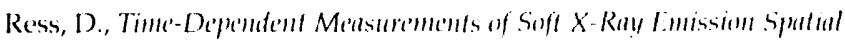
Profille's, Lawrence Livermore National Laboratory, Livermore, CA,

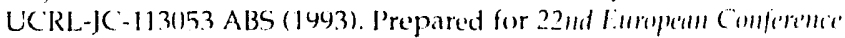
on lasiser Interattion with Matter, Paris, France, May 11-14, 1993.

Ress, D., Trebes, J. E., Procrassini, R., Mrowka, S., Da Silva, 1. L , and London, R. A., Soft X-Rouy Moire Deflectemeter for Characterizalions of High-Density Plasima, Lawrence Livermore National laboratory, Livermore, CA, UCRL-MI-112.386 (199.3). Prepared for Soff X-Rays in the' 21st Coulury, Provo, UT, February 1(1)-13, 1993.

Rhodes, M. A., and Taylor, J. B., Pulse' Poreer Re'puirements for large'

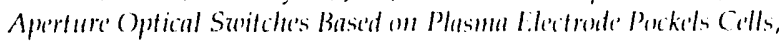
Lawrence Livermore National Laboratory, Livermore, (A, UCRI. JC -

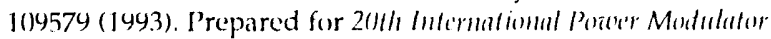
Symposium, Myrtle Beach, SC, June 23-25, 1942.

Rotter, M. D., Erlandson, A. C., and McCracken, R. W., Analytic

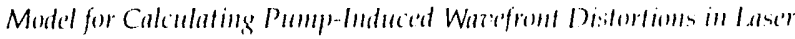
Slabs, Lawrence Livermore National Laboratory, Livermore, $(\mathrm{A}$. UCRL-MI-1111933 (1993). P'repared for Adidnci' P'rogram Intirmatimul Conference' on Lasers '92, Houston, TX, December 7-10, 1992.

\section{S}

Sanche\%, J. J., l.iguid lanyers, Lawrence Livermore National Laburatury, Livermore, (A, LC RL-MI-1/3227 (1493). P'repared for

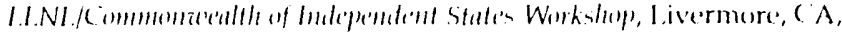
Fobruary $x, 1993$.

Sharp, W. M., Barnard, J. J., (irote, I). I., and Yu, S. S., Rncedope Model of Beum Trumspert in II.S1., L awrence Livermore National I aboralery, Livermore, CA, UCRL -MI-112244 (1943). I'repared for CAP9?

Ileasantom, (A, february 23-26, I463.
Sharp, W. M., Callahan, D. A., Fessenden, T. J., and Langdon, A. B., Correction of Longitudinal Firors in Accelerators for Heary-Lom Fusion, Lawrence Livermore National Laboratory, Livermore, CA, UCRL-JC112.377 ABS (199.3). Prepared for PAC 93, Washingtom, DC, May 17-201, 1943.

Sharp, W. M., Rangarajan, C; and Sesster, A. M., Mulli-Morde DiscreteConily Simulation of a Standing-Wan' frere-filectron l.aser, Lawrence Livermore National Liboratory, livermore, CA, UCRI.-JC-112662 ABS (199.3). Prepared for 199.3 Internutional Symmpesium on (Optical Applind Scivince and Engindering, San Diego, (A), July 11-16, 1993.

Smith, L. K., Payne, S. A., Kway, W. L., Deloach, L. D., Krupke, W. L., and Chai, B. H. T., Delosednation of laser Efficiencies for Yb- Doped Apatile Stracture Hests, Lawrence Livermore National Laboratory, Livermore, CA, UCRI.-MI-111850) (1৬9.3). Prepared for Adrumced Sollid Stath LAsi's, New Orleans, LA, Februany 1-3, 1943.

Staggs, M. C., Balooch, M., Kozlowski, M. R., and Siekhaus, W. J.,

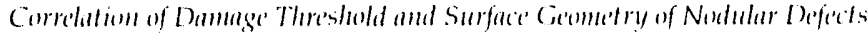
in HR Coatings as Determinued by In-Situ Alomic Rorce Microscopy, l.awrence Livermore National Laboratory, Livermore, CA, UCRL-JC111464 (1993). I'repared for Boulder Dammage Symmesium, Benlder, (o), October $28-30), 1942$.

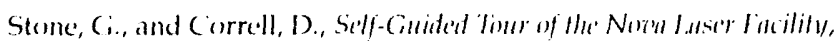
Lawrence livermore National Laboratory, Livermore, CA, UC'RI$T 13-11(1) \times 73(1993)$

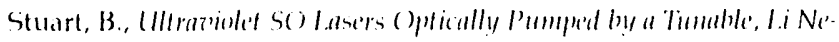

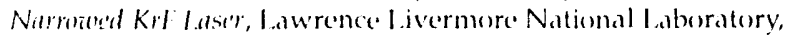
Livermore, (A, UCRI-LR-III1935 (1943)

\section{T}

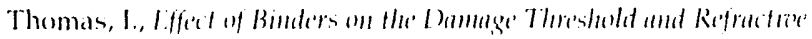

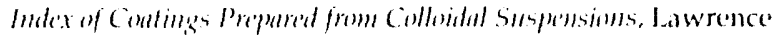
L.ivermore National Iaboratory, I ivermore, (A, UCRL-JC-1115211 (1993). I'repared for Bondider Dammi, Sympusilum, Boulder, (C), (october $28-30,1492$.

Trebes, J., Spulial Colleremle of Suft X Ray I.tusers, Lawrence I.ivermore National Laboratury, Livermore, C A, UCRL-J - -111342 RER? I'repared

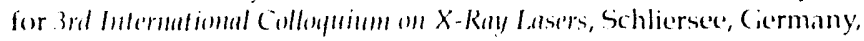
May 18-22, 1992 (Inst. Ihys. Conference. Ser. 125).

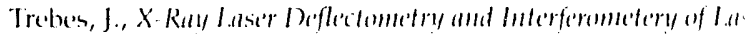
Prodeced Plasmess, Lowrence Livermore National Laborators: Livermure, (A, UCRI.-MI-112297 (1993). Prepared for .34th Ammual

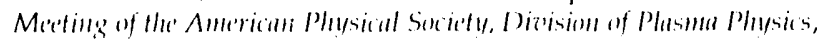
Seatlle WA, November 16-201) 1992.

Trebes, J., Balhorn, R., Eder, D., Da Silva, L., Barbee, T., Kench, J., Lehr, D., London, R., and Macciowan, B., Applications of X-Rayl Landers II, Lawrence livermore National Laboratory, livermore, CA, UC RI.-JC-

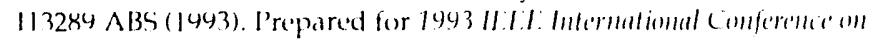

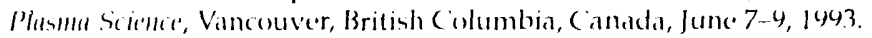

Trebes, J., Balhorn, R., Eder, D., Da Silva, L.., Barbee, T., Kond, J., I dehr,

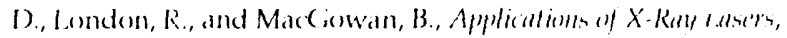
Lawrence livermore National Laboratery, Livermore, ( $A$, UCRI -JC -

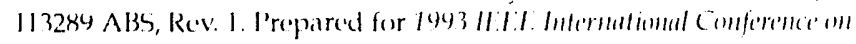
Plasma Scintere, Vancouver, British Columbia, Conada, June 7 -9, J493.

\section{V}

Vann, C., Murray, J., Rushford, M., Mewre, K., J:llis, R., and Seppala,

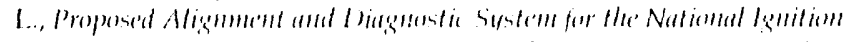

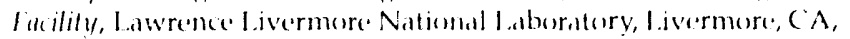

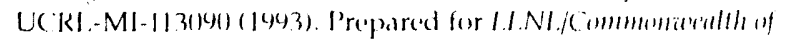

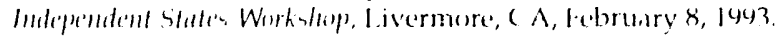


Villeneuve, D. M., Lafontaine, B., Enright, G. D., Dunn, J., Baldis, H. A., Rosen, M. D., Young, P. E., and Matthews, D. L., Temperature' Determination in X-Ray Laser Plasmas by Thompson Scattering, Lawrence Livermore National Laboratory, Livermore, CA, UCRL-JC109721 (1993). Prepared for Fifth Topical Meeting of the OSA on ShortWavelength Colerent Radiation: Generation and Applications, Monterey, C A, April 8-10, 1991.

\section{W}

Weber, S. V., Remington, B. A., Nash, J. K., Wilson, B. G., and Haan, S W., Opacity Effects in Norn Indirect Drive Rayleigh-Taylor Experiments, Lawrence Livermore National Laboratory, Livermore, CA, UCRL-JC113455 ABS (1993). Prepared for 1993 Topical Conference: the Plyysics of Radiatize'ly Drizen ICF Targets, Monterey CA, April 26-29, 1993.

Wegner, P., Measur'me'nts and Modeling of Laser Irradiance in the HighPower Third-Harmonic Notn Focus Volume B: Datn Book, Lawrence Livermore National Laboratory, Livermore, CA, UCRL-ID-110480, Vol. 2 (1993)

Wilcox, R., Browning, D., Van Wonterghem, B., Behrendt, W., and Speck, D., Fusion Laser Oscillator and Pulse-Forming System Lising Integrated Optics, Lawrence Livermore National Laboratory, Livermore, CA, UCRL-MI-111393 (1993). Prepared for SPIE Optical Engine'ring Laser '9.3 Conference', Los Angeles, CA, January 16-23, 1993.
Wilcox, R. B., Behrendt, W., Van Wonterghem, B. M., Speck, D. R., and Browning, D. F., Fusion Laser Oscillator and Pulse-Forming System Using Integrated Optics, Lawrence Livermore National Laboratory, Livermore, CA, UCRL-JC-111393 (1993). Prepared for SPIE Optical Engineering Laser '93 Conference', Los Angeles, CA, January 16-23, 1993.

Wilks, S., Mori, W., and Kruer, W., Odd Harmonic Ge'neration of UltraIntense Laser Pulses Reflected from an Overdense Plasma, Lawrence Livermore National Laboratory, Livermore, CA, UCRL-MI-112681 (1993). Prepared for SPIE Optical Engineering Laser '9.3 Conference', Los Angeles, CA, January 16-23, 1993.

Wilks, S. C., Langdon, A. B., Mori, W., and Kruer, W., Simulations of Ultra-Intense' Lase't Plasma Interactions, Lawrence Livermore National Laboratory, Livermore, CA, UCRL-MI-112253, Rev. 1 (1993).

Prepared for SPIE Optical Engine'ring Lase'r '9.3 Conference, Los Angeles, CA, January 16-23, 1993. 

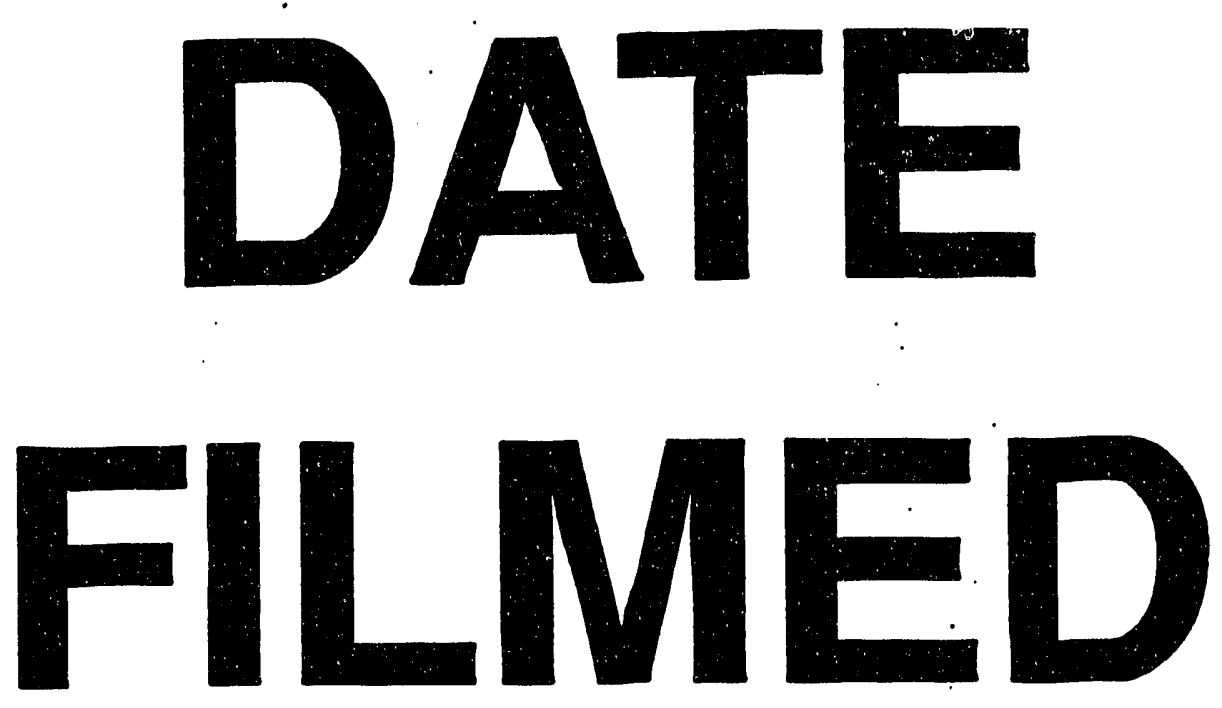

$11 / 4 / 93$
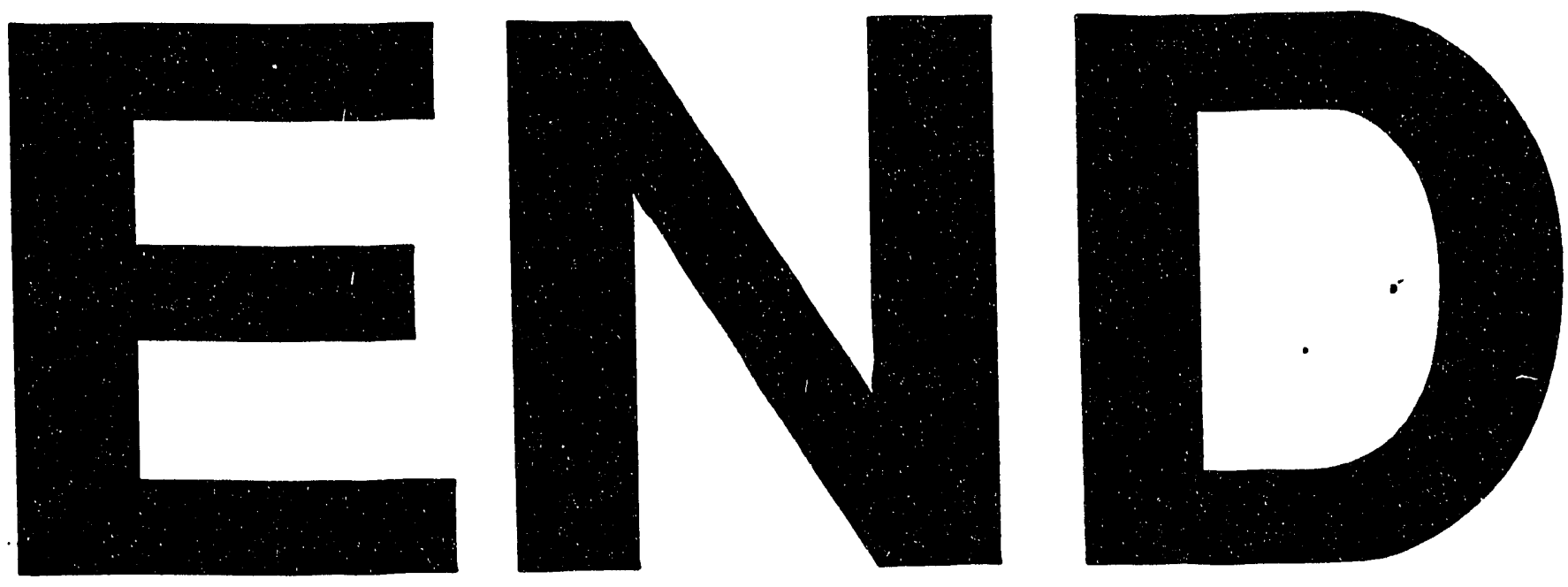
